\title{
A Workload-Driven Framework for NoSQL Data Modeling and Partitioning
}

by

\begin{abstract}
Ali Davoudian
A proposal submitted to

the Faculty of Graduate and Postdoctoral Affairs

in partial fulfilment of the requirements for the degree of

Doctor of Philosophy in Computer Science
\end{abstract}

Ottawa-Carleton Institute for Computer Science (OCICS)

School of Computer Science

Carleton University

Ottawa, Ontario, Canada

April 2021

Copyright (C)

2021 - Ali Davoudian 


\section{Abstract}

Due to the scalability problems in traditional relational database systems, a variety of NoSQL stores have emerged over the last decade to deal with big data. The lack of standard processes for designing and partitioning NoSQL datasets, as two non-orthogonal principles of distributed database systems, has led to the proposal of several recent methods. On the one hand, the existing design methods provide various conceptual modeling notations and mainly target a particular NoSQL data model that cause extra effort for designers when switching from one data model to another. Also, by providing just a set of guidelines and heuristics for the design process, many methods have to be applied manually which is an error-prone and time-consuming process. To deal with these limitations, we present a novel method for designing key-value, wide-column, and document NoSQL database schemas from the same conceptual model. It first generates a generic NoSQL logical schema from the conceptual model and query workload of the system. Then it converts the generic schema to the schemas of targeted NoSQL data models regarding their important features and design trade-offs between the read query performance and storage overhead or consistency maintenance.

On the other hand, the existing graph partitioning strategies are mostly workloadagnostic, as they presume the same probability of traversing edges or visiting vertices, which does not always hold with different query workloads. In addition, they are mostly graph topology-agnostic, as they do not differentiate between high-degree 
and low-degree vertices. Furthermore, many existing workload-aware strategies are unable to adapt to dynamic workloads. To address these limitations, we present a novel workload-adaptive and topology-driven approach named Helios, that aims to achieve low-latency and high-throughput online graph queries. In order to assess the impact of Helios on a graph store and to show how easily the approach can be plugged on top of the system, we exploit it in a distributed graph-based RDF store. The query engine of the store exploits Helios to reduce inter-node communication for future queries and balances the computational load across a cluster of nodes. 


\section{Acknowledgments}

First and foremost, I am immeasurably indebted to my supervisor Prof. Mengchi Liu, for his fundamental role in my doctoral research. Prof. Liu provided me with every bit of patient guidance, encouragement, and advice that I needed over the course of my Ph.D. journey. He gave me the freedom to do whatever I wanted, whilst continued to actively care about my work and promptly respond to my questions and inquiries. In addition to our scientific collaboration, I immensely value the personal rapport that Prof. Liu and I have forged over the past seven years. I am quite sure it is impossible to find a better supervisor. I would like to express my gratitude to all the staff members (both teaching and non-teaching) in the Ottawa-Carleton Institute for Computer Science, for their constant presence and the positive learning atmosphere without which this thesis would not exist. I would also like to express my heartfelt thanks to the anonymous reviewers of my publications for the donation of their expertise and effort in providing valuable comments and suggestions. 


\section{Table of Contents}

Abstract $\quad$ ii

Acknowledgments $\quad$ iv

Table of Contents $\quad$ v

List of Tables $\quad$ ix

List of Figures $\quad$ x

Nomenclature xiii

1 Introduction 1

1.1 Why NoSQL? .......................... 1

1.2 NoSQL database schema design problems . . . . . . . . . . 6

1.3 NoSQL graph partitioning problems . . . . . . . . . . . 8

1.4 Thesis aims and objectives . . . . . . . . . . . . . 9

1.5 Research methodology ................... 11

1.6 Thesis contributions ..................... 14

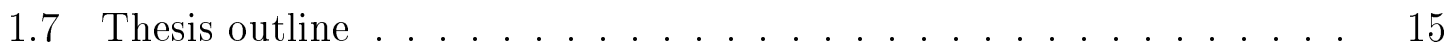

2 Literature Review $\quad 17$

2.1 NoSQL data modeling . . . . . . . . . . . . . 17 
$2.1 .1 \quad$ Key-value stores . . . . . . . . . . . . . . . . . . . 18

2.1 .2 Wide-column stores . . . . . . . . . . . . . . . . . 20

2.1 .3 Document stores . . . . . . . . . . . . . . . . . . 21

2.1.4 Graph stores . . . . . . . . . . . . . . . . 24

2.2 NoSQL data partitioning . . . . . . . . . . . . . . . . . 25

2.2.1 Key-oriented static partitioning $\ldots \ldots \ldots \ldots 27$

2.2.2 Key-oriented workload-aware partitioning . . . . . . . . 29

2.2 .3 Traversal-oriented partitioning . . . . . . . . . . . . 30

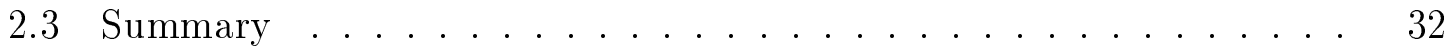

3 Partitioning of Aggregate-Oriented Databases 33

3.1 Concepts and formalization $\ldots \ldots \ldots \ldots \ldots \ldots$

3.1 .1 Running example . . . . . . . . . . . . . . . . 34

3.1 .2 KV schema formalization . . . . . . . . . . . . . . 35

3.1.3 Wide-column schema formalization . . . . . . . . . . 37

3.1.4 Document schema formalization . . . . . . . . . . . . 39

3.2 Related works . . . . . . . . . . . . . . . . . 40

3.3 Solution description . . . . . . . . . . . . . . . . . . . 42

3.3 .1 Conceptual schema . . . . . . . . . . . . . . . . . . . 43

3.3 .2 Query modeling . . . . . . . . . . . . . . . . 43

3.3 .3 Query path graph . . . . . . . . . . . . . . 45

3.3 .4 QPG specifications . . . . . . . . . . . . . 46

3.3.5 Mapping QPG to KV schemas . . . . . . . . . . . . 46

3.3.6 Mapping QPG to CF schemas . . . . . . . . . . . . 51

3.3.7 Mapping QPG to document collection schemas . . . . . . . 53

3.3 .8 Computational complexity . . . . . . . . . . . . . . 59

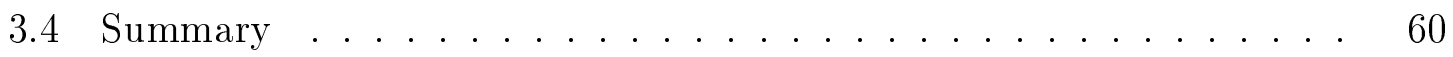


4 Partitioning of Graph Databases $\quad 61$

4.1 Related work ....................... 62

4.2 WASP framework ....................... 64

4.2.1 Data and query model ............... 64

4.2.2 Workload characteristics ............. 66

4.2 .3 Vertex reassignment ................ 67

4.2.4 Verifying WASP on an RDF store . . . . . . . . . . . 74

4.2.5 Memory and time complexities ........... 78

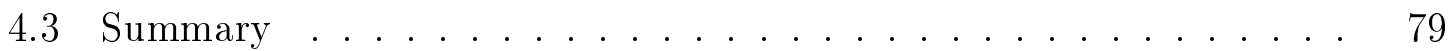

5 Empirical Evaluations $\quad 80$

5.1 Key-Oriented partitioner evaluation . . . . . . . . . . 80

5.1.1 QPG expressiveness ................ 81

5.1.2 Quality comparison ................ 82

5.2 Traversal-oriented partitioner evaluation . . . . . . . . . . . 94

5.2.1 Graph datasets and workloads . . . . . . . . . . . . 97

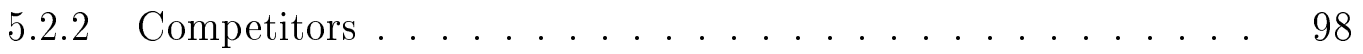

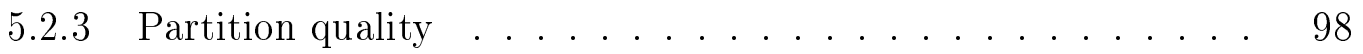

5.2 .4 Query performance ................ 101

5.2.5 Reassignment threshold sensitivity analysis . . . . . . . . . 102

5.2.6 Edge $\log$ size sensitivity analysis . . . . . . . . . . . . 104

5.2.7 Splitting threshold sensitivity analysis . . . . . . . . . 105

5.2 .8 Workload evolution . . . . . . . . . . . 106

5.2 .9 Data scalability ................... 106

5.3 Summary ............................ 107

6 Conclusion and Future Work $\quad 109$ 
List of References 


\section{List of Tables}

2.1 Various graph structures .................... 25

2.2 Comparison of the NoSQL data models . . . . . . . . . . 26

3.1 Database schema design processes for aggregate-oriented NoSQL stores 41

3.2 (a) specification of QPG nodes; (b) specification of QPG edges; (c) specification of QPG queries ............... 47

3.3 A list of functions required to access a QPG . . . . . . . . 48

4.1 Workload-driven partitioning methods supporting online graph queries 65

5.1 Case studies for the aggregate-oriented NoSQL stores . . . . . . . 82

5.2 (a) specification of QPG nodes, (b) QPG edges and (c) QPG queries in the digital library . . . . . . . . . . . 86

5.3 (a) specification of QPG nodes, (b) QPG edges and (c) QPG queries in the online store . . . . . . . . . . . . . . . . 90

5.4 (a) specification of QPG nodes, (b) QPG edges and (c) QPG queries in the the easy anti cheat . . . . . . . . . . . . 95

5.5 Dataset statistics in millions $(\mathrm{M}) \ldots \ldots . \ldots . \ldots 97$

5.6 Workload runtime $(\mathrm{sec}) \ldots \ldots \ldots$. . . . . . . . . . 102 


\section{List of Figures}

1.1 The major database technologies and some corresponding systems . . 5

1.2 A balanced min-cut example. . . . . . . . . . . . . 9

1.3 The arrival rate of top four queries in BM query log. . . . . . . . . 10

1.4 The adopted design science research methodology process model . . . 12

2.1 Four major categories of NoSQL stores regarding their data models . 19

3.1 Conceptual schema of a sample e-commerce platform . . . . . . . . 34

3.2 (a) a KV logical schema that satisfies both the queries $q_{2}$ and $q_{3}$ in our running example; and (b) some instances of the schema . . . . . . . 36

3.3 (a) A CF logical schema that satisfies both the queries $q_{2}$ and $q_{3}$ in our running example; and (b) the corresponding instance . . . . . . 38

3.4 Document collection schemas that satisfy $q_{1}$ in our running example, by either (a) embedded documents or (b) joined documents . . . . . 40

3.5 An overview of our method for designing NoSQL database schemas . 42

3.6 (a) the query path of $q_{3} ;(\mathbf{b})$ a SQL-based syntax of $q_{3} \ldots \ldots 44$

3.7 (a) the paths of queries in the workload of the running example; (b) the corresponding QPG. . . . . . . . . . . 46

3.8 The resulting KV schemas of mapping QPG in the running example . 48

3.9 The resulting CF schemas of mapping QPG in the running example . 51

3.10 The graph of query paths whose entry point is Order . . . . . . . 57 
3.11 (a) the resulting document collection schemas of mapping QPG in the running example; (b) the same mapping with respect to Order as an

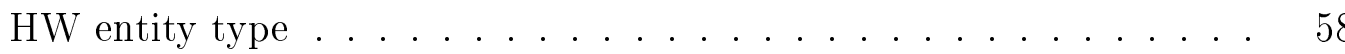

4.1 (a) existing partitions before reassigning vertex $v_{3}$; (b) the improved quality of partitions after reassigning $v_{3}$ from the $2^{\text {nd }}$ to the $3^{\text {rd }}$ node

4.2 (a) a high-degree active vertex $u$ regarding $Q$ as a query pattern belonging to the existing workload; (b) splitting up the edges of $u$ across the nodes $\ldots \ldots \ldots \ldots \ldots \ldots \ldots \ldots \ldots \ldots$

4.3 (a) system architecture; (b) graph-based SPARQL query processing

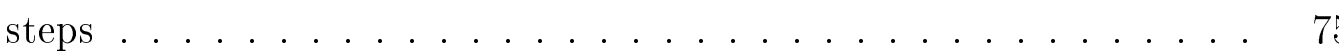

5.1 (a) the digital library's entity graph; and (b) the corresponding workload 84

5.2 (a) the paths of queries in the workload of the digital library; (b) the corresponding $\mathrm{QPG} \ldots \ldots \ldots \ldots \ldots \ldots \ldots$

5.3 The resulting CF schemas of mapping QPG in the digital library . . 87

5.4 Response times in our design vs. Chebotko et al. . . . . . . . . 88

5.5 (a) the online stores' entity graph; and (b) the corresponding workload 88

5.6 (a) the paths of queries in the workload of the online store; (b) the

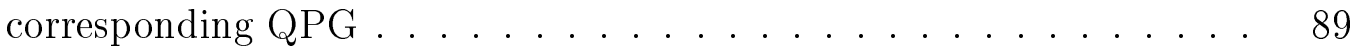

5.7 The resulting document collection schemas of mapping QPG in the online store, with control on the denormalization degree of the Product entity type . . . . . . . . . . . . . . . . . 91

5.8 Response times in our design vs. de Lima's . . . . . . . . . . . . . 92

5.9 (a) the the easy anti cheat's entity graph; and (b) the corresponding workload . . . . . . . . . . . . . . . . . . . . 93

5.10 (a) the paths of queries in the workload of the easy anti cheat; (b) the

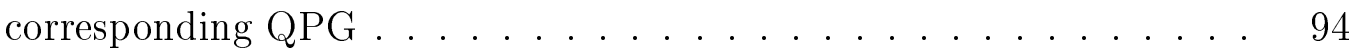

5.11 The resulting CF schemas of mapping QPG in the the easy anti cheat 95 
5.12 Response times in our design vs. Mior et al. . . . . . . . . . . . . 96

5.13 IPT ratio regarding the six workloads . . . . . . . . . . . 100

5.14 Load imbalance factor regarding the three static workloads . . . . . . 101

5.15 Sensitivity analysis of reassignment threshold and edge log size . . . . 103

5.16 Average number of replicas under different splitting thresholds . . . 105

5.17 WASP adaptivity to dynamic workloads . . . . . . . . . . 106

5.18 WASP scalability regarding the WatDiv dataset . . . . . . . . 107 


\section{Nomenclature}

ACID Atomicity, Consistency, Isolation and Durability

BIP Binary Integer Programming

BM British Museum

CF Column Family

CMS Content Management System

DHT Distributed Hash Table

DSRM Design Science Research Methodology

EER Extended Entity-Relationship

ER Entity-Relationship

ES ElasticSearch

HU Highly Used

HW Highly Written

IPT Inter-Partition Traversal

KV Key-Value

LSM-tree Log-Structured Merge-tree 
MHE McGraw-Hill Education

N1QL Non-first normal form Query Language

NoSE NoSQL Schema Evaluator

NoAM NoSQL Abstract Model

QPG Query Path Graph

RDBMS Traditional Relational Database Management System

RDF Resource Description Framework

SSN Social Security Number

SCF Super Column-Family 


\section{Chapter 1}

\section{Introduction}

\subsection{Why NoSQL?}

Since the early 2000s, the advances in the Web technology, social network, mobile devices, and the internet of things have resulted in the explosion of structured, semistructured, and unstructured data that are managed by big data applications [1]. Such applications have a variety of requirements from database systems, mainly, (1) horizontal scalability, that is viable through data partitioning, to linearly adapt to the massive amounts of data and the increasing rate of query processing by making use of additional resources, (2) high availability and fault tolerance to respond to client requests even in the case of hardware/software failure or upgrade events, (3) ACID properties [2] to support strongly consistent data, and (4) database schema maintainability to reduce the cost of schema evolution.

Achieving the above requirements through RDBMS is very difficult or even unattainable. First, the predefined relational schema makes the evolution of relational databases costly due to the complex data transformation/migration so this technology cannot satisfy agile and highly iterative application development approaches required by the existing processing scenarios of big data. Second, scaling up these systems necessitates their movement to stand-alone servers with more enhanced hardware; that 
is a costly process and incurs significant system unavailability during each movement. Third, by scaling out (i.e., employing more commodity servers) these systems even in a single data center, the complexity and overhead of joining distributed normalized data are increased; in addition, these systems encounter availability and performance issues due to the use of distibuted ACID transactions [3,4]. As an illustration, assume a distributed storage system that stores a data item $D$ replicated on three nodes $N_{1}$, $N_{2}$ and $N_{3}$, and a communication fault that splits the network (of the nodes) into two sub-networks: $\left\{N_{1}, N_{2}\right\}$ and $\left\{N_{3}\right\}$. If a modification request on $D$ is submitted to $N_{3}$, then there are two possible scenarios. First, the request is successfully completed, knowing that by healing the partition the modified value of $D$ is propagated to its replicas in $N_{1}$ and $N_{2}$. This scenario chooses the availability of the request. However, it may result in inconsistent values of $D$. Second, the request is aborted, knowing that contacting with $N_{1}$ and $N_{2}$ is not possible until healing the partition. This scenario chooses the strong consistency of data item $D$. However, it results in the unavailability of the request. This simple example raises a question whether both the strong consistency of data and availability of requests can be achieved simultaneously.

This trade-off was firstly observed by Rothnie and Goodman [5]. However, increasing the commercial popularity of the Web along with the growing demand for the geographic replication of data and high availability of operations motivated Fox and Brewer [6,7], to reclaim this trade-off as CAP principle. This principle indicates that at most two of the three desirable properties Consistency, Availability and Partition tolerance can be achieved simultaneously by a distributed data store. Later on Gilbert and Lynch [8,9] formalized and proved $C A P$, that became known as the $C A P$ theorem. In this context, the $C A P$ properties are defined as below:

- Consistency is viewed as a qualitative property denoting linearizability. Based 
on this definition, the $C A P$ consistency is not a dynamically observed metric determined by the operational status of the system. Instead, it is statically determined with regard to the system's employed algorithms whether they guarantee linearizability or not.

- Availability is viewed as a qualitative property denoting every request sent by a client eventually (within a finite time) receives a successful (non-error) response. Based on this definition, the "availability" or "unavailability" of a system is statically determined with regard to its used algorithms. However, this definition has some ambiguities. First, some systems that are highly available due to their high uptime, may not be considered as CAP-available. For example, a distributed data store that uses a quorum-based synchronous replication is not $C A P$-available because during a network partition, read/write operations on the minority side of the partition may not be able to successfully complete. Second, there is no bound on the response time. For instance, an operation that is successfully completed after one week, is considered CAP-available. However, such an operation is unavailable according to the instinctive notion of availability. In other words, "latency" (response time), that is practically an important feature, is not taken into account in this definition.

- Partition tolerance is viewed as a qualitative property (of system's employed algorithms) denoting that even in the presence of a network partition the system continues providing its CAP-availability or CAP-consistency guarantee. This definition is also fuzzy and unclear, as network partitions are not the only failures in a distributed system. In other words, there are also other failures such as lost messages and node failures.

Accordingly, by forfeiting any one of the discussed $C A P$ properties, distributed data stores are categorized as follows: 
- Consistency + Availability - CA systems. The algorithms used by $C A$ systems do not have any assumption about network partitions. Consequently, achieving this combination is practically impossible in distributed systems, as the occurrence of network partitions is inevitable. Therefore, the fundamental CAP trade-off is between consistency and availability. This trade-off became a justification for supporting weak consistencies and justifying the design decisions of distributed data stores, where consistency is sacrificed more than availability.

- Consistency + Partition tolerance $-C P$ systems. This combination is achieved by distributed data stores that preserve CAP-consistency. However, in case of a network partition, a read/write request may not be responded due to avoiding the risk of inconsistency. Therefore, $C P$ makes sense for systems designed to operate in a reliable network such as a single datacenter due to the infrequency of network partitions.

- Availability + Partition tolerance - AP systems. This combination is achieved by distributed data stores that enforce a weak notion of consistency. However, the execution of conflicting writes is allowed, which may result in the divergence of replicas and necessitates implementing a conflict resolution mechanism. These systems are typically used by applications whose users are geographically scattered in a wide-area network and require a high level of availability along with a fast response time (instead of strong consistency) such as web caching.

The above categorization has resulted in an emerging trend of non-relational data stores, called NoSQL stores $[10,11]$, that aim at satisfying the high availability and scalability requirements of big data applications (see Figure 1.1). NoSQL is an acronym for "Not Only SQL" since some of these systems support SQL-like queries. According to [11] contemporary database systems are referred to as "data stores" where more flexible data models are used and DBMS functionalities may not be fully 


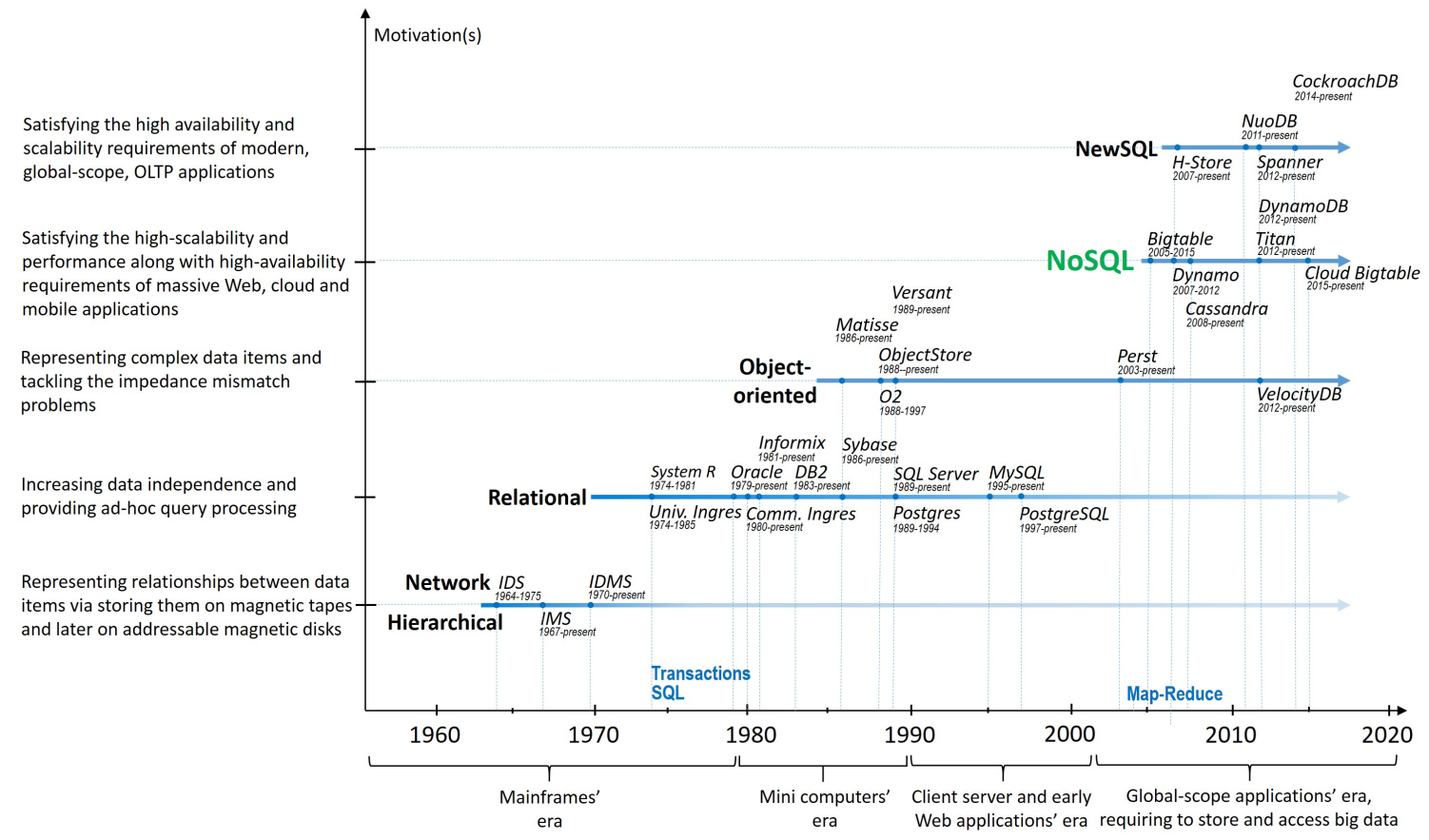

Fig. 1.1. The major database technologies and some corresponding systems

provided. NoSQL stores have some design characteristics in common:

- On the contrary to the traditional database technologies that support schemafull data models, NoSQL stores adopt more flexible data models that can be schema-less $^{1}$, and data may need to be interpreted at the application level.

- Weak consistency transaction models are allowed by relaxing the strict ACID properties. This allows NoSQL stores to scale out while achieving the high availability and low latency required by Web-based applications.

- By placing relevant data items together in the same storage node and using lots of data duplication, NoSQL stores facilitate joining data as there is no need to move related data over the network, which allows them to achieve better query performance. However, this may lead to costly updates on duplicated data.

1 A schema-less data model allows data to have arbitrary structures as they are not explicitly defined by a data definition language (schema-on-write). Instead, they are implicitly encoded by the application logic (schema-on-read). 
- There is a clever use of distributed indices, hashing and caching for data access and storage.

- Most of them are cluster and datacenter friendly. In other words, data can easily be replicated and horizontally partitioned across local and remote servers.

- They provide Web-friendly access through a simple client interface or protocol to query data.

These features make NoSQL stores not as a revolutionary replacement for the current relational database systems, but as a remedy of their shortcomings for handling big data. So far, more than 200 different NoSQL stores have been reported and the list is still increasing ${ }^{2}$.

\subsection{NoSQL database schema design problems}

NoSQL systems utilize different data models for different purposes, including (among others) KV, wide-column and document models. Despite their differences, the three data models are aggregate-oriented. In more detail, they organize data as units of related KV pairs in a nested way. Such a nested data unit represents data that are accessed (read/write) together in one operation $[12,13]$. Hence, they can facilitate costly join operations through embedding data [14,15]. This means denormalizing 1:N or $M: N$ relationships by clustering related entities.

To tap into the benefits of data nesting or denormalization in designing the schemas of aggregate-oriented NoSQL databases, designers should not only consider which data are going to be stored in the database but also how such data are going to be accessed [15]. However, NoSQL stores were initially lacking in well-defined methods for database schema design. On the other hand, despite existing wellestablished methods of traditional database schema design, where even some are

2 https://nosql-database.org/ 
workload-driven [16-19], they do not fit NoSQL stores. This is mostly because they aim to achieve other targets such as ACID properties. To address this gap, many methods have been proposed for the design of aggregate-oriented NoSQL database schemas over the last several years. Nevertheless, these methods still suffer from some drawbacks which can be summed up as follows.

- A majority of methods focus on a specific NoSQL data model, where each one provides its own conceptual modeling approach. This implies an extra effort for designers who work with different NoSQL data models and need to change between design methods. For example, a designer who is either developing a multi-model NoSQL database [20], or migrating an old NoSQL schema to a new one [21] has to specify the same conceptual schema and workload in different notations. Although some approaches provide the basis for designing different aggregate-oriented NoSQL database schemas, they do not take into account the specific features of different NoSQL data models, which may impact the quality of generated database schemas.

- Many methods detail how a NoSQL database schema can be constructed using a series of heuristics and guidelines that must be manually applied. This might be time consuming and error-prone. Although some methods address the automation of the design process, designers cannot customize them so that any design trade-off can be treated differently regarding the requirements of each context. Note that, though data denormalization might increase the performance of read operations, there are downsides to it as well. In more detail, as denormalizing data increases data redundancy, write operations might be impacted due to the consistency maintenance overhead. Storage overhead might also be increased. Therefore, it would be beneficial to develop a design process that balances these contradictory context requirements effectively. 


\subsection{NoSQL graph partitioning problems}

Despite the aforementioned aggregate-oriented NoSQL data models that focus on storing information about entities, the graph NoSQL data model focuses on the efficient traversal of entity relationships (or edges) using an appropriate partitioning of graph entities (vertices) across servers. So far, a significant number of graph partitioning methods have been proposed to address online graph queries ${ }^{3}$ [22-32]. However, there are still several significant shortcomings.

- The existing strategies are mostly workload-agnostic, as they presume the same probability of traversing edges or visiting vertices of the graph, which does not always hold with different workloads. In other words, they do not consider frequent query patterns and locality of access to graph elements, which may degrade system performance. For example, Figure 1.2 depicts a simple property graph and workload. After running a vertex-centric partitioner, we get an optimal partitioning $\{\mathrm{P} 1, \mathrm{P} 2\}$. However, it is not optimal for the workload. Each query may require an expensive inter-partition traversal because of the cutting edge "?x authors ?y".

- The existing strategies are mostly graph topology-agnostic, as they do not differentiate between high-degree and low-degree vertices. In a hybrid-cut model, the vertex-centric partitioning is exploited for low-degree vertices while incident edges of high-degree vertices are partitioned via the edge-centric partitioning. Although some graph analytics engines exploit the hybrid-cut model, no workload-driven partitioning strategy has yet exploited this model.

- Many existing workload-aware strategies are unable to adapt to dynamic workloads. Although some strategies exploit the query log to extract frequent query

$\overline{3}$ As offline graph analytics iteratively access the entire graph, we only focus on online graph queries which usually access a fraction of the graph. 


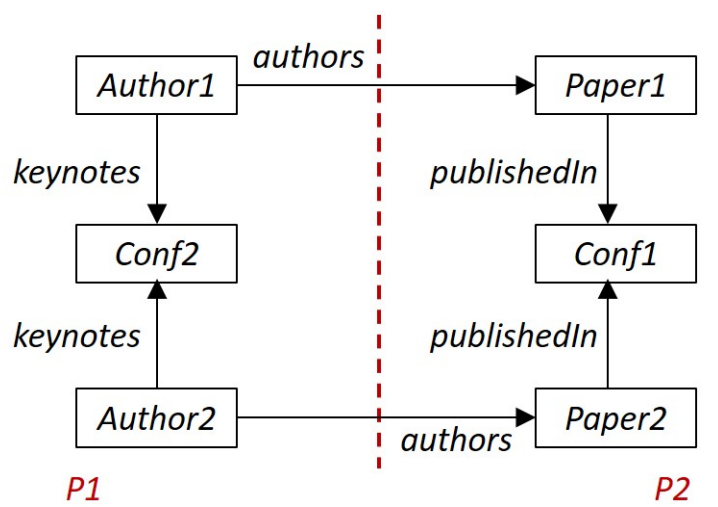

$W=\{\langle Q 1,90 \%\rangle,\langle Q 2,10 \%\rangle\}$

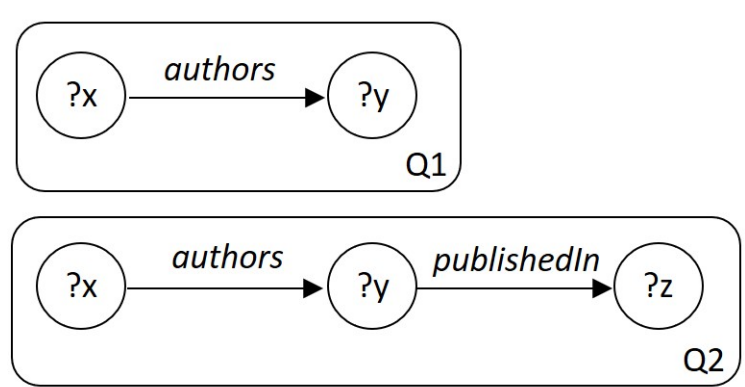

Figure 1.2. A balanced min-cut example.

patterns, whereby the associated triple patterns are partitioned to increase access locality, such strategies suffer from two drawbacks: (1) Over time, the popularity of frequent query patterns may fluctuate. Accordingly, we have done some research on the BM query log [33]. It spans from April 12, 2014 to October 16, 2014 and records over 1.2 million query requests. The daily arrival rate of the top four frequent queries is demonstrated by Figure 1.3. We have added the frequencies of the four queries together as they follow a similar arrival trend. As we can see, despite being frequent (over 150 times a day) during a short period from August 4, 2014 to August 22, 2014 these queries are infrequent most of the time. Therefore, a partitioning plan based on frequent query patterns with temporary spike results in inefficient queries most of the time. (2) Over time, the existing frequent query patterns may be outdated. In other words, queries in the future can rarely be reflected by the query logs in the past.

\subsection{Thesis aims and objectives}

This thesis mainly aims at designing a framework that addresses the aforementioned challenges of designing the schema of aggregate-oriented NoSQL datasets and the 


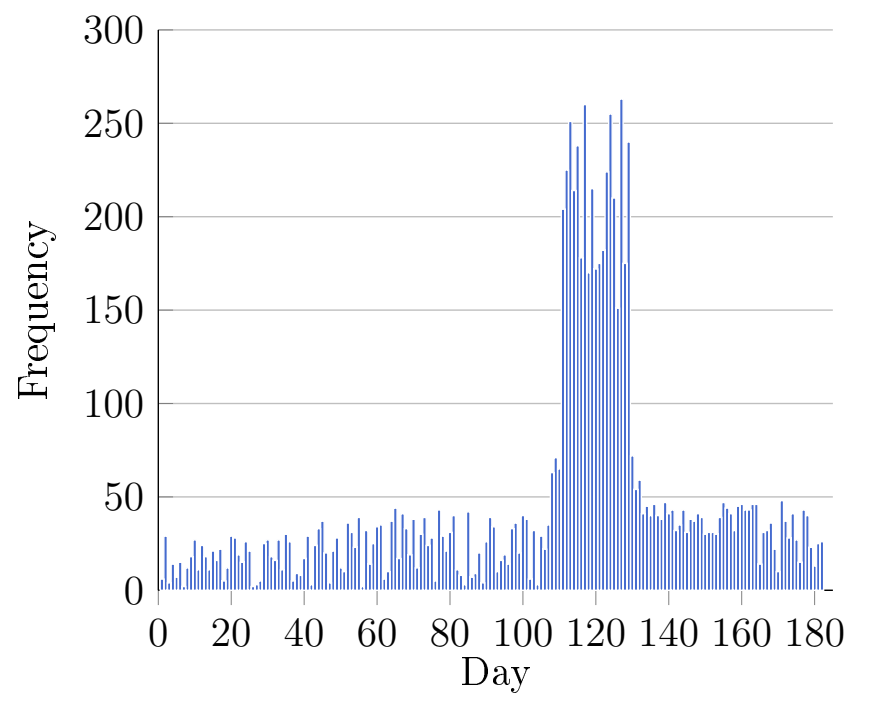

Figure 1.3. The arrival rate of top four queries in BM query log.

partitioning of graph NoSQL datasets. It is worth mentioning that NoSQL data modeling and partitioning are two non-orthogonal concepts. In more detail, any strategy that is used for modeling the aggregate-oriented datasets affects their partitioning. Accordingly, we present a generally applicable solution for the design of aggregate-oriented NoSQL database schemas from the same conceptual model, that can also be tailored to specific needs regarding the workload information. Note that generalizing an existing method is challenging as the strategy used by each method, to map from the input conceptual schema to a database schema, tightly depends on the characteristics of a specific NoSQL data model. However, our approach exploits a generic NoSQL data model that is based on the "nested data unit" concept as a common characteristic of aggregate-oriented NoSQL data models.

On the other hand, for the partitioning of graph NoSQL datasets, we present a workload and topology adaptive partitioning method named Helios. Specifically, Helios incrementally adjusts partitions (initially obtained by the random hashing of graph vertices) to the existing workload. In more detail, during the existing workload, a subset of vertices called active vertices and a subset of edges called active edges are 
frequently visited and traversed, respectively. In this sense, our partitioner distributes active vertices across partitions proportional to their frequency of visits. This leads to balance in the existing computational load, which in turn increases the throughput. On the other hand, it reduces the probability of the cut of active edges proportional to their frequency of traversals. As such, active vertices belonging to the same query are likely collocated into the same partition, which in turn decreases the query response time. In addition, Helios utilizes a hybrid-cut model whereby the exploration of high-degree vertices is distributed across multiple nodes, which in turn increases the throughput.

\subsection{Research methodology}

Design science is an outcome-based research methodology that offers specific guidelines for designing and evaluating purposeful innovative artifacts to solve a special problem in a particular domain [34]. An outcome artifact is perceived to be the knowledge that ranges from novel methods, algorithms, and tools, to assumptions about the context wherein the artifact is intended to function. To form a novel contribution, the research outcome must either solve a problem that is not solved yet or provide a better solution for it. In this thesis, DSRM is regarded as an optimal methodology to follow for fulfilling the research aims described in Section 1.4. Accordingly, we adopt the DSRM introduced by Peffers et. al. [34]. Figure 1.4 depicts the steps that compose our adopted DSRM, and each step is explained and related to the thesis chapters as follows.

1. Problem identification and motivation. This step involves critical thinking of the research problem and modeling strategies to justify the value of designing a solution to solve that problem. In the first phase of this step, our aim is to identify the gaps in the related literature as presented in Sections 1.2 and 1.3. 


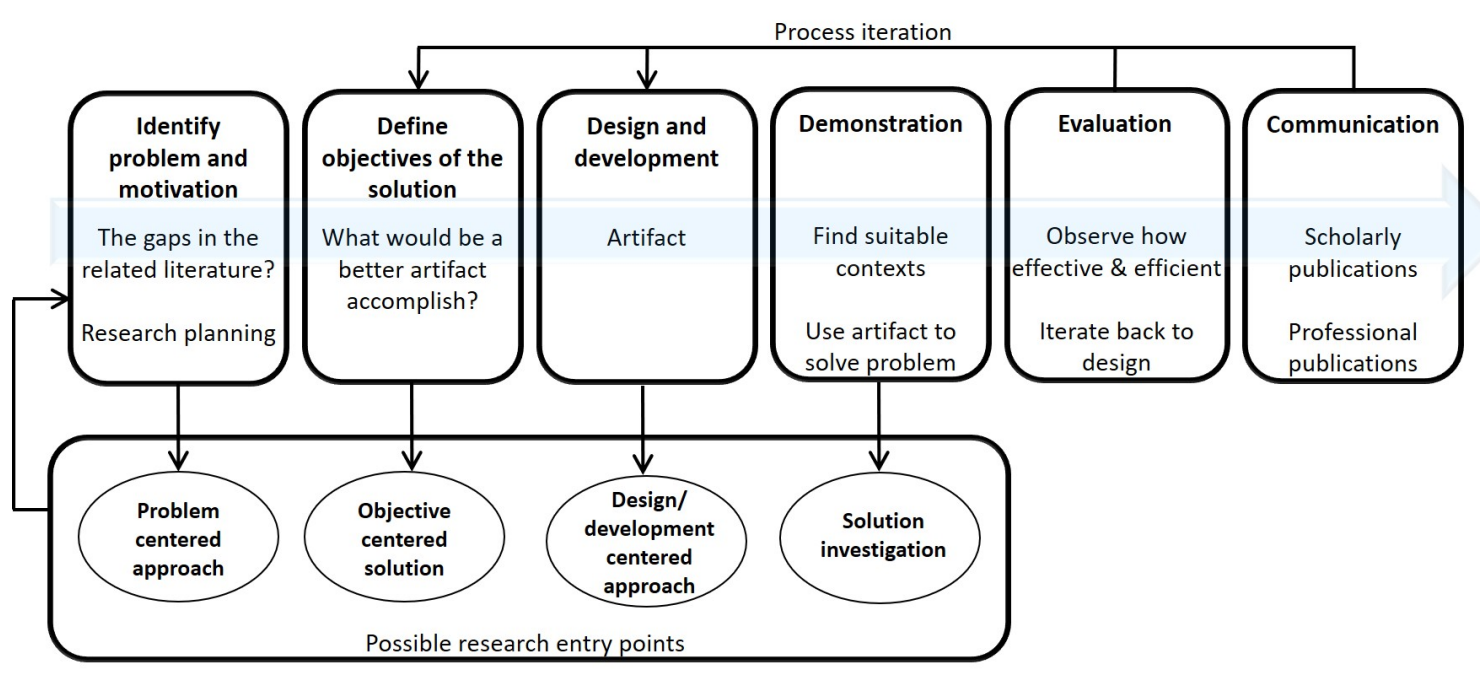

Fig. 1.4. The adopted design science research methodology process model

In the second phase, the research aims are carefully articulated as presented in Section 1.4. The third phase requires the selection of NoSQL data models and the tools for developing the solution. The last step involves research planning by dividing the research problem into two sub-problems, namely:

- Workload-driven modeling of aggregate-oriented NoSQL database schemas.

- Workload-driven partitioning of graph NoSQL datasets.

In the last step, we also identify the required means to solve each sub-problem.

2. Objectives of the Solution. This step requires knowledge of the state of the problem, its current solutions, and their efficacy. The problem definition in the previous step is used to propose the objectives of the solution. Our research problem is general in distributed database systems, where one of the challenges is data partitioning. However, this problem in the context of our research exhibits a fundamental difference in that its solution has specific objectives as the following. 
- The solution is NoSQL-oriented, whereby it utilizes data denormalization to facilitate joining data.

- The solution is workload/topology-aware, whereby it exploits the existing workload to increase query performance

- The solution is generally-applicable for different NoSQL data models, as it considers the same strategy for modeling aggregated-oriented NoSQL datasets or partitioning property graphs.

3. Design and development. This step aims to design and develop a solution for the defined problem. The details of this step are covered in chapters 3 and 4. The design of the component responsible for workload-driven modeling of aggregate-oriented NoSQL database schemas is presented in Chapter 3, and the design of the component responsible for workload-driven partitioning of graph NoSQL datasets is presented In Chapter 4.

4. Demonstration. This step involves using the developed framework in suitable contexts. In this thesis, three case studies including a digital library, an application in e-commerce, and a cheat detection system for multiplayer games are used for evaluating our aggregate-oriented database schema modeler. Moreover, WatDiv and DBpedia RDF datasets are used for evaluating our graph partitioner.

5. Evaluation. This step observes and measures how well the proposed framework resembles an optimized solution to the defined problem. It involves assessing the effectiveness/efficiency of the components in the proposed framework compared to their current state-of-the-art counterparts available in the relevant literature. In DSRM, once framework components are developed, researchers start a thorough testing process for each component. In this thesis, we righteously evaluate the main components of the proposed framework in Chapter 5. The evaluations 
aim to assess the efficiency of each component, and its effectiveness compared to the chosen state-of-the-art approaches.

6. Communication. The main thesis contributions are established based on three journal papers published in ACM CSUR and Springer DSE journals. A short conference paper has also been published in ACM SIGMOD.

\subsection{Thesis contributions}

Our contributions regarding the generation of aggregate-oriented NoSQL databases are as follows.

- We propose a novel generic NoSQL logical model that takes into account the conceptual model and workload of the system. This model is based on the "nested data unit" concept as a common characteristic of aggregate-oriented NoSQL data models.

- We present a set of rules to map the generic model to specific logical schemas of targeted NoSQL data models regarding their important features and design trade-offs between the read query performance and storage overhead or consistency maintenance.

- We implement a workload-driven NoSQL database schema generator that automates the generation of various aggregate-oriented database schemas according to a generic model. It generates some scripts which instantiate the resulted database schemas, with respect to targeted NoSQL technologies.

Our contributions regarding the partitioning of graph NoSQL databases are as follows.

- We propose a dedicated cost model to manage vertex reassignment according to frequent query patterns and graph topology. The model decides which vertex should 
be moved and where to move in order to maximize the reassignment gain. Simultaneously, the load-balance on each computing node is preserved.

- We introduce an incremental lightweight metadata management, which exploits Redis [35] as a fast in-memory database. Hence, the overhead of metadata maintenance only accounts for a small portion of the total runtime. Redis incrementally stores the states of active vertices in each node in the form of key-value pairs.

\subsection{Thesis outline}

In this thesis, we investigate and evaluate automated solutions for modeling and partitioning NoSQL datasets. We present techniques that are related to the two nonorthogonal hot topics, namely designing aggregate-oriented NoSQL database schemas and NoSQL graph partitioning. In particular, we examine new and exciting methods and algorithms in order to introduce a workload-driven framework for modeling and partitioning NoSQL datasets, which can be generalized in the future for reusability in other emerging database technologies such as NewSQL stores [36]. The outline of this thesis is as follows.

Chapter 2 illustrates NoSQL data models and discusses the related literature from the perspective of our research objectives. This chapter starts by giving the latest categorization of NoSQL data models and explores their characteristics [10]. We then review various data partitioning approaches and frameworks that have been proposed for different NoSQL data models. Finally, the general limitations of existing works as well as their fundamental drawbacks are discussed.

Chapter 3 presents our generally-applicable solution for designing aggregate-oriented NoSQL database schemas. In this chapter, we first describe our proposed generic NoSQL data model. Next, we discuss how this model is mapped to specific logical 
schemas, regarding some design trade-offs.

Chapter 4 presents our solution for the steaming partitioning of property graph datasets. This chapter starts by explaining vertex activity weights and edge traversal weights that encode workload characteristics. The chapter then discusses our workload-adaptive and topology-aware vertex reassignment strategy.

Chapter 5 presents the empirical evaluations of the methods and algorithms proposed in this thesis. As per the adopted research methodology, we focus on evaluating the effectiveness of our methods using various datasets. To evaluate the efficiency, we compare the overall performance of our methods against state-of-the-art NoSQL database schema generation and data partitioning methods.

Chapter 6 presents the conclusions drawn from this research, and discusses the possible research directions which our future work might take. 


\section{Chapter 2}

\section{Literature Review}

As part of the followed DSRM methodology [34], this chapter discusses the characteristics of NoSQL data models and introduces the literature of data partitioning for such models. The presented discussions help in understanding the cost implications of adopting existing approaches of NoSQL data partitioning. They also pave the way for introducing our proposed solutions for the two tightly coupled NoSQL database design and data partitioning and delineating how they advance on the current stateof-the-art research. Though this chapter mainly focuses on exploring various NoSQL data partitioning approaches, additional reviews related to the downstream methods of designing aggregate-oriented NoSQL database schemas and partitioning graph databases are covered in Chapters 3 and 4.

\subsection{NoSQL data modeling}

A data model specifies how real-world entities and their relationships are represented and operated [37]. Accordingly, NoSQL stores are mainly categorized into key-value, wide-column, document and graph stores. Each of these models is characterized by a number of features that make the corresponding stores suited to specific application scenarios. 


\subsubsection{Key-value stores}

KV stores are the simplest and most popular NoSQL stores, where data are managed and represented as KV pairs and the value part is uniquely identified by an indexed key part. The key part is either simple (e.g., a URI, hash or filename) or structured such as composite keys. It may also be system generated or application-defined. The value part represents data with an arbitrary type, structure and size, that is encoded as a byte array, such as BLOB, where its serialization/deserialization is left to the client application. Due to the schema-less structure of stored values, their indexing and querying must be implemented by the client application. Therefore, KV stores are just suitable for applications that only use a single key to access data such as online shopping cart, user profile/configuration, and web session information. This simple data model results in the easy partitioning and efficient querying of data, which in turn leads to the high scalability of KV stores.

In practice, since many applications require a value-based lookup of data, advanced KV stores provide additional functionalities such as indexing and querying the content of values of specific data types. For example, Redis $^{1}$ and Aerospike ${ }^{2}$ support the list data type. They allow performing atomic operations on list values such as pushing into a list without replacing the entire value. In addition, Riak $\mathrm{KV}^{3}$ supports document types such as JSON. These extra functionalities have blurred the border between KV stores and other kinds of NoSQL stores. For example, Riak KV can be considered as a document store.

A typical KV store provides a simple set of key-based query operations such as get(key), put(key, value) and delete(key). Get(key) retrieves a value (or a list of values with different versions) associated with the key. Put(key, value) adds the KV

\footnotetext{
https://redis.io

https://aerospike.com/

https://basho.com/products/riak-kv/
} 
(a). Using the basic KV data model for a health management system

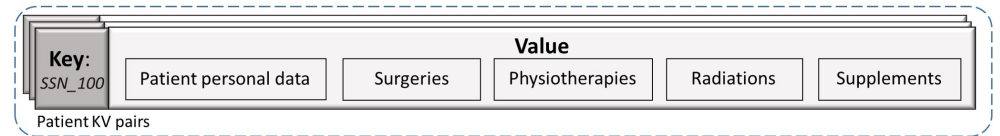

(b). Using the wide-column data model for the Facebook Inbox Search

(c). Using the document data model for the McGraw-Hill Education

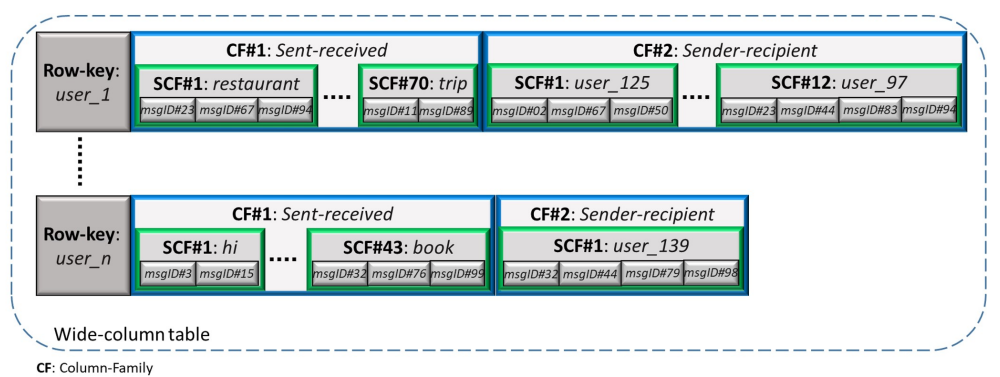

CF: Column-Family
SCF: Super Column-Family

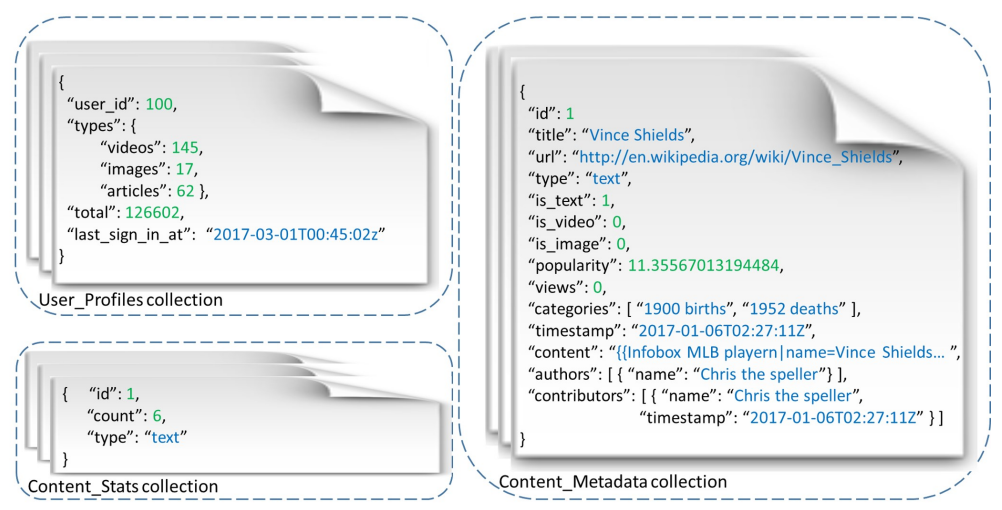

(d). Using the graph data model for the social network of Facebook. As an illustration, suppose David along with his friend Sara visit the Eiffel Tower. David uses his cellphone to record this visit by 'checking in' to the Eiffel Tower and tagging Sara to let other friends know that she is also there. Jack writes a comment on this and John likes it.

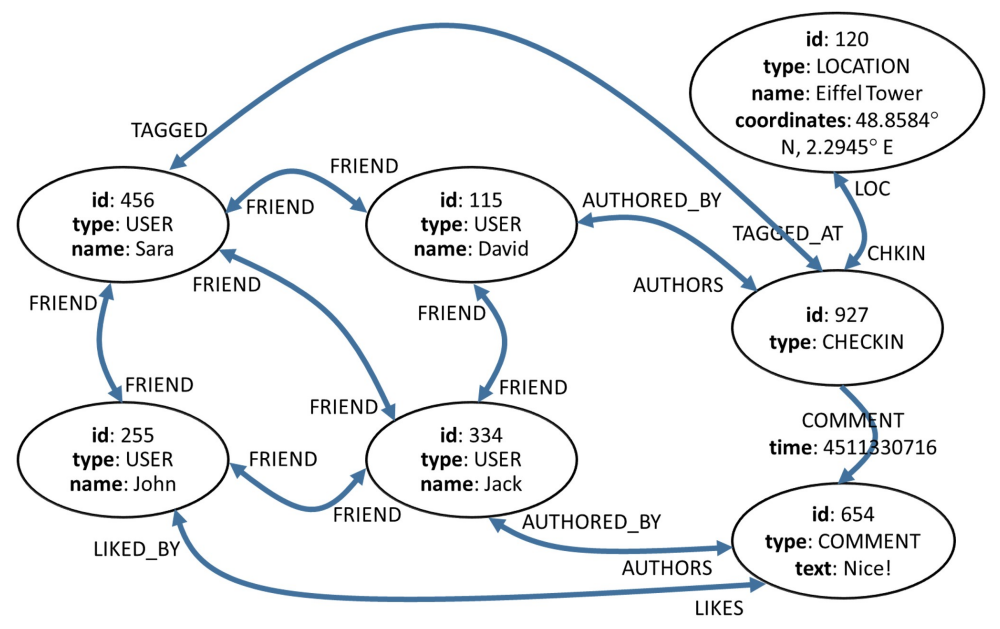

Fig. 2.1. Four major categories of NoSQL stores regarding their data models

pair to the store only if the key is not present in it. Otherwise, the stored value is updated with a new version. Note that updating even a part of a stored value requires replacing the whole value. Delete(key) removes the key and its associated value(s). 
The details of the above operations depend on some factors such as consistency model, indexing, etc. These single-key operations do not allow manipulating multiple values with one operation. These operations can be easily performed through the REST [38] or Lucene ${ }^{4}$ interfaces. Figure 2.1(a) shows a sample basic KV store used by a health information management system, assuming that the medical information of patients are mainly queried based on their SSNs and patient's data are rarely modified.

\subsubsection{Wide-column stores}

These are extended KV stores where the value part is represented as a table of nested KV pairs with a flexible schema. In more detail, a table is a collection of rows, where each row represents a highly structured construct that is uniquely identified by a row key and consists of a fixed number of CFs. A CF contains an arbitrary number of columns or cells that are logically related to each other and usually accessed together regarding query requirements. This justifies why data in wide-column tables are physically stored per CFs instead of rows. The flexible schema of a CF is viable through adding or removing its columns at runtime, where a column has a name and a value with a simple (e.g. integer or string) or a complex structure such as a collection of columns.

Wide-column stores usually allow storing a configurable number of versions of each cell value, indexed by timestamps; and a value is retrieved through a triple $<$ rowkey, column-key, timestamp $>$. A timestamp is either automatically assigned by the store, or explicitly specified by the client application. Wide-column stores offer more extended client interfaces than KV stores, as their indexing and querying facilities are based on various constructs such as rows, CFs and columns.

Figure 2.1(b) shows an example of how Facebook used a wide-column table for its Inbox Search service. This service enables the user to search through her

$4 \quad$ https://lucene.apache.org 
sent/received messages based on either a keyword (called term query) or the name of a sender/receiver (called interaction query). More precisely, for both term and interaction queries the user-ID is the row-key. Sent-received and Sender-recipient are two different CFs that satisfy the requirements of term and interaction searches respectively. In Sent-received, the keywords that make up the user's messages become nested CFs that are also called SCFs. In each SCF, the individual message-IDs (or links to messages) become the columns, which in turn minimizes redundancy. Similarly, in Sender-recipient, the user-IDs belonging to all senders/recipients of the user's messages become SCFs.

Data in wide-column stores can be efficiently partitioned horizontally by rows and vertically by CFs, that makes them suitable for storing huge datasets. Note that many wide-column stores, such as Apache Cassandra [39], Apache HBase ${ }^{5}$ and Google BigTable [40], use LSM-trees [41] to implement a highly efficient storage backbone per CF.

The high scalability and flexibility along with the support of MapReduce tasks [42] (for the parallel processing of large datasets) make wide-column stores suitable for analytical applications. However, the predefined set of CFs, makes it difficult to use wide-column stores for applications with evolving schemas [43,44].

\subsubsection{Document stores}

These are extended KV stores where the value part is represented as a document of nested KV pairs with a flexible schema, that is encoded in standard semi-structured formats such as XML or JSON. In more detail, a document is a set of properties where each property has a name along with one or more values. The value part can be either primitive or complex such as an embedded document or a collection of properties. The flexible schema of a document is viable through adding or removing

$5 \quad$ https://hbase.apache.org/ 
its properties at runtime. Unlike the opaque content of values in KV stores, document stores know the format of documents and support indices and search functionalities based on their property names and values.

Document stores fit applications whose data can be simply represented in a document format such as CMS and blogging platforms. For example, a blog post including various (nested) properties, such as tags, comments, images and videos, can be easily represented in a document format. These stores are also suitable for the high development productivity and low maintenance cost of modern Web 2.0 applications, for two main reasons. First, these applications have a constant evolution of data schema and benefit from the flexible data model of document stores. For example, consider a monitoring application that stores and analyzes log data from various sources, where each source generates different data. Evolution to new log formats can be easily enabled by the flexible schema of documents. However, such an extension would be costly in relational databases, as it needs the addition of new columns to the existing tables or the creation of new tables for new formats. Second, Web 2.0 applications support data models such as JSON with tight integration with popular programming languages such as Python, JavaScript and Ruby. This has radically decreased the impedance mismatch $[45,46]$ between these programming languages and document stores, as object-oriented constructs can be easily mapped to documents.

Some document stores, such as MongoDB $^{6}$ and Couchbase Server ${ }^{7}$, provide an additional construct called collection or bucket that contains a set of documents representing the same category of information. Such collections look like tables in relational databases, where each row is a document with a unique key but not necessarily with the same schema as others. By using collections, features such as resources, replication, persistence and security can be managed for each group of documents instead

6 https://mongodb.org/

7 https://couchbase.com/nosql-databases/couchbase-server 
of individual ones. Figure 2.1(c) shows an example of how MHE used the document data model for building a self-adapting and interactive learning portal that delivers personalized search results ${ }^{8}$. It integrates Couchbase Server ${ }^{7}$ and $\mathrm{ES}^{9}$ to handle the full-text search and content discovery. The vision of MHE divides a textbook into media objects including articles, images and videos. Data are stored in JSON documents and categorized into several collections including 1) Content_Metadata that stores media objects' metadata along with the content of text articles, 2) User_Profiles that stores user view details per media object, used for customizing ES search results based on user preferences, and 3) Content_Stats that stores the view details of each media object, used for boosting ES search results based on document popularity.

Document stores allow querying data inside a document without having to retrieve the whole document and inspecting it at the application level. For example, according to Figure 2.1(c), the following query shows how to use the SQL-like N1QL ${ }^{10}$ in Couchbase to search for the documents of the collection Content_Metadata. This query filters the documents by the property title that has the value "Vince Shields", and then returns url and categories property values.

SELECT url, categories FROM Content_MetaData

WHERE title $=$ 'Vince Shields'

Note that native XML stores (e.g., Marklogic Server ${ }^{11}$ and Virtuoso ${ }^{12}$ ) are the predecessors of modern JSON document stores. They implement a variety of XML tools and standards (e.g., XQuery ${ }^{13}$ ) for XML view, storage, keyword search [47],

8 https://youtube.com/watch? $=0 \mathrm{~m} Q t 5 \mathrm{gEOIhI}$

9 https://github.com/elastic/elasticsearch

10 https://www.couchbase.com/products/n1ql

11 https://marklogic.com — Since 2014, Marklogic has started an extra support for JSON documents and is considered as a multimodel store.

12 https://virtuoso.openlinksw.com

13 https://w3.org/TR/xquery-30/ 
and query processing and optimization. However, some applications have preferred JSON as an alternative to XML due to its relative compactness, simplicity and tight interaction with popular programming languages. In this respect, XML and JSON stores support different applications and use-cases. XML stores are usually used for organizing and maintaining a collection of XML files in content management applications such as health-care, science and digital libraries, whereas JSON stores are used by more interactive and dynamic Web applications.

\subsubsection{Graph stores}

The focus of the aforementioned data models is to store information about entities as either binary values, rows in wide-column tables, or documents. However, the increasing number of graph-oriented datasets, such as semantic Web [48], Web mining [49] and the interaction of proteins in biological systems [50,51], has motivated the emergence of graph stores for storing such datasets and querying of entity relationships in an efficient manner. These stores are based on the strong graph-theoretical foundation, where a graph consists of vertices representing entities and edges representing relationships between them.

Table 2.1 shows a list of graph structures that are not usually mutually exclusive. For example, property graphs are an amalgam of directed, labeled, attributed and multigraphs, which are widely adopted in practice. The popularity of property graphs is due to their flexibility to express other structures. For instance, by not using attributes in property graphs, $\mathrm{RDF}^{14}$ or semantic graphs are generated. An RDF graph is a set of RDF statements (or triples), where each of which represents a simple relationship between two entities.

Figure 2.1(d) illustrates an example of how Facebook uses a property graph for its

14 https://w3.org/RDF/ 
Table. 2.1. Various graph structures

\begin{tabular}{|c|c|}
\hline Graph structure & Description \\
\hline Undirected/directed graphs & All relationships in an undirected graph are symmetric. \\
\hline Labeled graphs & $\begin{array}{l}\text { Vertices and edges are tagged with scalar values (labels or types) that may represent } \\
\text { either their roles in different application domains or some attached metadata. }\end{array}$ \\
\hline Attributed graphs & $\begin{array}{l}\text { A variable list of attributes as } \mathrm{KV} \text { pairs are attached to vertices and edges, represent- } \\
\text { ing their properties. It is suitable for social networking sites that involve the social } \\
\text { interaction of individuals. }\end{array}$ \\
\hline Multigraphs & $\begin{array}{l}\text { Multiple edges (even with the same labels) between the same two vertices as well as } \\
\text { self-loops are allowed. }\end{array}$ \\
\hline Hypergraphs & $\begin{array}{l}\text { These graphs can represent } \mathrm{N} \text {-ary relationships through hyperedges which can con- } \\
\text { nect any number of vertices [52]. }\end{array}$ \\
\hline
\end{tabular}

social network, where users, physical locations, relationships (such as users' friendships) and actions (such as liking, writing a comment and checking-in to a location) are encoded with labeled vertices and labeled directed edges of a graph. Each vertex is uniquely identified by a vertex identifier and each edge by a triple of source and destination vertex identifiers plus the edge label. In addition, each edge label is accompanied with a set of properties (including the time property) as KV pairs. Note that all edges in Figure 2.1(d) are bidirectional (either symmetric such as FRIEND or asymmetric such as AUTHORS/AUTHORED_BY) except the edges whose label is COMMENT, because there is no need to traverse from the COMMENT to the CHECKIN vertex. Table 2.2 summarizes the characteristics of the discussed NoSQL data models.

\subsection{NoSQL data partitioning}

Data partitioning stands for the solutions whereby data in a database are split into some disjoint partitions and spread over different storage nodes. This can be achieved either horizontally (also called sharding) or vertically. Horizontal partitioning divides 
Table. 2.2. Comparison of the NoSQL data models

\begin{tabular}{|c|c|c|c|}
\hline & Fit scenario(s) & Strength(s) & Limitation(s) \\
\hline $\begin{array}{l}\text { Key- } \\
\text { value }\end{array}$ & $\begin{array}{l}\text { Domain entities are only ac- } \\
\text { cessed via a single Key; En- } \\
\text { tity caching; Entities are not } \\
\text { related. }\end{array}$ & $\begin{array}{l}\text { Scalable; Providing a very fast random ac- } \\
\text { cess via Key; The ease of data partition- } \\
\text { ing. }\end{array}$ & $\begin{array}{l}\text { The responsibility of the application pro- } \\
\text { grammer for the modeling of values; The } \\
\text { indexing and querying of entities is only } \\
\text { by their Keys. }\end{array}$ \\
\hline $\begin{array}{l}\text { Wide- } \\
\text { column }\end{array}$ & $\begin{array}{l}\text { Parallel processing of large ag- } \\
\text { gregated datasets. }\end{array}$ & $\begin{array}{l}\text { With regard to the workload, a hierarchy } \\
\text { of constructs, such as CFs, is designed } \\
\text { that in turn increase the performance of } \\
\text { queries; It is a suitable model for stor- } \\
\text { ing huge amounts of data as it can be ef- } \\
\text { ficiently partitioned horizontally by rows } \\
\text { and vertically by CFs. }\end{array}$ & $\begin{array}{l}\text { Providing limited ad-hoc querying as any } \\
\text { change in the application-specific access } \\
\text { patterns will impact the design to a large } \\
\text { degree; The predefined set of CFs makes } \\
\text { it difficult to use wide-column stores for } \\
\text { applications with evolving schemas. }\end{array}$ \\
\hline Document & $\begin{array}{l}\text { Data can be easily interpreted } \\
\text { as documents; Data are con- } \\
\text { stantly evolving. }\end{array}$ & $\begin{array}{l}\text { A rich data model to store data with arbi- } \\
\text { trary complexity such as nested constructs, } \\
\text { arrays and scalar values; Each component } \\
\text { of a document can be accessed via sec- } \\
\text { ondary indices. }\end{array}$ & $\begin{array}{l}\text { There is no standard API or query lan- } \\
\text { guages. }\end{array}$ \\
\hline Graph & $\begin{array}{l}\text { There is a need to traverse } \\
\text { several levels of relationships } \\
\text { among intensely related data. }\end{array}$ & $\begin{array}{l}\text { Providing fast and simple querying of } \\
\text { linked datasets; Easy mapping of entity- } \\
\text { relationship diagrams. }\end{array}$ & $\begin{array}{l}\text { There is no standard API or query lan- } \\
\text { guages; Partitioning of large graphs re- } \\
\text { duces the performance due to the high } \\
\text { amount of inter-node communications. }\end{array}$ \\
\hline
\end{tabular}

the data at the row-level, such as rows in a wide-column table or documents in a collection, into some disjoint partitions or shards. Vertical partitioning divides the data that are commonly accessed together, such as the columns of a CF, into some disjoint partitions.

Data partitioning has some advantages. First, it improves the scalability of the system as it tackles the situations when large volumes of data and high request rates overwhelm the storage and processing capacity of any single server. Second, it improves the performance of the system by increasing the degree of parallel processing on multiple partitions. Finally, it works well for geographically dispersed data, where reads and writes are primarily within one geographic location. Note that partitioning strategies are usually supplemented with replication mechanisms to avoid any single point of failure. Sharding can be either key-oriented as data lookup is just based 
on some shard keys or traversal-oriented as data lookup is based on analyzing the relationships between entities. Hence, data models have a significant effect on selecting the strategy of partitioning. In this section, some of the well-known sharding strategies are explained.

\subsubsection{Key-oriented static partitioning}

This kind of sharding aims at balancing the utilization of static space, while dynamic data and workloads are entirely disregarded. Some of the corresponding strategies are as follows.

- Range-based, thereby data items are clustered according to the contiguous intervals of shard keys, such as the range of a unique identifier. As an advantage, range queries on short intervals are handled efficiently as they involve communicating with only a single partition or a few partitions. In other words, the number of multipartition requests is decreased. This kind of partitioning is very useful for applications such as data warehousing [53], Web servers [54] and online games [55], which involve distributed order-preserving data structures. However, there are some drawbacks. First, popular keys, such as the letter 'E', cause the workload to be skewed so that a single or a few partitions receive a larger fraction of requests than the others. Second, the non-uniform distribution of inserting entities' keys, causes data workload to be skewed so that storage nodes become unbalanced. Finally, it needs to maintain a central lookup table or directory to store the mapping of partitions to the storage nodes. The directory service may decrease the performance and scalability as a round-trip delay is added to the critical data access path. This strategy is used by some NoSQL stores such as Google BigTable, Apache HBase, MongoDB, Yahoo Pnuts [56] and IBM Spinnaker [57]. 
- Simple hashing, thereby entities' keys are randomly hashed to their hosting nodes via simple hashing schemes such as the modulo hashing where:

\section{HostingNodeNumber $=$ Key modulo NumberOfNodes}

Relying on hash functions results in efficient data lookups as they are performed locally [58]. However, in simple hashing, adding or removing a node necessitates redistributing a lot of entities as the hash of keys severely reshuffles. This incurs a high cost for regularly expanding datasets. Furthermore, there is poor data locality and the number of multipartition requests is increased as logically related entities are randomly spread over different nodes.

- Consistent hashing, where the scope of a hash function is considered as a "ring" and both nodes' IDs, such as IP addresses, and entities' keys are randomly hashed to the ring's positions $[59,60]$. The hosting node of an entity is the first node encountered when walking clockwise from the entity's position on the ring. Suppose for a given node hashed at position $p$, the positions of its immediate predecessor and successor are specified by $p$.predecessor and $p$.successor respectively. Therefore, a node with position $p$ is responsible for an individual set of entities whose keys are hashed to an arc or partition of the ring between $p$.predecessor and $p$. When a new node joins and hashed at position $q$, the old arc corresponding to its immediate successor is split into two new adjacent arcs between $q$.predecessor and $q$, as well as $q$ and $q$.successor. On the other hand, by leaving or failing a node, the old arcs corresponding to the node and its successor are merged with each other.

As a result, on the contrary to the simple hashing, the addition or removal of a node in consistent hashing just incurs redistributing $\mathcal{O}(1 / N)$ fraction of entities that are hosted on the node's successor, where $N$ is the number of existing nodes. This means consistent hashing scales much better than simple 
hashing. However, there are some drawbacks. First, it still suffers from poor data locality. Second, for a uniform distribution of entities over the keyspace, there is an $\mathcal{O}(\log N)$ imbalance factor between $N$ nodes, in terms of stored entities and workload [61]. Third, the performance heterogeneity of nodes is not taken into account as it may result in data and workload imbalance. Finally, by joining or leaving a node, data redistribution may overwhelm the load capacity of the node's successor.

Most of these issues can be addressed by using virtual nodes or shards [61,62], thereby multiple non-contiguous positions in the hash ring can be assigned to a physical node. The number of positions or virtual nodes corresponds to the capacity of the node. In more detail, the stronger physical server will split into more virtual nodes. However, some systems (e.g., Apache Cassandra) put a limit on the number of virtual nodes as they have several side-effects [63] such as increased agitation due to the failure of a physical node and increasing state maintenance. Some NoSQL stores, such as Apache Cassandra, Amazon Dynamo [58], Riak KV ${ }^{15}$ and Microsoft Trinity [64], use this strategy. Variants of consistent hashing are commonly used in DHTs $[61,65,66]$ where data are stored and looked up in a totally decentralized manner.

\subsubsection{Key-oriented workload-aware partitioning}

In the above sharding strategies, dynamic query and data workloads are not taken into account. For example, the static hash function used in the consistent hashing strategy cannot tackle hotspots (i.e., nodes that are heavily loaded) caused by the non-uniform query distributions of real-world workloads. A workload-aware partitioner identifies hotspots and then dynamically adjusts the load distribution $[67,68]$. This dynamic adjustment must guarantee there is no ping-pong phenomena [69] or $\overline{15}$ https://basho.com/products/riak-kv/ 
continuous relocation of a data item across nodes. One such partitioning strategy is the migration of virtual nodes, where each physical node can accommodate several virtual nodes according to both its capacity and query distribution [61,70-72]. It alleviates detected hotspots by reassigning some of their virtual nodes to light nodes. A set of centralized directory nodes is maintained by the system. They periodically receive the load information of different nodes and based on that assign virtual nodes to physical servers.

\subsubsection{Traversal-oriented partitioning}

Balanced $k$-way graph partitioning divides the graph into $k$ disjoint and balanced partitions and minimizes the cut size. This is a well-known NP-hard problem, where computational load balance, in order to maximize parallelism, and data access locality, in order to minimize inter-node communication, are two conflicting issues [73]. This results in lots of heuristic partitioning methods for graph datasets, which can be classified as two orthogonal categories as follows.

- Vertex-centric vs. edge-centric partitioning, where the vertex-centric partitioners assign each source vertex along with its incident edges into the same partition, which in turn increases locality. However, the corresponding destination vertices may be assigned to different partitions which results in cutting their in-between edges or edge-cuts. These partitioners aim at performing a balanced distribution of vertices across nodes, as well as minimizing the number of edgecuts. Although vertex-centric partitioners promote locality, they may severely impact the computational load balance for power-law graphs, where a small fraction of vertices have extremely high degrees in proportion to others [74]. In other words, by grouping all edges of high-degree vertices together, a subset of nodes are overloaded. On the other hand, edge-centric methods tend to assign 
the edges incident to a particular vertex into different partitions. However, the endpoint vertices of an edge are replicated in the same node as the edge places. These partitioners aim at performing a balanced distribution of edges across nodes, as well as minimizing the number of replicas. Although edge-centric partitioners alleviate the computational load imbalance of high-degree vertices, they often incur higher communication and synchronization cost through the poor locality [75].

- Offline vs. online partitioning, where offline or non-streaming partitioners, such as METIS [76], require accessing to the whole graph dataset in order to perform preprocessing prior to partitioning. However, they scale poorly against very large graphs due to their heavy usage of memory and high computational cost, which in turn impacts the performance of online (non-analytical) query processing. This partitioning approach has been later improved through parallelization techniques, such as ParMETIS [77]. These parallel strategies yet suffer from the need for a global view of the graph that reduces their scalability.

Since scaling offline approaches for large graphs is difficult, online or streaming approaches have been introduced which continually update the partitioning as new changes are streamed into the system. More precisely, they partition the incoming vertices (for vertex-centric partitioning) or edges (for edge-centric partitioning) one at a time based on the local knowledge of the input graph such as the current properties of streamed elements and the information of previously partitioned ones. These streaming approaches are one-pass since after assigning a vertex or edge to a partition, no reassignment is performed. Due to the online nature of these approaches, lightweight heuristics, such as Fennel [78], are used to decide where to assign incoming elements. However, as graph elements are assigned once, new streamed elements of a graph may deteriorate its previous 
partitioning. Hence, there are several extensions $[79,80]$ of the streaming approach, where graphs are partitioned in several passes or iterations. But the quality of partitions is still dependent on the ordering of streamed elements as there may not be enough local knowledge of the input graph.

\subsection{Summary}

Although data partitioning is regarded in distributed database systems as a quintessential technology, current NoSQL stores suffer from several data partitioning limitations. First, the number of multi-partition requests (i.e., the processing of a query requires contacting several partitions) should be decreased. This reduces the amount of data transferred over the network during query processing. Second, the distribution of processing and storage load should be uniform with regard to the capacity of nodes (e.g., processing power, disk speed and network capabilities). Third, the amount of data transferred during repartitioning events, such as a node addition or removal, should be reduced. Finally, the storage, retrieval and manipulation of mapping between partitions and storage nodes should be efficient. As data modeling and partitioning are two non-orthogonal principles, Section 3 elaborates on how a workload-driven NoSQL data modeling can result in efficient data partitioning. 


\section{Chapter 3}

\section{Partitioning of Aggregate-Oriented}

\section{Databases}

As presented in section 1.4, the main deliverable of this thesis is a framework for the schema design and partitioning of NoSQL databases. As part of the framework, this chapter focuses on our method for the workload-driven modeling and partitioning of aggregate-oriented NoSQL databases. In more detail, the lack of standard processes for designing NoSQL database schemas has led to the proposal of several recent methods. However, they provide various conceptual modeling notations and mainly target a particular NoSQL data model that cause extra effort for designers when switching from one data model to another. In addition, by providing just a set of guidelines and heuristics for the design process, many methods have to be applied manually which is an error-prone and time-consuming process. To deal with these limitations, we present a method for designing key-value, wide-column, and document NoSQL database schemas from the same conceptual model. It first generates a generic NoSQL logical schema from the conceptual model and workload of the system. Then it converts the generic schema to the schemas of targeted NoSQL data models regarding

their important features and design trade-offs between the read query performance and storage overhead or consistency maintenance. 


\begin{tabular}{|c|c|c|c|c|c|c|}
\hline Customer & \multirow{4}{*}{$\underset{\text { requests }}{\text { requests }}$} & Order & \multirow{4}{*}{ consists_of } & & \multirow{4}{*}{ references } & Product \\
\hline id: INT & & id: INT & & Item & & id: INT \\
\hline name: TEXT & & date: TEXT & & id: INT & & name: TEXT \\
\hline contacts: $\{$ TEXT $\}$ & & totalPrice: INT & & quantity: INT & & price: INT \\
\hline
\end{tabular}

Fig. 3.1. Conceptual schema of a sample e-commerce platform

\subsection{Concepts and formalization}

This section starts by illustrating various modeling concepts through a running example. It then elaborates the aforementioned aggregate-oriented NoSQL data models (see section 2.1) by introducing their popular representatives, i.e., Oracle NoSQL [81], Cassandra [82] and MongoDB [83], followed by formalizing the models.

\subsubsection{Running example}

Figure 3.1 shows the conceptual schema of a sample E-commerce system, that is represented as an entity graph (see Section 3.3.1). This is similar to the one used in existing online retailers, such as Amazon. As it shows, this system manages four entity types: Customer, Order, Item and Product. We assume that the corresponding entities are accessed in a workload containing the following query patterns:

- $\mathbf{q}_{1}$. Given a customer $i d$, return the corresponding products after a given date.

- $\mathbf{q}_{2}$. Given an order $i d$, return the corresponding products ordered by name in ascending order.

- $\mathbf{q}_{3}$. Given an order $i d$, return the corresponding customer and products ordered by name in ascending order.

In our subsequent examples, Order is considered as a HW entity type as its corresponding entities receive lots of write operations (i.e., insert, delete or update). Hence, the denormalization of HW entities might impact write operations due to consistency 
maintenance overhead. In addition, Product is considered as an HU entity type as it is used by a majority of query patterns in the workload (i.e., all the three patterns in the above example). Therefore, the denormalization of HU entities might increase the cost of storage due to data redundancy. Note that assuming a workload as a set of pairs of query patterns and their corresponding frequencies, being an HU entity type does not necessarily mean that the corresponding entities receive lots of read operations. Regarding the above definitions, designers are responsible to specify HW entity types as it needs understanding their meanings. On the other hand, HU entity types are automatically identified by scanning all query patterns in the workload.

\subsubsection{KV schema formalization}

In advanced KV stores, such as Oracle NoSQL, the Key part is structured as the combination of a major key and an optional minor key separated by the hyphen ('-'). A composite key includes a sequence of components, where the components are separated by the forward-slash ('/9). The Value part can be either schema-less as a byte array or schema-full using Table API [84]. In a distributed system, KV pairs are spread evenly across partitions through hashing their major keys. Applications can take advantage of major keys to achieve data locality for all KV pairs that share the same major key. More precisely, the proper design of major keys results in applying a single atomic operation with ACID guarantees on multiple KV pairs that share the same major key, and the even distribution of KV pairs across partitions. Note that KV pairs that share the same major key are sorted in ascending order of the value of the first component in the sequence of minor key components. An Oracle NoSQL database schema is a set of KV schemas, defined as follows.

Definition 3.1. A KV schema is a sequence of components divided into the major key, the minor key and the value part, denoted by $S\left(c_{1}, c_{2}, \ldots, c_{n}\right)$, where $S$ is the schema name, and each component $c_{i}$ is associated with a type $t_{i}$ and a role $r_{i}$, 


\begin{tabular}{|lll|} 
S1 & & \\
\hline orderID & TEXT & Major \\
prodcuName & TEXT & Minor \\
itemID & TEXT & Minor \\
productPrice & FLOAT & $V$ \\
customerName & TEXT & $V$ \\
customerContacts & $\{$ TEXT $\}$ & $\mathrm{V}$ \\
\hline
\end{tabular}

(a)

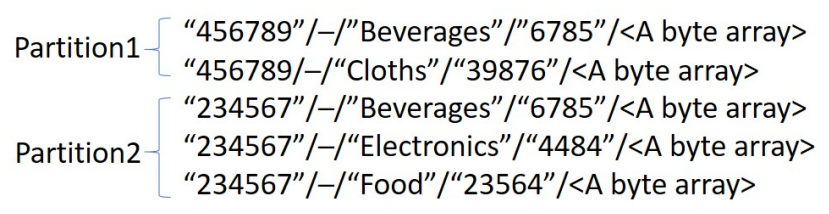

(b)

Fig. 3.2. (a) a KV logical schema that satisfies both the queries $q_{2}$ and $q_{3}$ in our running example; and (b) some instances of the schema

$t_{i}$ is either a primitive INT, FLOAT, TEXT or DATE, or a collection of primitives constructed with a set $(\{\})$, a list ([]), or a map as a container of key-value pairs, and $r_{i}$ specifies that the component is a part of either the major key (MAJOR), the minor key (MINOR), or values (V). There are at least one MAJOR and one V components.

For example, Figure 3.2(a) depicts a KV logical schema named $S_{1}$, where the design of the Key and Value parts satisfies both the queries $q_{2}$ and $q_{3}$ in our running example; and Figure 3.2(b) shows some instances of the schema where orderID, as a single component of the major key, along with (productName, itemID), as the sequence of minor key components, ensure the uniqueness of each pair. The major key ensures that all items associated with an order are hosted in the same node. The selection of productName as the first component in the sequence of minor key components is just to ensure that all products associated with an order are sorted based on their names. As shown in the figure, the first instance is composed of "456789"/-/"Beverages"/"6785" as the Key, and a byte array of the associated product price, as well as the name and contacts of the associated customer as the Value.

Oracle NoSQL offers simple atomic operations for accessing and modifying individual KV pairs, namely put(key, value) for the addition or modification of a pair and get(Key) for the retrieval of a value corresponding to the key. In addition, it offers an 
atomic operation multiGet(parentKey) for the retrieval of multiple sorted KV pairs having the same parentKey. Note that parentKey is a subsequence of the major key components. Moreover, it provides a put operation for atomic and efficient execution of multiple simple put operations (assuming that the same major key is shared by all the keys used in these operations).

\subsubsection{Wide-column schema formalization}

In wide-column stores, such as Cassandra, a CF consists of a set of partitions, where each partition is a container of rows that are accessed together. There is a mandatory partition key for each partition. In addition, there might be a unique clustering key for each row in a partition. A composite key is a sequence of columns. A primary key that uniquely identifies a row in a CF is the combination of a partition key and a clustering key. In order to increase scalability, the partition key is utilized to distribute the partitions of a CF into different nodes. As such, Cassandra hashes the value of the partition key in order to determine which node is responsible for storing the corresponding partition. On the other hand, rows with the same partition key are stored together to increase the locality of accesses. The clustering key allows the physical ordering of rows within each partition. When there is no clustering key in a CF, each partition of the $\mathrm{CF}$ contains a single row whose primary key is equivalent to the partition key. On the other hand, when there is a clustering key in a $\mathrm{CF}$, each partition of the $\mathrm{CF}$ contains multiple rows whose primary keys share the same partition key and have different clustering keys. The clustering key of a CF is also used to arrange the rows of each partition in the CF in ascending (default) or descending order A Cassandra NoSQL database schema is a set of CF schemas, defined as follows.

Definition 3.2. A CF schema is a sequence of components divided into the partition key, the clustering key and ordinary components, denoted by $S\left(c_{1}, c_{2}, \ldots, c_{n}\right)$, 


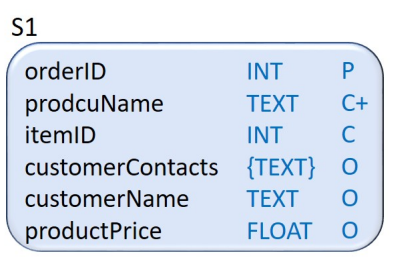

(a)

\begin{tabular}{|c|c|c|c|c|c|c|}
\hline & orderID & productName & itemID & customerContacts & customerName & productPrice \\
\hline \multirow{3}{*}{ Partition1 } & 456789 & "Beverages" & 4484 & $\{\ldots\}$ & “John" & $\ldots \$$ \\
\hline & 456789 & "Cloths" & 39876 & $\{\ldots\}$ & "John" & $\ldots \$$ \\
\hline & 234567 & "Beverages" & 4484 & $\{\ldots\}$ & "Mike" & $\ldots \$$ \\
\hline \multirow[t]{2}{*}{ Partition2 } & 234567 & "Electronics" & 6785 & $\{\ldots\}$ & "Mike" & $\ldots \$$ \\
\hline & 234567 & "Food" & 23564 & $\{\ldots\}$ & "Mike" & $\ldots \$$ \\
\hline
\end{tabular}

(b)

Fig. 3.3. (a) A CF logical schema that satisfies both the queries $q_{2}$ and $q_{3}$ in our running example; and (b) the corresponding instance

where $S$ is the schema name, and each component $c_{i}$ corresponds to a type $t_{i}$ and a role $r_{i}, t_{i}$ is either a primitive INT, FLOAT, TEXT or DATE, or a collection of constructed types using set " \{\} ", list "[ ]", and map " $<>$ " constructors on primitives; and $r_{i}$ is either a component of the Partition key specified by $\mathrm{P}$, the Clustering key specified by $\mathrm{C}[+\mid-]$ for ascending or descending order, or an Ordinary column specified by $\mathrm{O}$. There are at least one $\mathrm{P}$ and one $\mathrm{O}$ components.

For example, Figure 3.3(a) and (b) depict a CF logical schema named $S_{1}$ in order to satisfy both the queries $q_{2}$ and $q_{3}$ in our running example; and the corresponding instance, respectively. According to the aforementioned query requirements, this design allows each order and its associated items to be vertically partitioned, while the information of all orders are horizontally partitioned. Here, orderID, as a single component of the partition key, along with (productName, itemID), as the sequence of clustering key components, make a primary key that ensures row uniqueness. The selection of orderID as the partition key ensures that all items associated with an order are hosted in the same node. Also, the selection of productName as a component of the clustering key is just to ensure sorting the items belonging to each order. This design of partition and clustering keys allows an efficient retrieval of information in queries $q_{2}$ and $q_{3}$. As Cassandra supports complex data types, such as user-defined data types, maps, lists and sets, we use a set type for the contacts property. 


\subsubsection{Document schema formalization}

In document stores, such as MongoDB, documents are stored in BSON format [85], that is a JSON-like structure. It allows the structure of a collection to be defined via a JSON schema [86]. MongoDB supports (ascending/descending) indices on properties of documents. The ordering of the index entries supports efficient equality matches and range-based query operations. A MongoDB NoSQL database schema is a set of document collection schemas, defined as follows.

Definition 3.3. A document collection schema is a set of hierarchical properties, denoted by $S\left(p_{1}, p_{2}, \ldots, p_{n}\right)$, where $S$ is the schema name, and each property $p_{i}$ corresponds to a type $t_{i}$ and a role $r_{i}, t_{i}$ is either a primitive INT, FLOAT, TEXT or DATE, an embedded document specified by D, a reference to a document specified by ${ }^{*} n$, where $n$ is the name of the document collection, or a collection of constructed type using set " \{\} ", list "[ ]", and map " $<>$ " constructors on either primitives, documents or references to documents; and $r_{i}$ is either an indexed property specified by $\mathrm{I}[+\mid-]$ for ascending or descending order, or not indexed specified by NULL. There is at least one (ascending or descending) index over properties.

For example, Figure 3.4(a) depicts a document collection logical schema named $S 1$ that embeds orders and associated items in our running example, where customerID and orderDate are indexed. This allows an efficient retrieval of information in query $q_{1}$, where join operations are avoided. More precisely, by retrieving a customer, its related orders and associated items are also retrieved, which avoids performing joins with the data of other collections. On the other hand, as Figure 3.4(b) shows, we may prefer to join customers, orders and items rather than embedding (see Section 3.3.7 for more detail). 


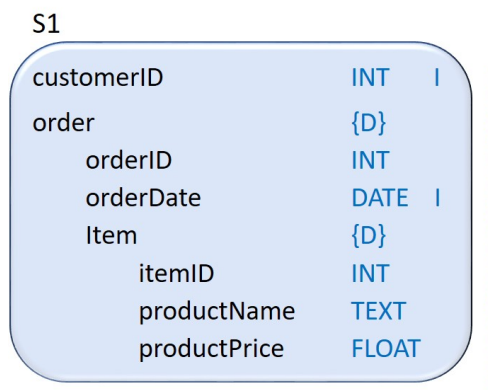

(a)

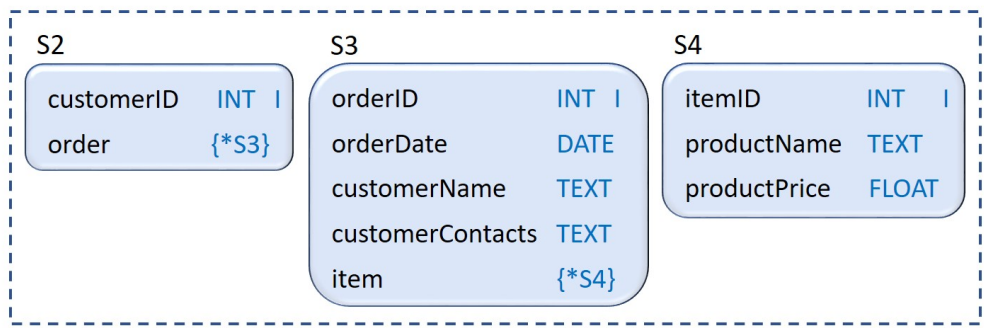

(b)

Fig. 3.4. Document collection schemas that satisfy $q_{1}$ in our running example, by either (a) embedded documents or (b) joined documents

\subsection{Related works}

In this section, we briefly describe the existing works that address the database schema design of aggregate-oriented NoSQL stores. Table 3.1 summarizes each one of these works. Li [87] proposes one of the very first work on the schema design of NoSQL databases. It provides a set of heuristics, whereby the relational schema is mapped into the HBase database schema [94]. This method, however, is workload-agnostic and does not necessarily generate database schemas that can efficiently implement any specific workload. Chebotko et al. [89] propose a workload-driven design method for transforming an ER model into a database schema in Cassandra. This mapping is based on a set of rules regarding the application workflow describing query or data access patterns. As this mapping generates a CF for each query, it might end up with a high level of denormalization that, in turn, increases the performance of read queries. However, as the cost of consistency maintenance is increased, the performance of write queries might be decreased.

The above challenge is because of using the same plan for mapping CFs. Therefore, Mior et al. [91] propose a tool, called NoSE, which takes into account all alternative plans to generate a wide-column database schema. It maps a given ER model along 
Table 3.1. Database schema design processes for aggregate-oriented NoSQL stores

\begin{tabular}{lcccccc}
\hline & $\begin{array}{c}\text { Conceptual } \\
\text { schema }\end{array}$ & Logical schema & $\begin{array}{c}\text { Supported } \\
\text { data models }\end{array}$ & Queries & Workload information & $\begin{array}{c}\text { Process } \\
\text { automation }\end{array}$ \\
\hline Li [87] & $x$ & Relational & W & $x$ & $x$ & $x$ \\
\hline Atzeni et al. [88] & UML & NoAM & K,W,D & $x$ & $x$ & $\checkmark$ \\
\hline Chebotko et al. [89] & ER & In-house notation for CFs & W & ERQL [90] & $x$ & $\checkmark$ \\
\hline Mior et al. [91] & ER & $x$ & W & SQL-based & $\begin{array}{c}\text { Estimated query } \\
\text { frequencies \& data volume }\end{array}$ \\
\hline de Lima and Mello [92] & EER & $\begin{array}{c}\text { In-house notation } \\
\text { for documents }\end{array}$ & D & XML-based [93] frequencies \& data volume & $\checkmark$ \\
\hline Our method & Entity graph & Query Path Graph & K,W,D & SQL-based & HW \& HU entity types & $\checkmark$ \\
\hline
\end{tabular}

Key-value (K), Wide-column (W), Document (D)

with the workload information of estimated query frequencies and volume of data into all candidate plans and selects the one offering the best performance through a BIP approach [95]. de Lima and Mello [92] propose a similar design method, but focusing on MongoDB. It converts an EER model into a logical model to represent document collections. This transformation is based on a set of rules regarding the workload information of estimated query frequencies and volume of data, in order to avoid data redundancy.

Atzeni et al. [88] propose a generic design method for aggregate-oriented NoSQL stores. It provides a number of heuristics in order to map a given UML model into a logical model called NoAM, built by the authors themselves. The mapping strategy is based on the concept of "nested data unit". In our running example, Customer and Order might build a nested data unit. Each unit is mapped into a NoAM block that is an abstraction of NoSQL constructs such as document collections or CFs. Since their mapping strategy is not workload-driven, it may be problematic in either identifying important features of the target data model, such as the major and minor keys of a KV schema, or performing physical optimizations. In addition, the naive one-to-one conversion of NoAM blocks into NoSQL constructs may impact the quality 


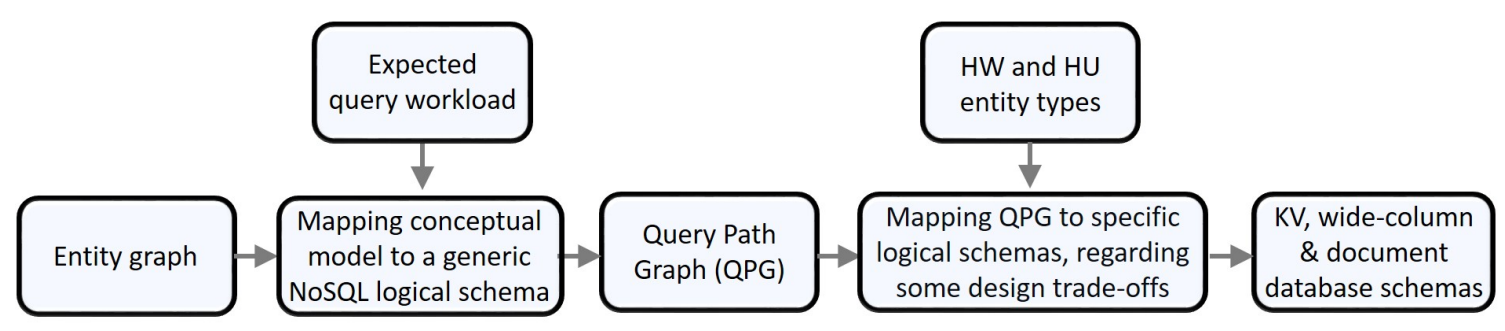

Fig. 3.5. An overview of our method for designing NoSQL database schemas

of design. For example, according to the aforementioned methods, despite a single CF that is usually designed per query $[87,89,91]$, a single document collection may satisfy multiple queries [92]. However, the NoAM generic model just allows creating the same number of CFs and document collections as the number of queries.

To summarize, as shown in Table 3.1, the majority of methods target a particular NoSQL data model. On the other hand, the NoAM generic method [88] does not take into account the specific features of different NoSQL data models that, in turn, prevents the automation of its design process and impacts the quality of generated database schema. Our generally applicable method simply characterizes workloads based on HW and HU entity types. Here, designers can tailor or customize database schema generation based on HW and HU entity types, whereby the denormalization of the corresponding entities is controlled. In more detail, they can activate the generation based on HW entities, which means trading read query performance for consistency maintenance. In addition, activating the generation based on HU entities, means trading read query performance for storage overhead.

\subsection{Solution description}

Figure 3.5 shows how our method is used to design aggregate-oriented NoSQL database schemas, which is explained in successive sections in more detail. 


\subsubsection{Conceptual schema}

Designing a NoSQL database schema starts with a conceptual schema that defines a concise overview of user data requirements and provides detailed descriptions of the entity types, associations and constraints [96]. We represent this schema as an entity graph, defined as follows.

Definition 3.4. An entity graph is a labeled directed multigraph, denoted as $C$ $=\left(N_{C}, E_{C}, L_{C}\right)$, where $N_{C}$ is a set of nodes corresponding to entity types, $E_{C} \subseteq N_{C}$ $\times N_{C}$ is a set of edges corresponding to associations between entity types, and $L_{C}$ is a set of labels corresponding to associations. A node is a set of attributes, denoted by $e\left(a_{1}, a_{2}, \ldots, a_{n}\right)$, where $e$ is an entity type name, and each attribute $a_{i}$ is associated with a type $t_{i}$ and a role $r_{i}, t_{i}$ is either a primitive INT, FLOAT, TEXT or DATE, or a collection of primitives constructed with a set $(\{\})$, a list $([])$, or a map $(<>)$ constructor, and $r_{i}$ specifies that the attribute is either a key $(\mathrm{K})$ or not. There is at least one key attribute. An edge $\left\langle e_{i}, e_{j}\right\rangle$ with a single or double arrow on $e_{j}$ side represents the cardinality of association toward that side, meaning either "one" or "many" respectively.

For example, as the entity graph in Figure 3.1 shows, the Customer entity type has three attributes: $i d$, name and contacts, where $i d$ is a key. In addition, each customer is associated with many orders.

\subsubsection{Query modeling}

Given an entity graph $C$, we represent each query as an acyclic subgraph or path of $C$, starting from an entry or access point of the query. This is because the existence of a cycle in a query path challenges our mapping strategies when data are denormalized. In more detail, this recursive nesting may result in infinite loops (see line 13 in Algorithm 2, line 16 in Algorithm 4 and line 13 in Algorithm 6, due to the existence 


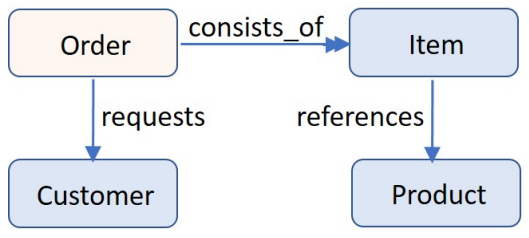

(a)

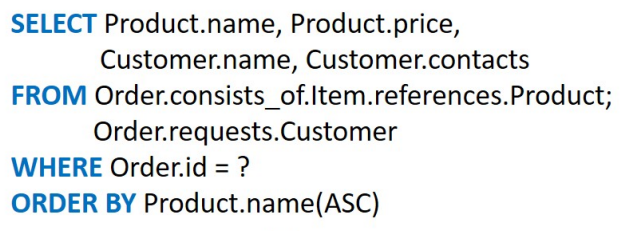

(b)

Fig. 3.6. (a) the query path of $q_{3}$; (b) a SQL-based syntax of $q_{3}$

of a directed cycle). Note that due to the simplicity of queries in aggregate oriented NoSQL stores, query paths are mostly linear [10].

Similar to GMAP [97] that proposes a SQL-based query language independent of database schemas, we use the following simple grammar for read queries over an entity graph:

\section{SELECT attributes FROM query path}

WHERE attribute $\{=|<|<=|>|>=\}$ value

[AND ...]

[ORDER BY attribute[(ASC)]|[(DESC)] [, ...]]

where the FROM clause specifies query path, that determines how the entities accessed by the query create a nested data unit. The SELECT clause specifies projection attributes of one or more entity types on the path; regarding one or more equality and/or inequality conditions over the entity types, specified by the WHERE clause. The results can also be ordered by some attributes of the entity types, specified by the ORDER BY clause. For example, Figures 3.6(a) and (b) show a query path of $q_{3}$ in our running example along with the corresponding SQL-based syntax, respectively. As aforementioned, as long as there is no cycle in the query path extracted from a query, it can be exploited in our mapping strategy to denormalize data. Hence, even complex query templates (with no cycle) have no impact on our mapping strategy. However, we did not include them due to space limitations. 


\subsubsection{Query path graph}

A conceptual schema that just specifies what entity types the system is composed of and how they are associated is not enough to design a NoSQL database schema, as previously mentioned and pointed out by other authors [89,91,92]. As a complement, we use the corresponding workload to determine how entities are accessed and by what queries. Hence, we combine such information in a QPG, defined as follows.

Definition 3.5. Given a workload with a set of query patterns $Q=\left\{q_{1} \ldots q_{n}\right\}$ over an entity graph $C$, the corresponding QPG is denoted as $G=\left(N_{G}, E_{G}, L_{G}\right)$, where $N_{G} \subseteq N_{C}$ is a set of entity types accessed by $Q ; E_{G} \subseteq E_{C}$ is a set of edges that are traversed by $Q$; and $L_{G} \subseteq L_{C} \cup\{1 . . n\}$. Here, a node $e_{i} \in N_{G}$ is an access point of $q_{m} \in Q$, iff there is at least one output edge $\left\langle e_{i}, e_{j}\right\rangle \in E_{G}$, labelled with $m$, and there is no input edge $\left\langle e_{k}, e_{i}\right\rangle \in E_{G}$, labelled with $m$.

Intuitively, a QPG is a subgraph of the entity graph, that is composed of the paths of all queries in a corresponding workload and is labeled with query numbers. In addition, each QPG node may be an access point of one or more queries. For example, Figures 3.7(a) and (b) show the paths of queries in the workload of the running example together with the corresponding QPG respectively. As shown in Figure 3.7(b), the double arrow of the edge $\langle$ Order, Item $\rangle$ via the association consists_of implies building a nested data unit of each order and multiple associated items. In addition, Order has some output edges whose labels contain 2 or 3 , but it has no input edge with any of these query numbers. This means that Order is the access point of $q_{2}$ and $q_{3}$. This also implies using the characteristics of queries $q_{2}$ and $q_{3}$ for the mapping of Order to specific NoSQL constructs, such as document collections. This may also lead to an optimization, as instead of mapping Order into separate NoSQL constructs for $q_{2}$ and $q_{3}$, it might be mapped into a common construct satisfying both the queries. Similarly, Customer is the access point of $q_{1}$. 


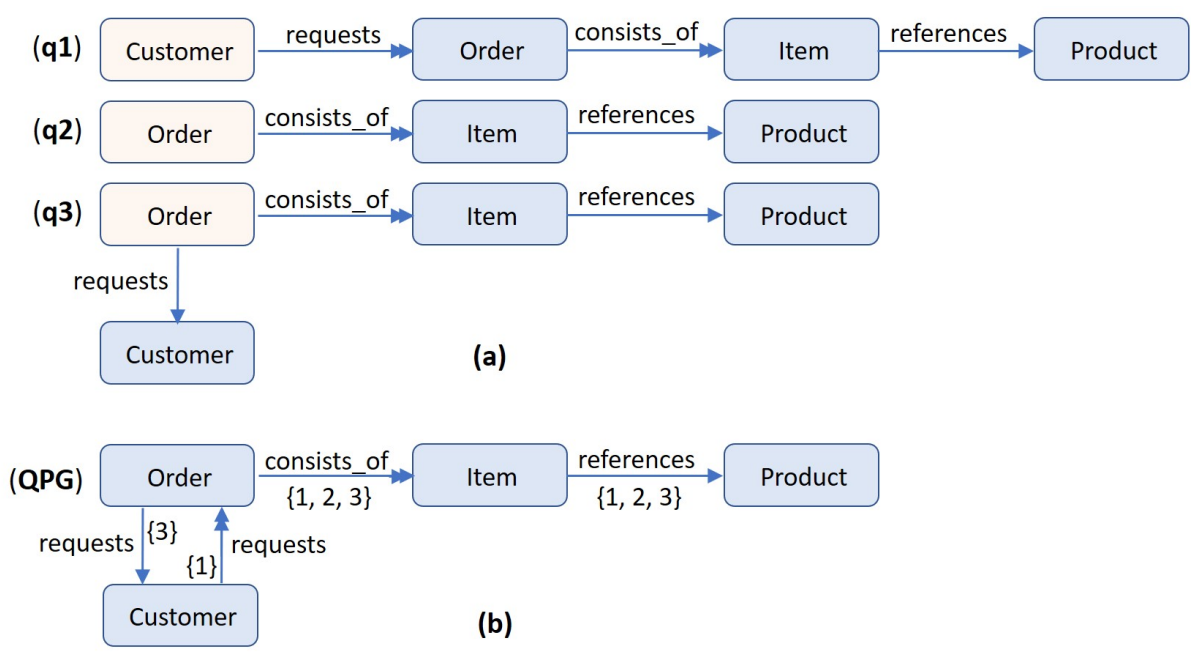

Fig. 3.7. (a) the paths of queries in the workload of the running example; (b) the corresponding QPG

\subsubsection{QPG specifications}

For a better understanding of subsequent algorithms for mapping a QPG into the constructs of different NoSQL database schemas, this section describes all the functions required to access a QPG. According to Definition 3.5, a QPG is represented with three specifications for nodes, edges and queries. Tables 3.2(a), (b) and (c) depict the three specifications, regarding the QPG in our running example shown in Figure 3.7(b). According to the specifications, Table 3.3 lists all the functions required to access a QPG, along with their descriptions.

\subsubsection{Mapping QPG to KV schemas}

This section describes the process for automatically mapping a given QPG into a combination of KV schemas. This mapping process is described in Algorithms 1 and 2 in detail. For instance, let us dig into the mapping of query $q_{2}$ in our running example. A KV schema $S 2$ is initialized for $q_{2}$ with two empty sets for key and 
(a)

\begin{tabular}{|c|c|c|c|c|c|}
\hline $\begin{array}{c}\text { QPG } \\
\text { node }\end{array}$ & Attributes & $\begin{array}{c}\text { Key } \\
\text { attributes }\end{array}$ & $\begin{array}{c}\text { Access } \\
\text { point? }\end{array}$ & Label & $\begin{array}{c}\text { Out } \\
\text { neighbors }\end{array}$ \\
\hline Customer & $\begin{array}{c}\text { id/INT, name/TEXT, } \\
\text { contacts/\{TEXT\} }\end{array}$ & id & Yes & 1 & Order \\
\hline Order & id/INT, date/TEXT, totalPrice/INT & id & Yes & 2,3 & Customer, Item \\
\hline Item & id/INT, quantity/INT & id & No & - & - \\
\hline Product & id/INT, name/TEXT, price/INT & id & No & - & - \\
\hline
\end{tabular}

(b)

\begin{tabular}{|c|c|c|}
\hline QPG edge & Label & Cardinality \\
\hline <Customer, Order> & 1, requests & $*$ \\
\hline <Order, Item> & $1,2,3$, consists_of & $*$ \\
\hline <Order, Customer $>$ & 3, requests & 1 \\
\hline <item, Product $>$ & $1,2,3$, references & 1 \\
\hline
\end{tabular}

(c)

\begin{tabular}{|c|c|c|c|c|}
\hline Query\# & $\begin{array}{c}\text { Equality } \\
\text { attributes }\end{array}$ & $\begin{array}{c}\text { Inequality } \\
\text { attributes }\end{array}$ & $\begin{array}{c}\text { Ordering } \\
\text { attributes }\end{array}$ & $\begin{array}{c}\text { Projection } \\
\text { attributes }\end{array}$ \\
\hline 1 & Customer.id & Order.date & - & Product.name, Product.price \\
\hline 2 & Order.id & - & Product.name(ASC) & Product.name, Product.price \\
\hline 3 & Order.id & - & Product.name(ASC) & $\begin{array}{c}\text { Product.name, Product.price, } \\
\text { Customer.name, Customer.contacts }\end{array}$ \\
\hline
\end{tabular}

Table 3.2. (a) specification of QPG nodes; (b) specification of QPG edges; (c) specification of QPG queries

value components. By traversing the corresponding query path (see Figure 3.7(a)), the schema $S 2$ is evolved as follows: the node Order is firstly processed, whereby attribute Order.id, which is an equality search attribute of $q_{2}$, is chosen as a MAJOR component. As a result, data are horizontally partitioned based on the Order.id, whereby the data required for a given Order.id are stored in the same partition. The node Item is subsequently processed, whereby attribute Item.id, that is a key attribute of Item, is chosen as a MINOR component. This is because there might be many Items associated with the same Order, that need to be uniquely identified. Finally, the node Product is processed, whereby the attribute Product.price that is a projection attribute of $q_{2}$, is chosen as the value component of the schema. In addition, the attribute Product.name that is an ordering attribute of $q_{2}$ is chosen as a 


\begin{tabular}{|c|c|}
\hline Functions & Description \\
\hline accessPoints $(G)$ & For a query path graph $G$, return a list of nodes as the corresponding access points. \\
\hline queryNums $(n)$ & For a node $n$, return a list of queries (just numbers) whose access point is $n$. \\
\hline scalarAttributes $(n)$ & For a node $n$, return a list of scalar attributes of the corresponding entity type. \\
\hline keyAttributes $(n)$ & For a node $n$, return a list of key attributes of the corresponding entity type. \\
\hline outNeigbors $(n)$ & For a node $n$, return a list of nodes as the corresponding out-neighbors. \\
\hline queryNums $(e)$ & For a directed edge $e$, return the corresponding label as a list of query numbers. \\
\hline equalityAttributes $(q N o)$ & For a query whose number is $q N o$, return a list of attributes for equality search. \\
\hline inequalityAttributes $(q N o)$ & For a query whose number is $q N o$, return a list of attributes for inequality search. \\
\hline projectionAttributes $(q N o)$ & For a query whose number is $q N o$, return a list of attributes to be printed. \\
\hline orderingAttributes $(q N o)$ & For a query whose number is $q N o$, return a list of attributes for ordering. \\
\hline
\end{tabular}

Table 3.3. A list of functions required to access a QPG

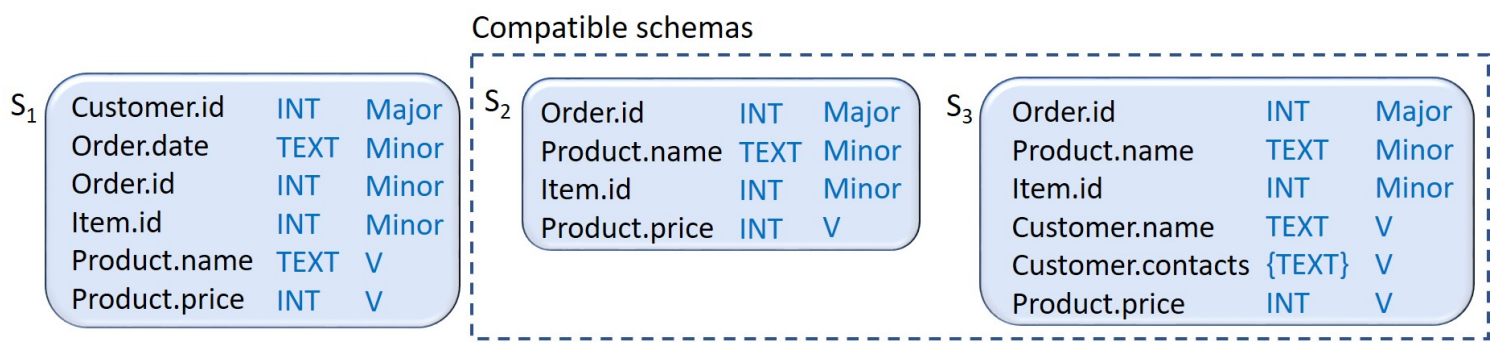

Fig. 3.8. The resulting KV schemas of mapping QPG in the running example

MINOR component. Regarding a similar mapping of QPG in the running example, Figure 3.8 depicts all generated KV schemas.

\section{Merging compatible KV schemas}

As shown in Figure 3.8, since the two KV schemas $S_{2}$ and $S_{3}$ share the same major key, their instances are sharded based on the same criteria (i.e., Order.id). In addition, as the schemas share the same minor key, the key-value pairs of each partition are ordered based on the same criteria (i.e., (Product.name, Item.id)). In this respect, 


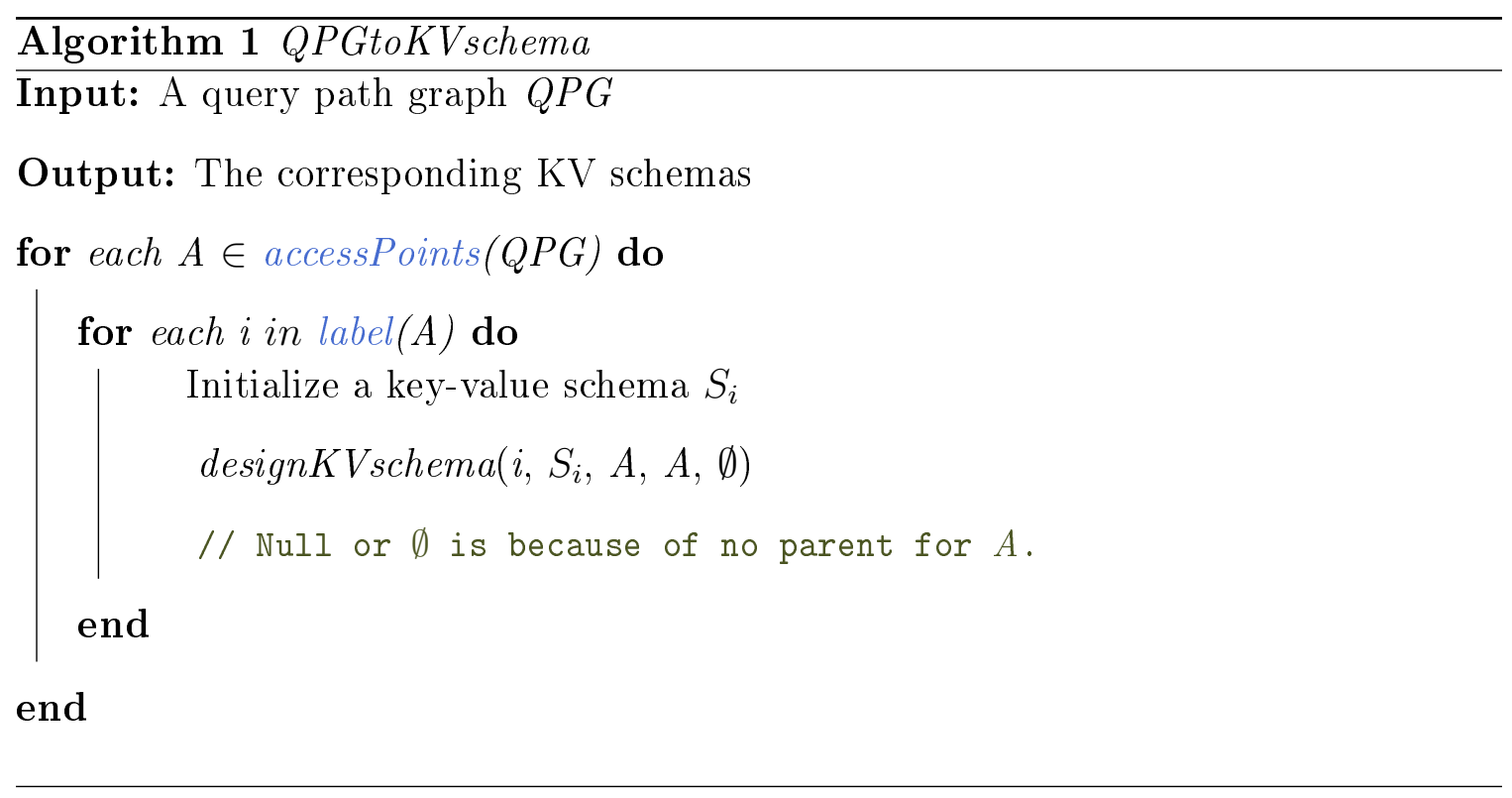

although the two schemas differ in their V components, merging the two schemas by combining these components can satisfy both the queries $q_{2}$ and $q_{3}$. This decreases the redundancy of Order (as an HW entity type) and improves the response time of write operations over it. However, it may impact the response time of $q_{2}$ as it needs to read and process more data than before (i.e., for a given Order, in addition to reading the data of related Product, it is required to read the extra data of corresponding Customer). Accordingly, we define compatible KV schemas as follows.

Definition 3.6. Given a workload with a set of query patterns $Q=\left\{q_{1} \ldots q_{n}\right\}$, where $S_{i}$ is the $\mathrm{KV}$ schema equivalent to $q_{i} ; K_{i}$ represents the sequence of MAJOR and MINOR components of $S_{i}$; and $V_{i}$ represents the set of $\mathrm{V}$ components of $S_{i}$, two schemas $S_{i}$ and $S_{j}$ are compatible iff $K_{i}$ is the same as $K_{j}$; hence, $S_{i}$ and $S_{j}$ can be merged into $S_{r}$ where $K_{r}=K_{i}$, and $V_{r}=V_{i} \cup V_{j}$.

The necessary condition for merging a pair of compatible schemas is to have at least one HW or HU entity type in common. Otherwise, it just impacts the read queries corresponding to the schemas with no pay-off for write operations or storage overhead. 


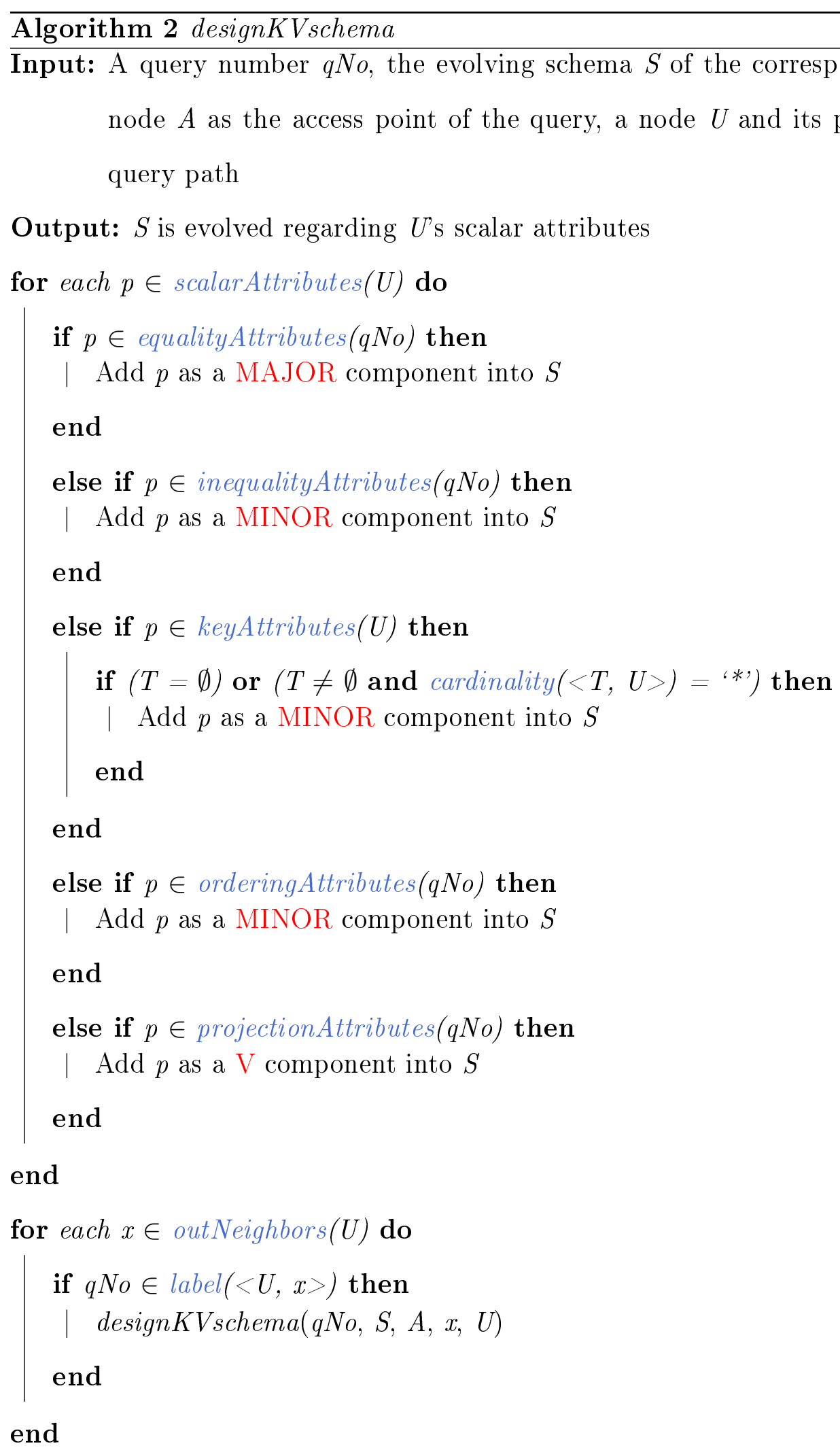




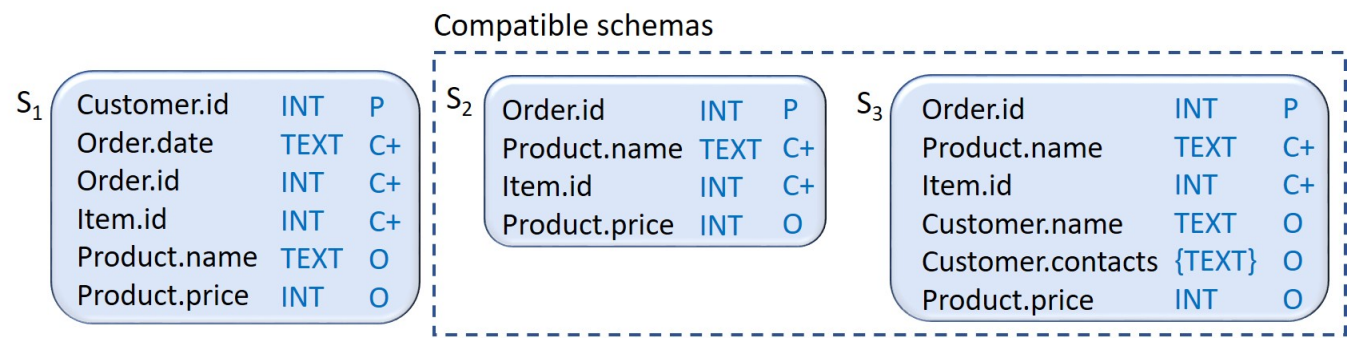

Fig. 3.9. The resulting CF schemas of mapping $\mathrm{QPG}$ in the running example

\subsubsection{Mapping QPG to CF schemas}

This section describes the process for automatically mapping a given QPG into a combination of CF schemas. This mapping process is described in detail in Algorithms 3 and 4. In more detail, Algorithm 4 follows the same pattern as Algorithm 2 by

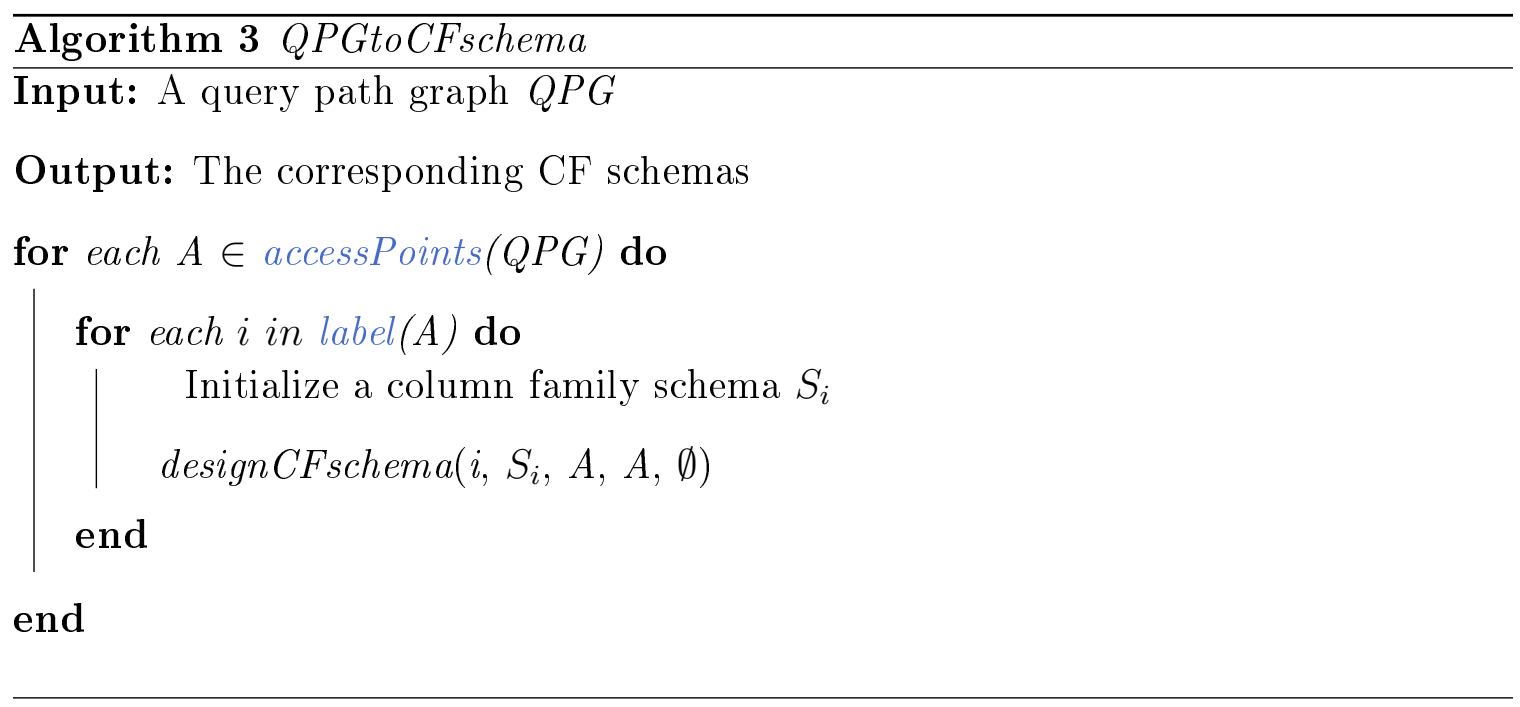

just replacing $M A J O R$ with $P$ and $M I N O R$ with $C$. This is due to the fact that in the schemas generated by these algorithms, MAJOR and $P$ components play the same role as sharding. Likewise, $M I N O R$ and $C$ components play the same role as ordering data in each shard. Figure 3.9 depicts all generated CF schemas, regarding the above algorithms. Furthermore, a similar reasoning as the one used for compatible KV schemas (see Section 3.3.5) is used for merging compatible CF schemas, as follows. 


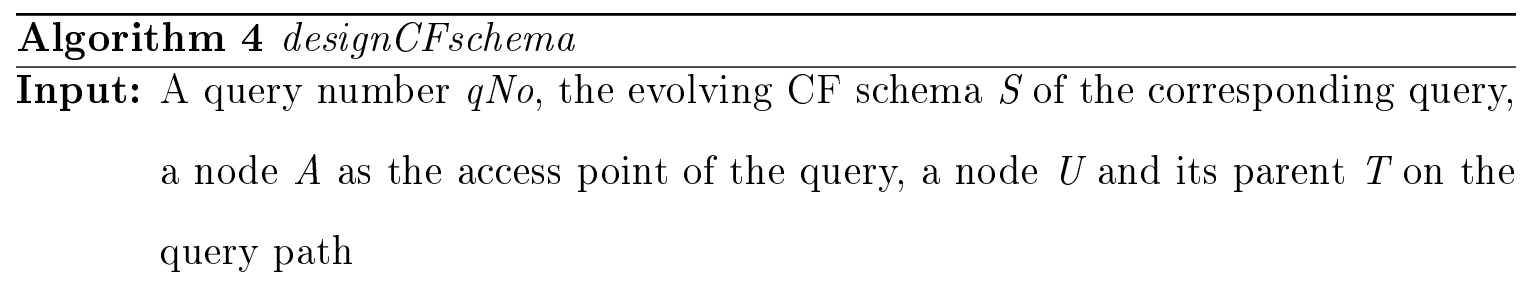

Output: $S$ is evolved regarding $U$ 's scalar attributes

for each $p \in$ scalarAttributes(U) do

if $p \in$ equalityAttributes (qNo) then

| Add $p$ as a P component into $S$

end

else if $p \in$ inequalityAtributes $(q N o)$ then

I Add $p$ as a C component into $S$

end

else if $p \in k e y$ Attributes $(U)$ then

if $(T=\emptyset)$ or $\left(T \neq \emptyset\right.$ and cardinality $\left.(<T, U>)={ }^{* *}\right)$ then

Add $p$ as a C component into $S$

end

end

else if $p \in$ projectionAttributes $(q N o)$ then

I Add $p$ as an O component into $S$

end

if $p \in$ orderingAttributes $(q N o)$ then

if $p$ is already added into $S$ then

| Reset $p$ as a $\mathrm{C}$ component with the corresponding $\langle+\rangle /<->$ order

end

else

Add $p$ as a $\mathrm{C}$ component into $S$ with the corresponding $\langle+>/<->$ order end

end

end

for each $x \in$ outNeighbors(U) do

if $q N_{o} \in \operatorname{label}(\langle U, x\rangle)$ then

I designCFschema $(q N o, S, A, x, U)$

end 
Definition 3.7. Given a workload with a set of query patterns $Q=\left\{q_{1} \ldots q_{n}\right\}$, where $S_{i}$ is the $\mathrm{CF}$ schema equivalent to $q_{i} ; K_{i}$ represents the sequence of $\mathrm{P}$ and $\mathrm{C}$ components of $S_{i}$, two schemas $S_{i}$ and $S_{j}$ are compatible iff $K_{i}$ is the same as $K_{j}$; hence, $S_{i}$ and $S_{j}$ can be merged into $S_{r}$ where $K_{r}$ is the same as $K_{i}$, and the set of ordinary components of $S_{r}$ consist of the union of the the corresponding sets in $S_{i}$ and $S_{j}$.

For example, in Figure 3.9, the two CF schemas $S_{2}$ and $S_{3}$ are compatible. Regarding the aforementioned necessary condition for merging compatible schemas, since both the schemas have Order (as an HW entity type) in common, they can be merged into a single one.

\subsubsection{Mapping QPG to document collection schemas}

This section describes the process for automatically mapping a given QPG into a combination of document collection schemas. This mapping process is described in detail in Algorithms 5 and 6, where we utilize data denormalization for the generation of document database schemas despite the work of [92].

As indicated in Section 3.1.4, document databases aim at improving the performance of read queries by storing nested entity hierarchies. As such, when a query asks for a hierarchy of nested entities, a single well-defined location can be accessed to retrieve the hierarchy. This avoids accessing different locations of the database and joining the retrieved information to produce the hierarchy. Hence, when designing a document database schema, we need to identify what hierarchies need to be stored in the generated databases schema. To do so, we consider each subgraph of QPG that is rooted by an access point, that consists of the corresponding query paths. This subgraph implies hierarchies of entities that need to be retrieved by the queries with the same entry point. Accordingly, we create a document collection for each access point. 


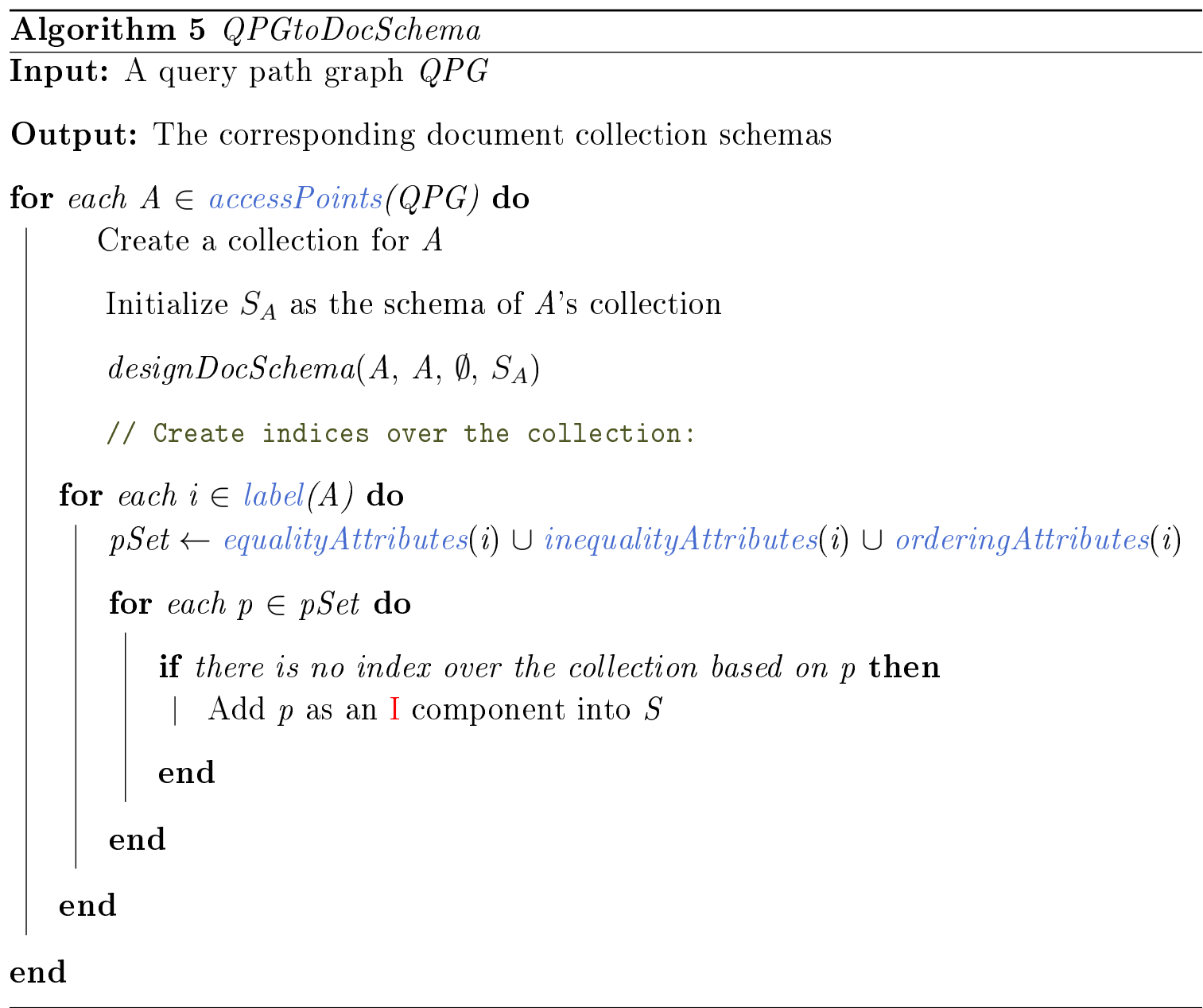




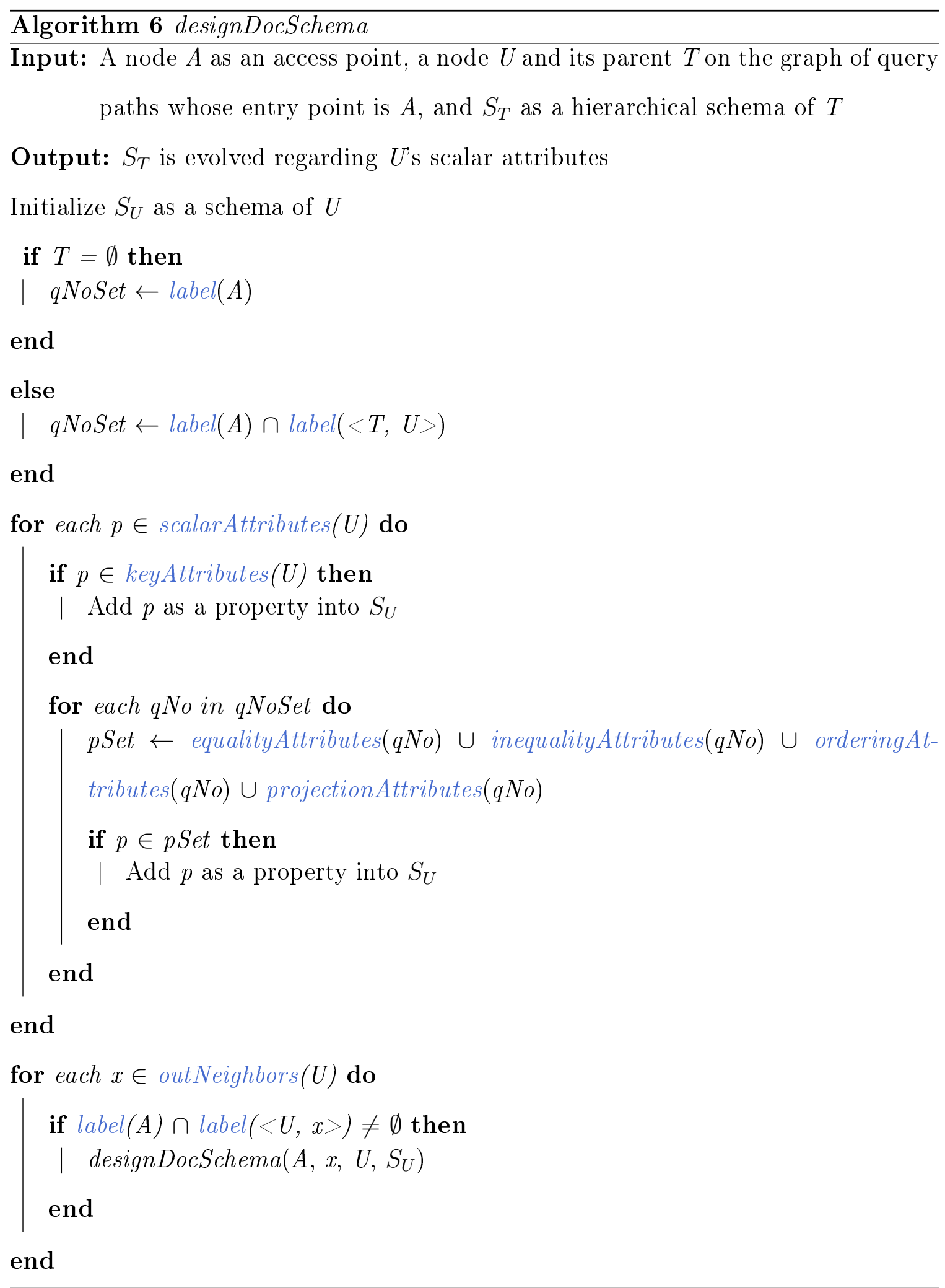




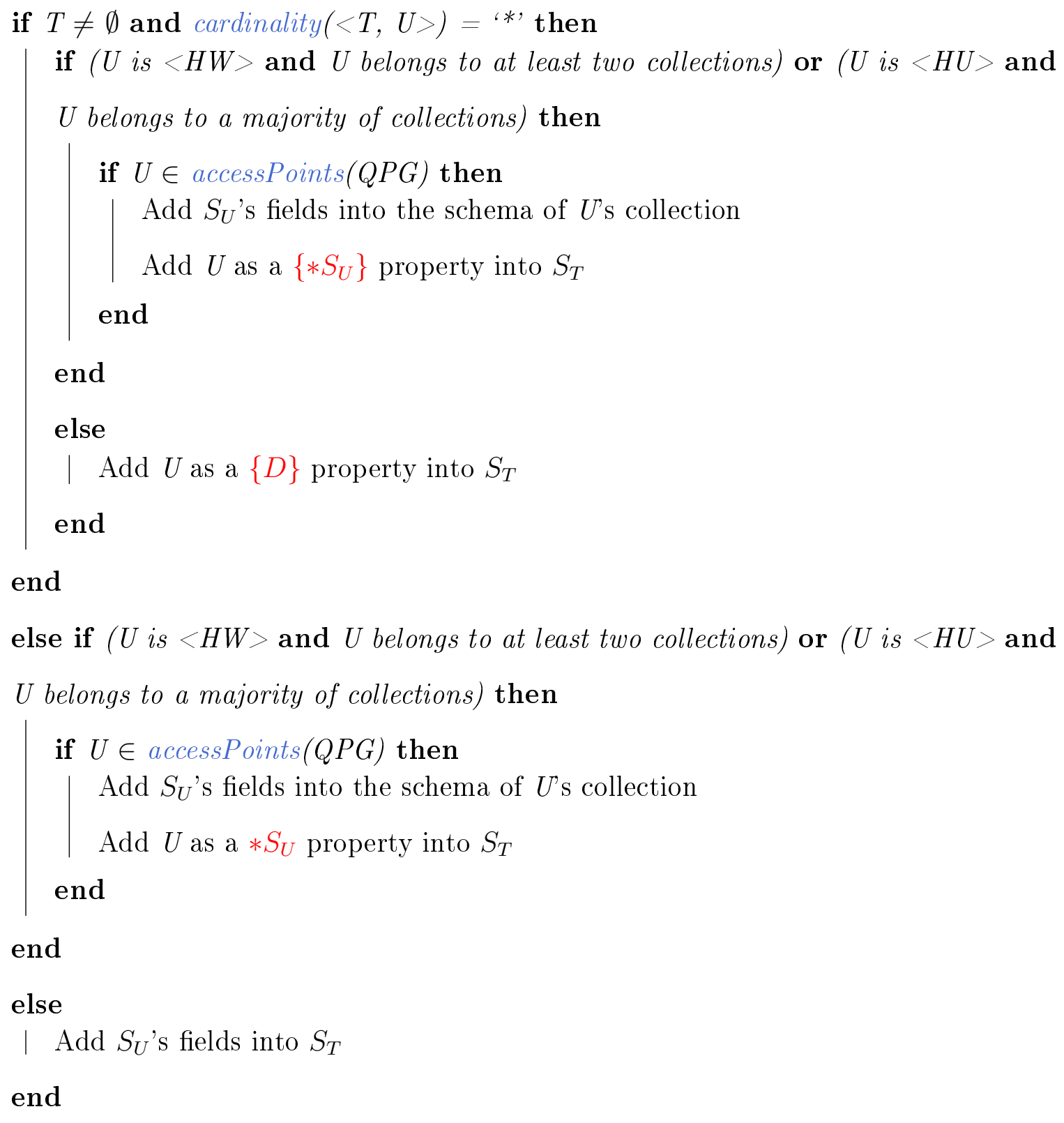




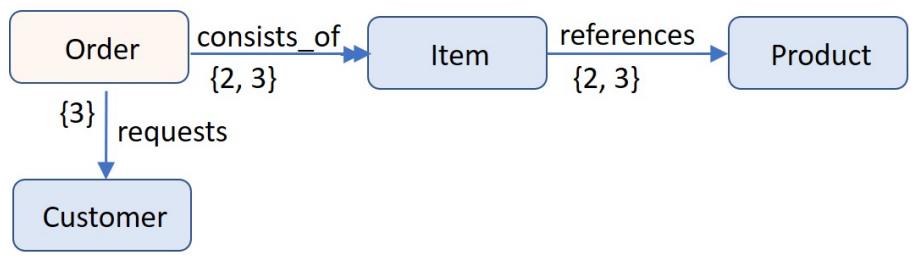

Fig. 3.10. The graph of query paths whose entry point is Order

For instance, Figure 3.10 depicts a subgraph of the QPG in our running example (see Figure 3.7(b)), rooted by Order. It consists of the paths of two queries q2 and q3. Now, let us dig into the mapping of this subgraph into a document collection. A document schema [98], named $S_{\text {Order }}$, is created for Order as an access point and is initialized with an empty set of fields. The node Order is firstly processed, whereby its key attribute Order.id is added to the fields of $S_{\text {Order }}$. The node Customer is then visited via the edge requests, whereby attributes Customer.name and Customer.contacts, that are required by $q_{3}$, are added to $S_{\text {Order }}$. Subsequently, the node Item is visited via the edge consists_of whose cardinality implies the association of many Items to an Order. Hence, an array of Items, called ItemList, is added to the fields of $S_{\text {Order }}$. Finally, an index on Product.name, belonging to the ordering keys of $q_{2}$ and $q_{3}$, is created over $S_{\text {Order }}$. Regarding a similar mapping for the other access point in the QPG (i.e., Customer), Figure 3.11(a) depicts the generated schemas for two document collections: $S_{\text {Customer }}$ and $S_{\text {Order }}$.

\section{Referencing vs. embedding}

The above mapping strategy embeds all required information to increase the performance of read operations. For example, as shown in Figure 3.11(a), the processing of $q_{1}, q_{2}$ or $q_{3}$ entails accessing a single collection, where $q_{1}$ accesses $S_{\text {Customer }}$ and either $q_{2}$ or $q_{3}$ accesses $S_{\text {Order }}$. However, this could result in a high level of denormalization that, in turn, might increase consistency maintenance and impact write operations. 
(a)

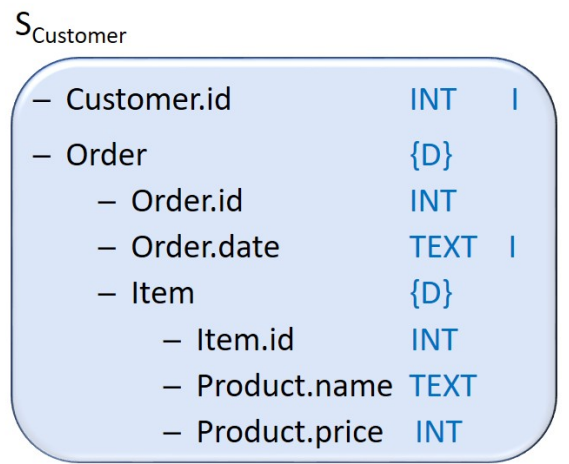

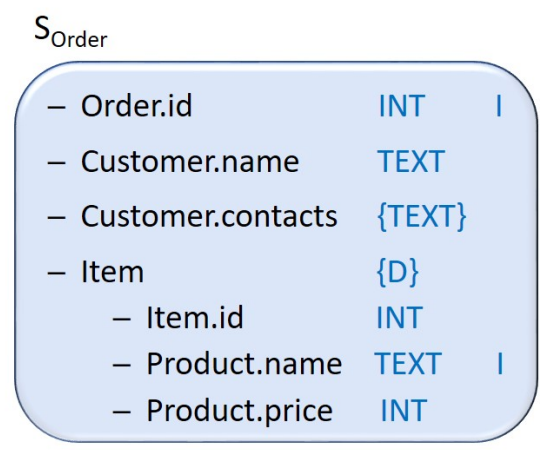

(b)
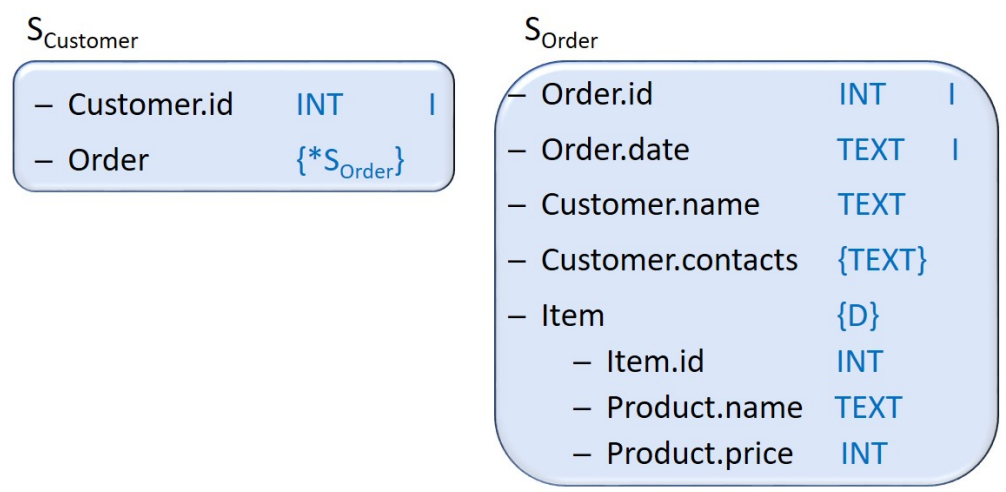

Fig. 3.11. (a) the resulting document collection schemas of mapping QPG in the running example; (b) the same mapping with respect to Order as an HW entity type

For instance, as shown in Figure 3.11(a), performing an update operation on Order, such as the insertion of the corresponding Items, necessitates accessing to the both collections.

Therefore, the mapping strategy might prevent the denormalization of HW and HU entities. The first prevention aims at increasing the performance of write operations as they are frequently performed on HW entities. The second prevention aims at reducing storage overhead as it can be increased by denormalizing HU entities. In this respect, assume an HW or HU entity type that is also an access point. Hence, the corresponding entities can be referenced rather than embedded, as our mapping strategy creates a referenceable collection for each access point. Also assume that either the HW or HU entity type appears in at least two collections (as even a replication 
degree of two might noticeably impact write operations on HW entities) or a majority of collections (as it might noticeably effect the size of database) respectively. As such, our mapping strategy exploits references to the corresponding entities instead of embedding them.

For instance, Figure 3.11(a) shows that Order, that is assumed to be an HW entity type, is appeared in two collections: $S_{\text {Order }}$ and $S_{\text {Customer }}$. Hence, we can utilize references to the instances of Order instead of embedding them. In this respect, Figure 3.11(b) depicts how our mapping strategy can utilize references to Orders instead of embedding them. Here, the update operation on Order requires accessing just one collection $S_{\text {Order }}$. However, this impacts the performance of $q_{1}$ as its processing entails accessing to the both collections.

\subsubsection{Computational complexity}

This section discusses the required computational resources of our automatic generation method. In the case of key-value or wide-column data models, the process starts by generating a $\mathrm{KV}$ or $\mathrm{CF}$ schema for each query via traversing the corresponding path and performing a set of basic calculations. In more detail, suppose there are $n$ entity types in the entity graph and $q$ query patterns in the workload of system. As the process traverses all query paths where each query path consists of at most $n$ entities, the corresponding time complexity is $\mathcal{O}(q \times n)$. Subsequently, the process compares pairs of generated schemas in order to combine compatible ones. The complexity of this stage, therefore, would be $\mathcal{O}\left(q^{2}\right)$. In normal scenarios, both the values $q$ and $n$ are presumed to be small, and there is a polynomial complexity of the above stages.

In the case of document data model, the process generates a document collection schema for each access or entry point via traversing the corresponding graph of query paths and performing a set of basic calculations. In more detail, as the graph of 
query paths for each access point is a DAG with at most $n$ nodes, by assuming $m$ is the number of edges in the graph, the time complexity of corresponding traversal is $\mathcal{O}(n+m)$. On the other hand, as the maximum number of access points is equal to $n$, the time complexity for the traversal of all graphs of query paths is $\mathcal{O}(n \times(n+m))$. As before, both values are supposed to be small, and there is a polynomial complexity of this stage as well.

As both the above processes are ended up with the generation of database schemas, we measured the times to generate $\mathrm{KV}, \mathrm{CF}$ and document database schemas in the aforementioned case studies. The generation times are about a few seconds, that is similar to the ones obtained by Mior et al [91] and de Lima and Mello [92].

\subsection{Summary}

In this chapter, we presented a novel workload-driven method for designing the schemas for different aggregate-oriented NoSQL databases from the same conceptual schema. This method is performed in two steps: 1) using some predefined rules to map QPG as a combination of the conceptual schema and the workload of the system into the constructs of key-value, wide-column and document NoSQL data models; and 2) using a set of rules to customize the generation of database schemas based on different context requirements. Here, we utilize extra information through HW and HU entity types, whereby the denormalization level of entities is controlled. This aims to make a trade-off between the cost of read operations with the cost of either write operations or storage. However, our method is not without limitations. There are still other criteria, such as dynamic workloads and the load-balance of the computing nodes (see Section 2.2.2), which should be taken into account by our system. In the next chapter, we introduce a partitioning method to overcome such limitations. 


\section{Chapter 4}

\section{Partitioning of Graph Databases}

This chapter focuses on our traversal-oriented strategy for the workload-driven partitioning of graph databases. As presented in section 2.2.3, streaming graph partitioning methods have recently gained attention due to their ability to scale to very large graphs with limited resources. However, many such methods do not consider workload and graph characteristics. This may degrade the performance of queries by increasing inter-node communication and computational load imbalance. Moreover, existing workload-aware methods cannot consistently provide good performance as they do not consider dynamic workloads that keep emerging in graph applications. We address these issues by proposing a novel workload-adaptive streaming partitioner named WASP, that aims to achieve low-latency and high-throughput online graph queries. As each workload typically contains frequent query patterns, WASP exploits the existing workload to capture active vertices and edges which are frequently visited and traversed respectively. This information is used to heuristically improve the quality of partitions either by avoiding the concentration of active vertices in a few partitions proportional to their visit frequencies or by reducing the probability of the cut of active edges proportional to their traversal frequencies. In order to assess the impact of WASP on a graph store and to show how easily the approach can be plugged on top of the system, we exploit it in a distributed graph-based RDF store. 


\subsection{Related work}

Although there are relevant works on workload-aware partitioning in RDBMS [99102], the main goal of such works is to avoid multi-table queries, and thus to avoid distributed transactions. In our partitioning method, the assumption is that there will be no multi-table queries at all. This assumption places those previous works in a different class of approaches. As a representative of such works, Curino et al. [99] propose a workload-driven approach for database partition and replication named Schism. Schism first generates a graph representation according to the workload and tuples in the database. The node in the graph represents the tuple, and the edge weight account for the frequency of co-accessing the two tuples. Schism partitions the graph using METIS. According to the partitioning result, Schism distributes and replicate the data. Our work is different from Schism in two-fold. First, the graph representation is different. We consider graph vertices instead of tuples. Second, Schism is interested in distribution and replication of SQL database. In this work, we focus on how to partition the vertices and edges of a graph database.

In recent years, several online partitioning strategies have been proposed for supporting low-latency query execution of large-scale dynamic graphs. They aim at increasing the performance of either offline graph analytics as in [103-111], or online graph queries as in $[22-27,29,30,112-114]$ whose workload-driven ones are more relevant to our work in this study. As we only review a subset of graph partitioning methods, the interested readers are referred to the recent surveys on graph partitioning [115-117].

Hermes [22] is a workload-driven partitioning method, where each vertex knows the number of its neighbors in each partition, the weight of each partition and the aggregate weight of partitions. Nodes are balanced based on the weight of their hosted vertices, where the weight of a vertex indicates the frequency of queries towards it. 
Vertex reassignment is triggered when the weights of vertices change. The gain of reassigning a vertex from its source to a target partition is how many more neighbors it has in the target than the source partition. Peng et al. $[27,118]$ propose a workloaddriven partitioning method that mines frequent query patterns from a representative workload. Then it puts matches of the same frequent pattern into the same fragment to improve the workload throughout.

WARP [25] is a workload-driven replication method, whereby RDF triples are initially partitioned using METIS, regarding their subjects. It then uses a representative workload to replicate frequently accessed triples across the cluster using the n-hop guarantee method [119]. Given a user query, WARP determines its center vertex and radius. If the query is within the n-hop guarantee, WARP sends the query to all servers, which evaluate the query in parallel. Otherwise, the query is decomposed into subqueries for which a distributed query evaluation plan is created. Subqueries are evaluated in parallel by all servers and the results are sent to the master which combines them. Partout [26] is also workload-driven by extracting frequent query patterns from a representative workload and using them to partition the data into fragments.

Loom [31] is a streaming partitioning strategy that assumes a given workload of graph patterns and their relative frequencies. During the workload, it discovers common patterns of edge traversals. It then compares the sub-graph pattern matching queries against these common patterns and attempts to reduce inter-partition traversals of frequently traversed sub-graphs by allocating each match to a single partition. Taper [24] takes any given initial partitioning as a starting point, and iteratively enhances it by estimating traversal probabilities for a given path queries workload. These are then used to swap chosen vertices across partitions, and reducing the probability of inter-partition traversals.

Table 4.1 summarizes the state-of-the-art workload-driven partitioning strategies 
for supporting online graph queries. Loom, Partout, WARP, and the Peng et al. method are based on knowing a priori workload. Also, Taper assumes a given frequency of patterns in the existing path query workload. By exploiting this prior knowledge, the parts of the dataset that are targeted together by future queries can be highlighted. However, not only it might be practically difficult to have such knowledge in advance, but such strategies do not (properly) adapt to changes. This results in degrading the quality of partitions by evolving the workload while there is no repartitioning. Hermes takes into account a uniform frequency of edge traversals despite the non-uniform edge weights of real-world graphs. On the other hand, WARP extensively exploits the replication of graph vertices for improving their locality of access. However, maintaining replicas means additional metadata management, which in turn increases the system overhead. Replicas also become useless by changing the workload, which in turn increases the storage overhead. On the contrary, WASP can take any given initial partitioning and assumes nothing about the workload upfront. Moreover, it avoids additional storage overheads by using no replication. WASP also exploits the hyper-cut model to alleviate the load imbalance of high-degree vertices and improve parallelism.

\subsection{WASP framework}

In this section, we describe the design of WASP in more detail.

\subsubsection{Data and query model}

In this paper, data are represented by the property graph model, as it has gained wide acceptance and is used in many graph database systems such as Neo4j and Titan. It is defined as follows. 
Table 4.1. Workload-driven partitioning methods supporting online graph queries

\begin{tabular}{lllll}
\hline Method & Cut model & Initial partitioning & Workload-adaptive & Topology-aware \\
\hline Hermes [22] & Vertex-centric & Simple hashing & $\bullet$ & $\boldsymbol{x}$ \\
\hline Peng et al. [27] & Vertex-centric & Existing workload & $\bullet$ & $\boldsymbol{x}$ \\
\hline Partout [26] & Vertex-centric & Existing workload & $\bullet$ & $\boldsymbol{x}$ \\
\hline WARP [25] & Vertex-centric & METIS & $\boldsymbol{x}$ & $\boldsymbol{x}$ \\
\hline Loom [31] & Vertex-centric & Existing workload & $\bullet$ & $\boldsymbol{x}$ \\
\hline Taper [24] & Vertex-centric & METIS & $\bullet$ & $\boldsymbol{\checkmark}$ \\
\hline WASP & Hybrid-cut & Simple hashing & $\checkmark$ & \\
\hline
\end{tabular}

Full support $(\boldsymbol{})$, Limited support (\%), No support $(\boldsymbol{X})$

Definition 4.1. A property graph is a tuple $G=\left(V_{G}, E_{G}, L_{G}, \lambda_{G}, \sigma_{G}\right)$, where $V_{G}$ is a finite set of vertices; $E_{G} \subseteq V_{G} \times V_{G}$ is a finite set of directed edges; $L_{G}$ is a finite set of labels; $\lambda_{G}: V_{G} \cup E_{G} \rightarrow L_{G}$ is a total function that maps a (vertex or edge) identifier to a label; and $\sigma_{G}:\left(V_{G} \cup E_{G}\right) \times \operatorname{Pro}_{G} \rightarrow V a l_{G}$ is a partial function that maps a (vertex or edge) identifier and a property (or attribute) to a value, assuming that $\operatorname{Pro}_{G}$ and $V a l_{G}$ are a final set of properties and a set of values, respectively.

Intuitively, $G$ is a directed, labeled and attributed multigraph, where each vertex represents an entity and has a label or type as well as a (possibly empty) set of properties associated with this entity, and each edge represents a binary relationship between entities and has a label and some properties as well.

Online graph queries can be classified into two major types, namely path queries and pattern matching queries [10]. Hence, we assume a workload $W=\left\{<Q_{1}\right.$, $\left.f_{1}>,<Q_{2}, f_{2}>, \ldots,<Q_{n}, f_{n}>\right\}$ as a set of either path queries or pattern matching queries $[120,121]$ and their frequencies, processed by an exploration-based query processor $[122,123]$. A pattern matching query $Q$ follows the same structure as the property graph, but instead of allowing its vertices $V_{Q}$, edges $E_{Q}$, labels $L_{Q}$ and 
property values $\mathrm{Val}_{Q}$ to contain only constants, it permits variables as well. A path query, which determines the existence of a path connecting two vertices of a property graph, can be considered as a subset of pattern matching queries.

\subsubsection{Workload characteristics}

As workloads are usually dynamic (via changing the frequency of associated queries), the quality of graph partitions may degrade over time. Hence, WASP encodes workload characteristics into vertex and edge weights, according to the following definitions.

Definition 4.2. Given a property graph $G$, for each directional edge $\langle u, v\rangle \in$ $E_{G}$, where $u$ and $v$ are in $V_{G}$ and hosted on nodes $N$ and $M$ respectively, there is a traversal weight $\omega(\langle u, v\rangle)$ denoting the amount of data passed by traversing the edge.

In more detail, during the processing of a query $Q$, a traversal from the source vertex $u$ to the target vertex $v$ sends both $Q$ and $u$ from $N$ to $M$ where $Q$ 's processing continues, followed by receiving back the exploration result required by $u$. This weight starts from a default minimum value of 0 indicating that its corresponding passive edge has not yet been traversed. By traversing an edge during the existing workload, the edge weight is gradually increased along with decreasing the probability of cutting the edge.

Definition 4.3. Given a property graph $G$, for each vertex $v \in V_{G}$, where $v$ is

hosted on node $N$, there is an activity weight $\omega(v)$ that is equal to the total weight of $v$ 's incident edges.

In more detail, as any traversal towards or from $v$ requires the exploration of its neighborhood, $\omega(v)$ denotes the computing load imposed on $N$ by accessing the corresponding local indices (see Section 4.2.4). Accordingly, by visiting an active 

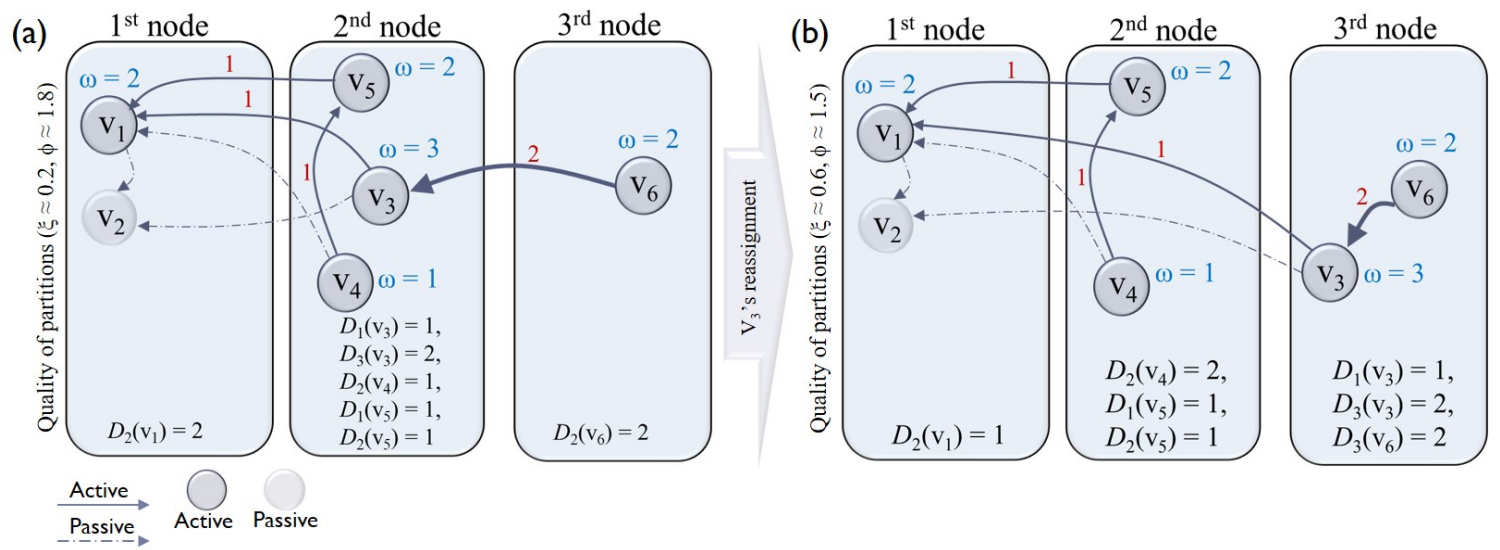

Fig. 4.1. (a) existing partitions before reassigning vertex $v_{3}$; (b) the improved quality of partitions after reassigning $v_{3}$ from the $2^{\text {nd }}$ to the $3^{\text {rd }}$ node

vertex during the existing workload, its activity weight becomes greater than 0 . As an illustration, Figure 4.1(a) shows the vertex/edge weights of a sample property graph. In this figure, the thicker the edges graphically indicate the ones with more frequent traversal.

\subsubsection{Vertex reassignment}

The Fennel streaming heuristic [78] is used for the online one-pass partitioning of large-scale graphs, whereby a newly added vertex is assigned (only once) to an existing partition with the highest number of its neighbours; while at the same time, a large partition should be penalized to prevent it from becoming too large with respect to the number of its hosted vertices. This heuristic is presented in Eq. 4.1, where $v$ refers to a vertex to be assigned, $N(v)$ refers to the set of $v$ 's neighbors, $V_{i}$ indicates the set of vertices hosted on the $i^{\text {th }}$ node, $n$ refers to the number of nodes, and $\alpha$ and $\gamma$ are parameters.

$$
\underset{1 \leq i \leq n}{\operatorname{argmax}}\left\{\left|N(v) \cap V_{i}\right|-\alpha \frac{\gamma}{2}\left(\left|V_{i}\right|\right)^{\gamma-1}\right\}
$$


Such a one-pass streaming heuristic is similar to the dynamic partitioning of graphs whose newly streamed vertices/edges are incrementally added to the existing partitions [78,124]. However, one-pass partitioning falls short in four areas to be workloadadaptive: (1) an assigned vertex is never reassigned, (2) the removal of vertices/edges is not considered, (3) a uniform frequency of edge traversals is considered and (4) partitions may not be balanced based on the aggregate activity weight of their hosted vertices. These drawbacks motivate us to use a workload-adaptive selective reassignment that continuously revisits active vertices, and reassigns them when appropriate. This necessitates maintaining some workload-based metadata in the main memory of computing nodes. This amount of information is not comparable to the huge amount of the given graph dataset. In more detail, according to the existing workload, assume $v \in S_{i}$, where $S_{i}$ is the set of active vertices hosted on the $i^{t h}$ node. The following metadata need to be maintained in the main memory of the $i^{\text {th }}$ node:

- $v$ 's degree of interest to be hosted on each node, where each degree is initialized from a default minimum value zero. In more detail, v's degree of interest towards the $j^{\text {th }}$ node $(j \in[1 . . n])$ is called $D_{j}(v)$, which indicates the total weight of $v$ 's incident edges to/from vertices hosted on the $j^{\text {th }}$ node. During a query traversal, $D_{j}(v)$ is incremented by sending a request from $v$ to a vertex on the $j^{\text {th }}$ node or vice versa. Accordingly, $\omega(v)$ can be simply calculated by summing up $v$ 's degrees of interest towards all nodes. Note that degrees with a default value of zero are not stored in the memory. They are not also shown in Figure 4.1.

- The activity weight of the $i^{t h}$ node, that is called $\omega\left(S_{i}\right)$. It indicates the aggregate activity weight of all active vertices hosted on the node.

- An edge $\log$ hosted on the $i^{\text {th }}$ node, that is called $\ell_{i}$. The log stores all active edges and corresponding weights, that are incident to the active vertices hosted on the $i^{t h}$ node. In more detail, during the existing workload, the most recently traversed 
edge incident to an active vertex hosted on the $i^{\text {th }}$ node is inserted on top of $\ell_{i}$. As a result, an edge $e$ that is not traversed any more (i.e., belonging to the previous workload) is gradually shifted to the bottom of the log and finally moved out of it. This turns $e$ into a passive edge and its weight is set to 0 , which in turn changes the degrees of locality of $e$ 's endpoint vertices. More precisely, assuming $e$ 's weight is $w$ and its endpoint vertices are $u$ hosted on the $j^{\text {th }}$ node, and $v$ hosted on the $i^{\text {th }}$ node, then $w$ is subtracted from both $D_{i}(u)$ and $D_{j}(v)$. $w$ is also subtracted from $\omega\left(S_{i}\right)$ and $\omega\left(S_{j}\right)$.

Each edge $\log$ has a configurable size $\Delta$, which is uniformly set for all nodes as their logs contain almost the same number of active edges. This is because of alleviating the load skew at query time through the edge-centric partitioning of high-degree vertices (see Section 4.2.3). When $\Delta$ is too small, each log stores a subset of active edges traversed in the existing workload. This means moving out some frequently traversed edges belonging to the workload and mistakenly making them passive. On the other hand, when $\Delta$ is too large, each log stores active edges that were traversed during the previous workloads, not the existing one. In both cases, the quality of partitioning may be impacted due to imprecise vertex reassignments. The choice and impact of $\Delta$ will be discussed in Section 5.2.6.

As aforementioned, our framework uses the simple hash partitioning scheme for the initial partitioning of the vertices across the nodes for two reasons. First, there is no complicated logic involved in assigning new vertices to partitions. Second, for a given vertex, we can simply look up its original hosting node. Hence, the initial node hosting a newly arrived vertex can be simply found through the hashing of the vertex $I D$. However, reassigning the vertex necessitates using a lookup table to find its new hosting node. This table can be implemented in a distributed manner through a set of lookup variables. More precisely, each reassigned vertex $v$ has a lookup variable stored as metadata in the $v$ 's initial node. Note that, as long as $v$ is hosted on its 
initial node, there is no need for this lookup variable.

Our selective reassignment heuristic is presented in Equation 4.2. By comparing to Equation 4.1, we set $\gamma=2$ and $\alpha=\frac{1}{\left|S_{i}\right| \times n}$.

$$
\begin{gathered}
\underset{1 \leq i \leq n}{\operatorname{argmax}}\left\{D_{i}(v)-\frac{\omega\left(S_{i}\right)}{\left|S_{i}\right| \times n}\right\} \\
\omega\left(S_{i}\right)=\sum_{1 \leq j \leq\left|S_{i}\right|} \omega\left(v_{j}\right), \text { where } v_{j} \in S_{i} \\
\omega\left(v_{j}\right)=\sum_{1 \leq i \leq n} D_{i}\left(v_{j}\right)
\end{gathered}
$$

This reassignment takes a vertex $v$ as input, computes a score for every node in the cluster, and a node with the highest score is determined as the potential target node to host $v$. To ensure a balanced partitioning with respect to the activity weight of nodes, there is a penalty function $\frac{\omega\left(S_{i}\right)}{\left|S_{i}\right| \times n}$, where by increasing the activity weight of each node, its score decreases.

In order to prevent the source node (assume it is the $s^{\text {th }}$ node) to be underloaded, the condition: $\left\{\omega\left(S_{s}\right)-\omega(v)\right\} \geq(2-\Phi) \times \Omega$ is checked before performing the selective reassignment. Here, $\Omega$ indicates the average aggregate activity weight of all nodes. In addition, parameter $\Phi \in[1,2]$, which is called maximum load imbalance, indicates how imbalanced a partition can be. For example, $\Phi=1$ indicates that all nodes are required to have the same aggregate of activity weights. If the source node is underloaded, the vertex $v$ is not considered to be moved. On the other hand, the condition: $\left\{\omega\left(S_{t}\right)+\omega(v)\right\} \leq \Phi \times \Omega$ is checked on the potential target node (assume it is the $t^{\text {th }}$ node) in order to prevent overloading it. If the node becomes overloaded, the condition is checked on the next highest score node; otherwise, the $t^{t h}$ node is selected as the target node.

Assume the quality of partitions is determined by a pair of quality factors: (1) the probability of intra-partition traversals $(\xi)$, and (2) the load imbalance factor $(\phi)$ indicating how imbalanced existing partitions are. These factors are defined as 
follows:

$$
\begin{gathered}
\xi=\frac{\text { the aggregate weight of edges that are not cut }}{\text { the weight of all edges }} \\
\phi=\frac{\max _{1 \leq i \leq n} \omega\left(S_{i}\right)}{\Omega}
\end{gathered}
$$

The selective reassignment improves $\xi$ as the higher the weight of an edge, the lower the probability to cut it. In other words, by increasing the weight of an edge, the interest of its endpoints to be collocated on the same node is increased. Higher the value of $\xi$ means less the IPT ratio which is equal to $1-\xi$.

Figure 4.1 illustrates the selective reassignment. Suppose there are three nodes, $\Phi=1.6$, and vertex $v_{3}$ is selected for reassignment. As Figure 4.1(a) shows, $\omega\left(S_{1}\right)=2$, $\omega\left(S_{2}\right)=6$, and $\omega\left(S_{3}\right)=2$, and $\Omega \approx 3.33$. With respect to Equation 4.2 , we have the following calculations: Score $_{1} \approx 0.33$, Score $_{2} \approx-0.66$, and Score $_{3} \approx 1.33$. As Figure 4.1(b) shows, after moving $v_{3}$, the $2^{\text {nd }}$ node (as the source node) is not underloaded $\left(\omega\left(S_{2}\right) \geq 1.32\right)$, and no one of the potential target nodes is overloaded (each one has an aggregate activity weight less than or equal to 5.33). As a result, $v_{3}$ is moved to the third node having the highest score. The quality of partitions after moving $v_{3}$ is improved as $\xi$ has increased from 0.2 to 0.6 , and $\phi$ has decreased from 1.8 to 1.5 in Figures $4.1(\mathrm{a})$ and (b) respectively.

\section{Vertex reassignment data maintenance}

Reassigning a vertex $v$ incurs moving its topological data from the $i^{\text {th }}$ node as the source to the $j^{\text {th }}$ node as the target. Such data includes $v$ 's relationships to other vertices along with $v$ 's properties. It also incurs maintaining the associated metadata as the following: (1) all active edges (and their weights) that are incident to $v$ are removed from $\ell_{i}$ and inserted to $\ell_{j}$, (2) $v$ 's degrees of interest towards all nodes are moved to the target node, (3) for each active edge $e$ that is incident to $u$ and $v, u$ 's degrees of interest towards the source and target nodes are changed. More precisely, $\omega(e)$ is subtracted from $D_{i}(u)$ and then added to $D_{j}(u),(4)$ the aggregate activity 
weights of the source and target nodes are changed. More precisely, $w$ is subtracted from $\omega\left(S_{i}\right)$ and added to $\omega\left(S_{j}\right)$, and (5) $v$ 's lookup variable in its initial node is updated to refer to the target node.

During the reassignment, the source node's query processor does not answer any request on $v$, in order to prevent their access to inconsistent data. As such, a request queue is maintained in the query processor, whereby all requests on $v$ are queued. By completing the reassignment, the $v$ 's lookup variable refers to the target node. Therefore, requests on $v$ are released from the queue and redirected to the target node.

\section{Vertex reassignment timing}

The timing of reassigning vertices is critical to make a balance between the quality of partitions and the above overhead of data/metadata maintenance. Recall that by changing the activity weight of a vertex, its degree of interest toward different nodes may change. This, in turn, triggers checking the possibility of reassigning the vertex. On the other hand, by increasing the activity weight of a vertex, the influence of a new exploration in its degree of interest towards different nodes is negligible. Therefore, after a reassignment, we will check the possibility of another reassignment only after a similar amount of new explorations. In more detail, assuming a reassignment threshold $k$, vertex reassignments are triggered after $\left\{k, 2 \times k, 4 \times k, \ldots, 2^{i} \times k\right.$, ... $\}$ explorations. This significantly reduces the number of reassignments for a vertex. For example, if $k$ is equal to 10 , for a vertex whose activity weight is 10,240 , the maximum number of reassignments is only 10. Currently, we use a hardwired reassignment threshold. The choice and impact of the reassignment threshold will be discussed in Section 5.2.5. 


\section{High-degree vertices}

High-degree active vertices may significantly reduce the efficiency and throughput of online querying. More precisely, their large neighbourhood may incur a significant processing overhead on their hosting nodes, as well as a large amount of network traffic on their incident edges. As an illustration, Fig. 4.2(a) depicts a high-degree active vertex $u$, hosted on node $N 1$, whose neighbors are scattered across $N 1$ to $N 3$. Also, assume there is a graph traversal through the pattern matching query $Q$, and vertices $t, u$ and $v_{1}$ to $v_{100,000}$ in the input graph are instances of vertices $T, U$ and $V$ in the query. Accordingly, a traversal from the source vertex $u$ to the target vertices $v_{1}$ to $v_{100,000}$ needs gathering and processing a huge amount of results that are sent back to $N 1$. In addition, a traversal from the source vertex $t$, hosted on node $N$ 2, to the target vertex $u$ incurs sending back the huge amount of gathered results from $N 1$ to N2.

WASP alleviates these issues by specifying and splitting high-degree vertices. Accordingly, for each vertex $u$ when $u$ 's degree (of incoming and outgoing edges) exceeds a configurable splitting threshold, it is considered as a high-degree vertex which in turn results in splitting up $u$ 's edges, whereby vertex $u$ is collocated with its neighbors. In more detail, an outgoing edge $u \rightarrow v$ is collocated with its target vertex $v$, and an incoming edge $u \leftarrow v$ is collocated with its source vertex $v$. Accordingly, as $u$ 's neighbors are randomly distributed through hashing, its edges will be evenly distributed. This uniformly divides the query processing overhead (which was already on $u$ 's hosting node) between all nodes that host its splits. As Fig. 4.2(b) shows, vertex $u$ is split into three vertices $u^{\prime}, u^{\prime \prime}$ and $u^{\prime \prime \prime}$ hosted on $N 1, N 2$ and N3, respectively. As such, traversing from $t$ as the source vertex to $u$ 's splits as the target neighbors results in dividing the traffic load (which was already between two nodes $N 1$ and N2) between all nodes. The choice and impact of the splitting threshold will be discussed 
(a)

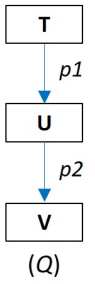

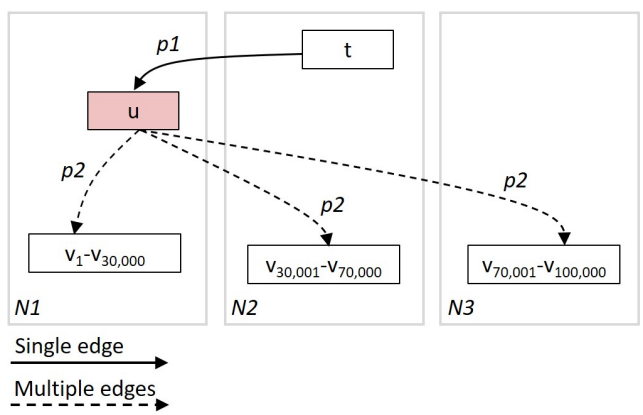

(b)

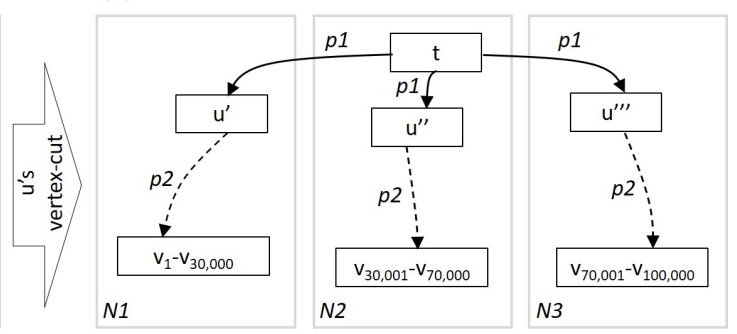

Fig. 4.2. (a) a high-degree active vertex $u$ regarding $Q$ as a query pattern belonging to the existing workload; (b) splitting up the edges of $u$ across the nodes

in Section 5.2.7.

\subsubsection{Verifying WASP on an RDF store}

The popularity of property graphs is due to their flexibility to express other structures. Accordingly, by not using attributes in property graphs, $\mathrm{RDF}^{1}$ or knowledge graphs are generated. Intuitively, an RDF graph consists of triples of the form $\langle$ subject, predicate, object $\rangle$ which can be interpreted as either two entities (subject and object) connected via a labeled relationship (predicate), or an entity (subject) associated via an attribute name (predicate) to its corresponding value (object). In a graph-based $\mathrm{RDF}$ store the dataset is stored as a graph, where RDF triples are modeled as vertices and edges [114]. An RDF graph is explored by walking the graph in specific orders according to the edges of a given SPARQL graph pattern matching query ${ }^{2}[122,123]$.

In order to assess the impact of WASP on a graph store and to show how easily the approach can be plugged on top of the store, it is exploited on a graph-based RDF store. WASP is deployed on a cluster whose nodes are connected in a peer-to-peer fashion similar to the one presented in Fig. 4.3(a). The key components of the system

\footnotetext{
1 http://www.w3.org/TR/rdf-primer/

2 http://www.w3.org/TR/rdf-sparql-query/
} 
(a)

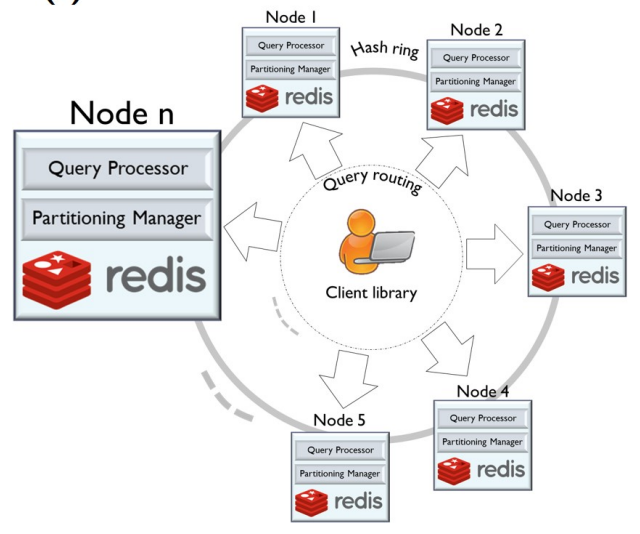

(b)

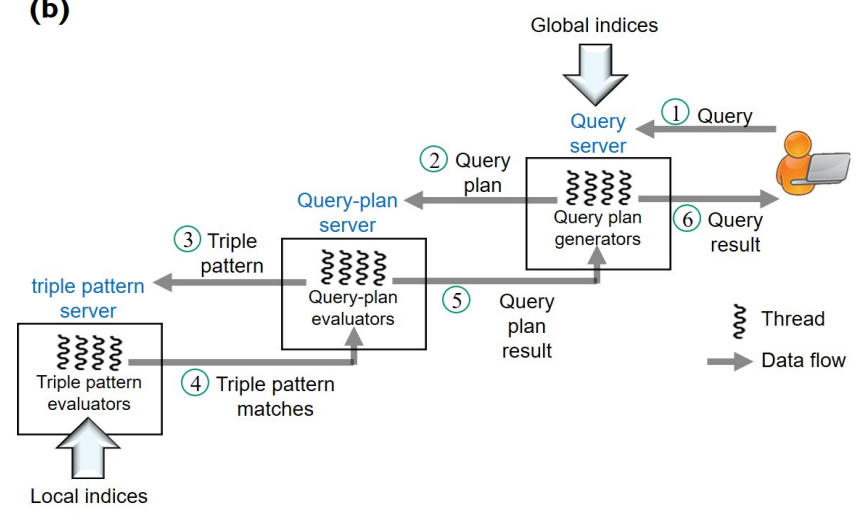

Fig. 4.3. (a) system architecture; (b) graph-based SPARQL query processing steps are as follows.

- A client library that contains a query routing module in order to balance the load among computing nodes. As such, a client can connect to any node and perform a query.

- A distributed memory storage that is made up of a batch of independent singlenode and in-memory Redis servers ${ }^{3}$ as the back-end stores. Redis provides a variety of data structures such as hashes, lists, sets, and ordered sets, as well as various operations for handling them. Accordingly, each node stores the associated data/metadata in the following local/global indices for an input graph $G$ (see Definition 4.1).

- Global index of vertices is stored as a DHT of KV pairs, where for each $v \in$ $V_{G}$, there is a $\mathrm{KV}$ pair consisting of the corresponding vertex identifier or vID as the key and the corresponding metadata as the value. Note that the name of $v$ is initially hashed to a $v I D$ which, in turn, is hashed to a computing node. In addition, the metadata of $v$ include its name and hosting node. Sending and storing $v I D s$ instead of long names may result in saving network bandwidth and

3 http://redis.io/ 
memory consumption. In addition, storing hosting nodes allows for changing the current hosting node of a vertex due to $G$ 's repartitioning.

- Global index of predicates is stored as a DHT of KV pairs, where for each $p$ $\in L_{G}$, there is a $\mathrm{KV}$ pair consisting of the corresponding predicate identifier or $p I D$ as the key and the corresponding metadata as the value. Note that the name of $p$ is initially hashed to a $p I D$. In addition, the metadata of $p$ include its name and a pair of lists <sbjNodeList, objNodeList $>$, where sbjNodeList (or objNodeList) includes node(s) hosting some subjects (or objects) incident to an edge labelled with $p$. This index is globally used to determine those nodes where a triple pattern with a bound predicate should be submitted for evaluation.

- Local index of vertices of each node is stored as a hash table of KV pairs, where each pair belongs to a vertex $v \in V_{G}$ that is hosted on the node. This pair consists of a combination of the corresponding $v I D$ and an OUT $(=1)$ or IN $(=0)$ direction as the composite key, and a list of all unique $p I D s$ of outgoing or incoming edges incident to $v$. This index is locally used for evaluating those triple patterns whose only subjects (or objects) are bound.

- Local index of predicates of each node is stored as a hash table of KV pairs, where each pair belongs to a predicate $p \in L_{G}$. This pair consists of a combination of the corresponding $p I D$ and an OUT or IN direction as the composite key, and a list of all vertices on the node that are the source or the target of an edge labeled with $p$, respectively. This index is locally used for evaluating those triple patterns whose only predicates are bound.

- Local index of vertices-predicates of each node is stored as a hash table of KV pairs, where each pair belongs to a vertex $v \in V_{G}$ that is hosted on the node. This pair consists of a combination of the corresponding $v I D$, a $p I D$ where $p \in L_{G}$ and an OUT or IN direction as the composite key, and the list of corresponding neighbor vertices as the value. This is used for evaluating those triple patterns 
whose only subjects (or objects) and predicates are bound.

- Local index of activity weights of each node is stored as a hash table of KV pairs, where each pair belongs to an active vertex $v \in V_{G}$ that is hosted on the node. This pair consists of a combination of the corresponding vertex identifier $v I D$ as the key, and the corresponding activity weight $(\omega(v))$ as the value.

- Local index of degrees of interest of each node is stored as a hash table of KV pairs, where each pair belongs to an active vertex $v \in V_{G}$ that is hosted on the node. This pair consists of a combination of the corresponding $v I D$ and a node identifier $n I D$ as the composite key, and v's degree of interest to be hosted on that node as the value.

- A graph-based SPARQL processor that consists of three running processes: 1) query plan generator that heuristically calculates a query plan for each received query, where a query plan is an ordered sequence of triple patterns. It then uses the aforementioned global indices to determine proper nodes where the plan is sent. Finally, after receiving the results of all sent plans, they are combined and sent back to the corresponding user; 2) query plan evaluator that receives a query plan and sequentially sends the corresponding triple patterns to proper nodes determined via the aforementioned global indices. Finally, after receiving the matches of all sent triple patterns, they are merged and sent back to the corresponding query plan generator; 3) triple pattern evaluator that uses the aforementioned local indices, determines the matches of a received triple pattern, and finally sends back the matches to the query plan evaluator. Fig. 4.3(b) depicts the query processing steps.

- WASP framework that is made up of several independent partitioning managers which are integrated with their peer query processors. Partitioners are in charge of watching the existing workload during the graph exploration along with making 
autonomous decisions for relocating graph vertices hosted on their corresponding nodes.

Note that since Redis servers are single threaded, by having each server manage partitions of both graph dataset and metadata, the concurrency between the processing of data and maintenance of metadata is decreased which in turn impacts the efficiency of query processing. As a result, we exploited two Redis servers on each node for the separate management of partitioned data and metadata.

\subsubsection{Memory and time complexities}

The amount of metadata used by WASP has a small size compared to the huge amount of a given graph dataset. In more detail, by storing the weights of at most $\Delta$ active edges on a node, the metadata of at most $2 \times \Delta$ active vertices are stored in the node as each edge represents two endpoint vertices. On the other hand, for each active vertex hosted on the node, there are at most $n$ degrees of interest towards all nodes. Therefore, there are at most $n \times 2 \times \Delta$ degrees of interest on each node. In addition, for each active vertex $u$ hosted on a node, there is an activity weight $\omega(u)$. Hence, there are at most $2 \times \Delta$ activity weights on each node. Finally, each node stores the total activity weight of its hosted active vertices requiring one long integer. As a result, the maximum amount of metadata used by WASP is $2 \Delta n^{2}+(3 \Delta+1) n$, where $n$ is the number of computing nodes and $\Delta$ is the size of the edge log. An implication of the above complexity is that the size of metadata scales with $\Delta$. However, according to our experiment in Section 5.2.6, $\Delta$ is far less than the number of edges of a given graph dataset. On the other hand, the performance of a partitioner is mainly affected by the amount of communication required by the partitioning algorithm. Accordingly, the time complexity of our partitioner relies on the number of times the selective reassignment and its examination are called. This requires a good balance between 
the edge-cut ratio and the number of vertex reassignments that is experimented in Section 5.2.5.

\subsection{Summary}

In this chapter, we presented WASP, a novel workload-adaptive and topology-aware streaming partitioner, in order to achieve low-latency and high-throughput online queries in distributed graph stores. As each workload typically contains popular or similar queries, WASP captures active vertices and edges that are frequently visited and traversed in the existing workload. Using this information, the quality of partitions is improved by avoiding the concentration of active vertices in a few partitions proportional to their visit frequencies, or by reducing the probability of the cut of active edges proportional to their traversal frequencies. However, our method is not without limitations. The performance of queries in static workloads can be increased by exploiting a workload-driven replication method, where the replication scheme of a vertex (i.e., how many replicas of the vertex are created, and to which partitions these replicas are allocated) dynamically changes based on the read/write frequencies of the vertex. 


\section{Chapter 5}

\section{Empirical Evaluations}

In previous chapters, we proposed two workload-driven methods for the key-oriented partitioning of aggregated oriented databases (Chapter 3), and the traversal-oriented partitioning of graph databases (Chapter 4). These methods are based on several new algorithms and techniques developed during this doctoral research, whereby their prototypes were developed as a full proof of concept. The aim of this chapter is to evaluate and subsequently validate the performances of the methods as part of our adopted DSRM methodology. In this regard, we conduct an interesting set of experiments to rigorously assess the efficiency and effectiveness of the methods.

\subsection{Key-Oriented partitioner evaluation}

We have implemented a prototype of the key-oriented partitioner to evaluate the mapping processes presented in Section 3.3. It is available under a free license in an external repository ${ }^{1}$. In addition, a detailed description of each subsequent case study along with the corresponding generated artifacts and measured response times are included. The target NoSQL stores are Oracle NoSQL version 15.5.19, Cassandra version 2.1.16 and MongoDB version 4.2.6 deployed on a Windows 10 machine

1 https://github.com/thaw-mon/QueryPathGraph.git 
equipped with an Intel Core i7-5500U CPU@2.4 GHz.

As aforementioned, the key-oriented partitioner mainly aims to automatically generate database schemas for various NoSQL stores from the same conceptual schema. As shown in Chapter 3, this goal has been achieved by the running example. In this section, we provide evidences for the general applicability of this method through its evaluation in other case studies. This is performed from the following three perspectives: (1) QPG expressiveness, (2) quality of generated database schemas compared with the related works, and (3) required computational resources.

\subsubsection{QPG expressiveness}

Since QPG mimics the notation of UML class diagrams, QPG's expressiveness should be as strong as that of UML which has been widely used for decades. Hence, it should be expressive enough as a generic logical model for aggregate-oriented NoSQL databases. We have modeled a set of external case studies with QPG to verify this hypothesis. Such case studies are commonly used in NoSQL research as testbeds. In particular, we have used three case studies: (1) a digital library used by Chebotko et al. [89], (2) an application in e-commerce domain used by de Lima and Mello [92], and (3) a cheat detection system for multiplayer games used by Mior et al. [91].

Table 5.1 provides a general overview of the case studies. As typical in NoSQL stores, despite the simplicity of case studies (e.g., small number of classes), it is intended to maintain a good performance of simple queries over voluminous data in a big data context. 
Table 5.1. Case studies for the aggregate-oriented NoSQL stores

\begin{tabular}{llll}
\hline Case study & Digital Library & Online Store & Cheat Detection \\
\hline \#Classes & 4 & 8 & 4 \\
\hline \#Associations & 6 & 7 & 3 \\
\hline \#Queries & 9 & 6 & 5 \\
\hline
\end{tabular}

\subsubsection{Quality comparison}

In this section, we compare the quality of database schemas generated using our method with the ones generated using other state-of-the-art methods. Before proceeding, it is worth noting that the main contribution of this paper is to create database schemas for various NoSQL data models using the same conceptual schema. However, we are interested in checking that the quality of generated database schemas has not been compromised by providing heterogeneity. In this respect, we selected those methods that are able to automatically generate database schemas for aggregateoriented NoSQL stores [89,91,92]. To be accurate, we used for the comparison the same case study (see Table 5.1) used in the paper that describes each selected method.

Note that our strategies for mapping QPG into document collections and CF constructs are strongly based on those available in the literature [89,91,92]. This leads to similar or even identical database schemas generated by our method and by the selected methods. The appeared differences are because of managing the trade-off between read query performance and storage overhead or consistency maintenance, regarding HW and HU entity types (see Sections 3.3.5 and 3.3.7).

\section{Digital library}

This case study is used to compare our method with the one proposed by Chebotko et al. [89]. Figure 5.1(a) and (b) show the conceptual schema of the digital library, as 
an entity graph (see Section 3.3.1), and the corresponding SQL-based query patterns (see Section 3.3.2) respectively. Using our method (see Definition 3.4), the entity graph and the workload of the digital library were initially mapped into a QPG. Figures 5.2(a) and (b) show the paths of queries in the workload of the digital library together with the corresponding QPG respectively. Tables 5.2(a), (b), and (c) depict the three specifications, regarding the QPG of the digital library. Finally, using our method for mapping QPG to CF schemas (see Section 3.3.6), the QPG was automatically mapped into a Cassandra implementation. More precisely, each query led to the generation of an individual CF schema; and the 9 CF schemas generated by our method were identical to the ones generated by Chebotko et al. [89] (see Figure 5.3). However, our method detected the two CF schemas generated for $Q 5$ and $Q 9$ as compatible schemas (see Definition 3.7). These schemas could then be merged (regarding Review as an HW entity type) into a single schema that, in turn, results in generating a single $\mathrm{CF}$ table to answer both queries.

In order to measure the effect of merging schemes on read and write operations, we compared the both designs by taking into account three operations: $Q 5, Q 9$ and a write operation that inserts an existing review. As Figure 5.4 shows, the performance of queries $Q 5$ and $Q 9$ in Chebotko's design is slightly more than our design. This is because both the queries in our design need to access a CF table with larger row size than individual tables accessed by the two queries in Chebotko's design. Hence, Cassandra has to process more data, that makes our design $0.4 \%$ slower in processing Q5 and Q9. However, while the insert operation accesses two tables in Chebotko, only one CF table is accessed in our design. This makes our design $48 \%$ faster in write operations. The performance of other read queries are not shown in Figure 5.4, as they access identical CF tables in both methods. 
(a)

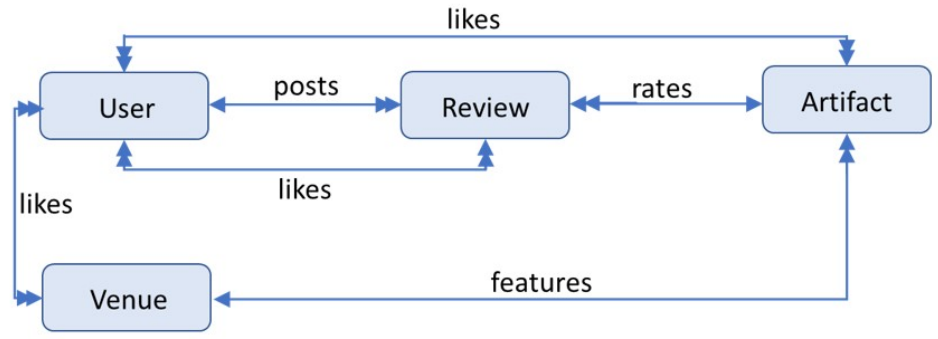

(b)

Q1 - SELECT Artifact.id, Artifact.title, Artifact.authors, Artifact.keywords FROM Venue.features.Artifact WHERE Venue. name $=$ ? AND Venue.year $>=$ ? ORDER BY Venue.year(DESC)

Q2 - SELECT Artifact.id, Artifact.title, Artifact.authors, Artifact.keywords, Venue.name FROM Artifact.features.Venue WHERE Artifact.author $=$ ? ORDER BY Venue.year(DESC)

Q3 - SELECT User.id, User.name, User.email, User.expertises FROM Artifact.likes.User WHERE Artifact.id = ?

Q4 - SELECT User.id, User.name, User.email, User.expertises FROM Artifact.likes.User WHERE Artifact.id = ? AND User.expertises = ?

Q5 - SELECT Review.rating FROM Artifact.rates. Review WHERE Artifact.id $=$ ?

Q6 - SELECT Venue.name, Venue.year, Venue.homepage, Venue.country, Venue.topics FROM User.likes.Venue WHERE User.id $=$ ?

Q7 - SELECT Artifact.title, Artifact.authors FROM User.likes.Artifact.features.Venue WHERE User.id $=$ ? AND Venue.year $>=$ ? ORDER BY Venue.year(DESC)

Q8 - SELECT Review.id, Review.rating, Review.body, Review.date, Review.title FROM User.posts. Review WHERE User.id $=$ ? AND Review.rating $>=$ ? ORDER BY Review.rating(DESC)

Q9 - SELECT Artifact.id, Artifact.title, Artifact.authors, Artifact.keywords FROM Artifact WHERE Artifact.id = ?

Fig. 5.1. (a) the digital library's entity graph; and (b) the corresponding workload 
(a)

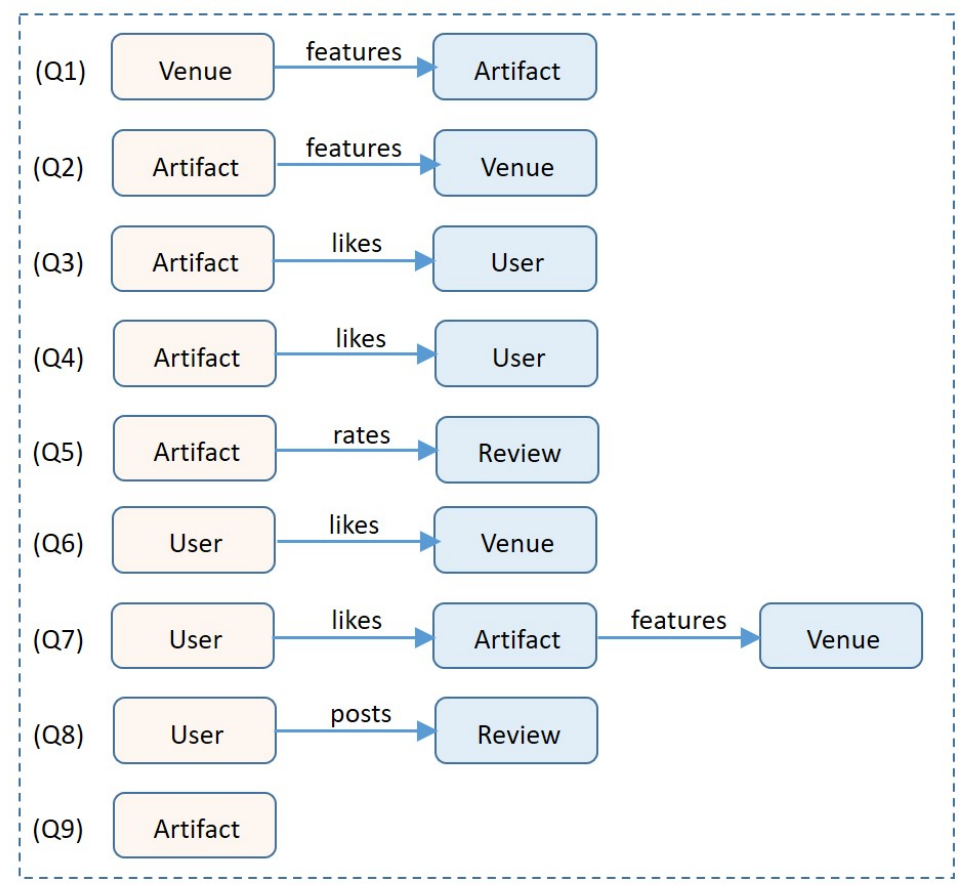

(b)

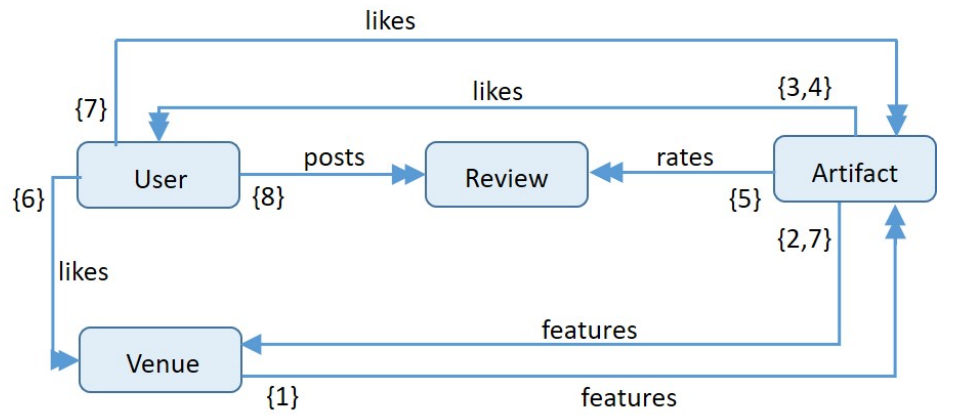

Fig. 5.2. (a) the paths of queries in the workload of the digital library; (b) the corresponding QPG

\section{Online store}

This case study is used to compare our method with the one proposed by de Lima and Mello [92]. Using our method regarding Order as an HW and Product as an HU entity type, the conceptual schema and the 6 read operations in the workload of the online store were initially mapped into a QPG that, in turn, was automatically mapped into a MongoDB implementation. Figure 5.5(a) and (b) show the conceptual 
(a)

\begin{tabular}{|c|c|c|c|c|c|}
\hline $\begin{array}{c}\text { QPG } \\
\text { node }\end{array}$ & $\begin{array}{c}\text { Scalar } \\
\text { properties }\end{array}$ & $\begin{array}{c}\text { Key } \\
\text { properties }\end{array}$ & $\begin{array}{c}\text { Access } \\
\text { point }\end{array}$ & $\begin{array}{c}\text { Query } \\
\text { numbers }\end{array}$ & Out neighbors \\
\hline Artifact & $\begin{array}{c}\text { Id/INT, title/STRING, } \\
\text { authors/STRING[], } \\
\text { keywords/STRING[] }\end{array}$ & id & Yes & $2,3,4,5,9$ & Venue, User \\
\hline User & $\begin{array}{c}\text { Id/INT, name/STRING, } \\
\text { email/STRING, } \\
\text { expertises/STRING[] }\end{array}$ & id & Yes & $6,7,8$ & $\begin{array}{c}\text { Venue, User, } \\
\text { Artifact }\end{array}$ \\
\hline $\begin{array}{c}\text { name/STRING, } \\
\text { year/INT, } \\
\text { homepage/STRING, } \\
\text { country/STRING, } \\
\text { topics/STRING[] } \\
\text { Id/INT, rating/INT, } \\
\text { body/STRING, } \\
\text { title/STRING, } \\
\text { date/STRING }\end{array}$ & id & name, year & Yes & 1 & Artifact \\
\hline Review & & & - & - \\
\hline
\end{tabular}

(b)

\begin{tabular}{|c|c|c|}
\hline QPG edge & $\begin{array}{c}\text { Query } \\
\text { numbers }\end{array}$ & Cardinality \\
\hline <User, Artifact>/likes & 7 & $*$ \\
\hline$<$ User, Venue>/likes & 6 & $*$ \\
\hline$<$ User, Review>/posts & 8 & $*$ \\
\hline$<$ Venue, Artifact>/features & 1 & $*$ \\
\hline <Artifact, User>/likes & 3,4 & $*$ \\
\hline$<$ Artifact, Venue>/features & 2,7 & 1 \\
\hline$<$ Artifact, Review>/rates & 5 & $*$ \\
\hline
\end{tabular}

(c)

\begin{tabular}{|c|c|c|c|c|}
\hline $\begin{array}{c}\text { Query } \\
\text { No }\end{array}$ & $\begin{array}{l}\text { Equality } \\
\text { properties }\end{array}$ & $\begin{array}{l}\text { Inequality } \\
\text { properties }\end{array}$ & $\begin{array}{l}\text { Ordering } \\
\text { properties }\end{array}$ & $\begin{array}{l}\text { Projection/aggregation } \\
\text { properties }\end{array}$ \\
\hline 1 & Venue.name & Venue.year & Venue.year[Desc] & $\begin{array}{l}\text { Artifact.id, Artifact.title, Artifact.authors, } \\
\text { Artifact.keywords }\end{array}$ \\
\hline 2 & Artifact.author & - & Venue.year[Desc] & $\begin{array}{l}\text { Artifact.id, Artifact.title, Artifact.authors, } \\
\text { Artifact.keywords, Venue.name }\end{array}$ \\
\hline 3 & Artifact.id & - & - & User.id, User.name, User.email, User.expertises \\
\hline 4 & $\begin{array}{l}\text { Artifact.id, } \\
\text { User.expertise }\end{array}$ & - & - & User.id, User.name, User.email, User.expertises \\
\hline 5 & Artifact.id & - & - & Artifact.sum_ratings, Artifact.num_ratings \\
\hline 6 & User.id & - & - & $\begin{array}{c}\text { Venue.name, Venue.year, Venue.homepage, } \\
\text { Venue.country, Venue.topics }\end{array}$ \\
\hline 7 & User.id & Venue.year & Venue.year[Desc] & Artifact.title, Artifact.authors \\
\hline 8 & User.id & Review.rating & Review.rating[Desc] & $\begin{array}{c}\text { Review.id, Review.rating, Review.body, Review.date, } \\
\text { Review.title }\end{array}$ \\
\hline 9 & Artifact.id & - & - & $\begin{array}{l}\text { Artifact.id, Artifact.title, Artifact.authors, } \\
\text { Artifact.keywords }\end{array}$ \\
\hline
\end{tabular}

Table 5.2. (a) specification of QPG nodes, (b) QPG edges and (c) QPG queries in the digital library 

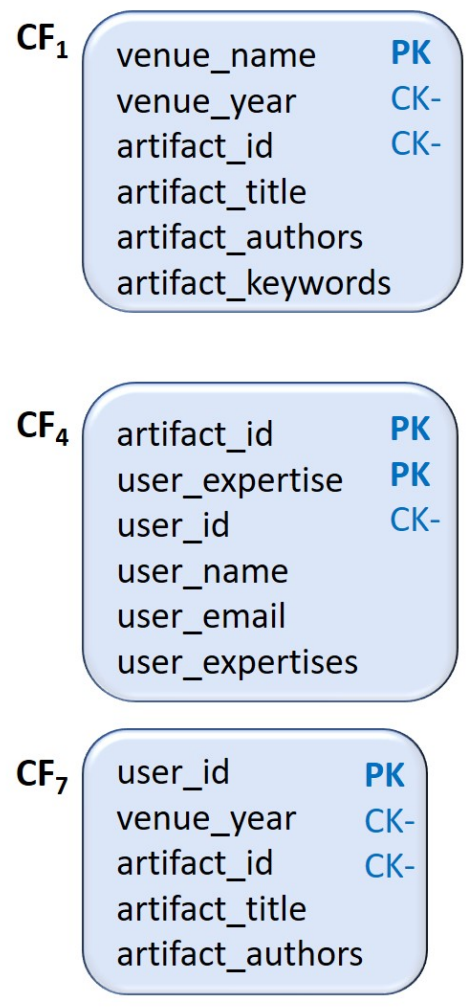

$\mathrm{CF}_{2}$
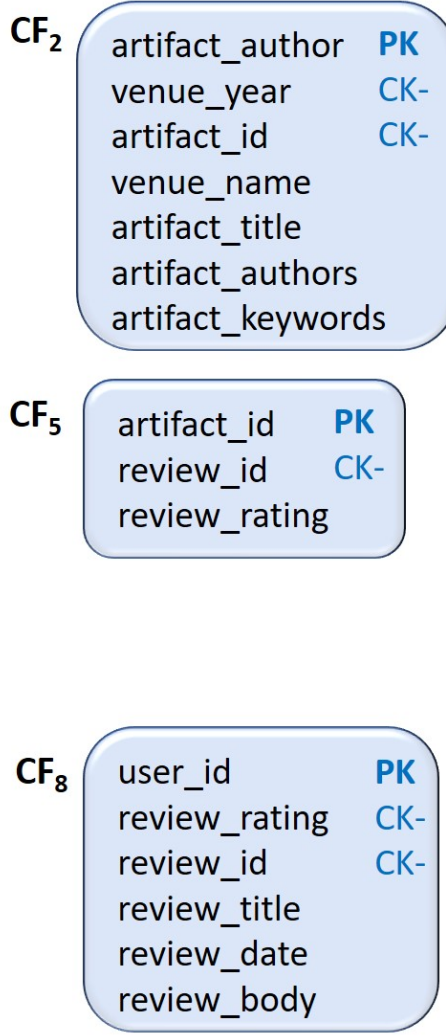
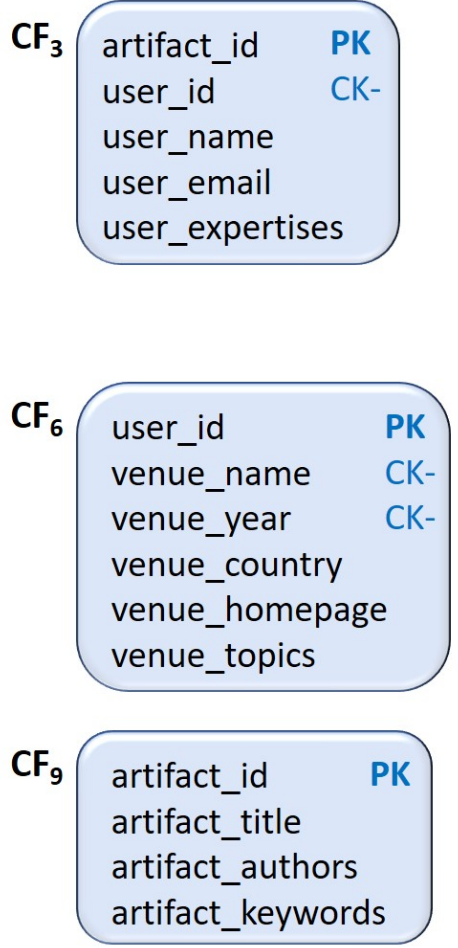

Fig. 5.3. The resulting CF schemas of mapping QPG in the digital library

schema of the online store and the corresponding SQL-based query patterns respectively. Figures 5.6(a) and (b) show the paths of queries in the workload of the online store together with the corresponding QPG respectively. Tables 5.3(a), (b), and (c) depict the three specifications, regarding the QPG of the online store. More precisely, each access point of the QPG led to the generation of a document collection (see Figure 5.7). However, the 4 collections generated by our method were different from the 5 collections generated by de Lima and Mello [92]. As described in Section 3.3.7, despite avoiding data redundancy in de Lima's method, our strategy can exploit a controlled denormalization of data regarding the HW and HU entity types.

Figure 5.8 illustrates the effect of this denormalization on the performance of read operations Operations $Q 1, Q 2, Q 4$ and $Q 5$ access the same collections in the both 


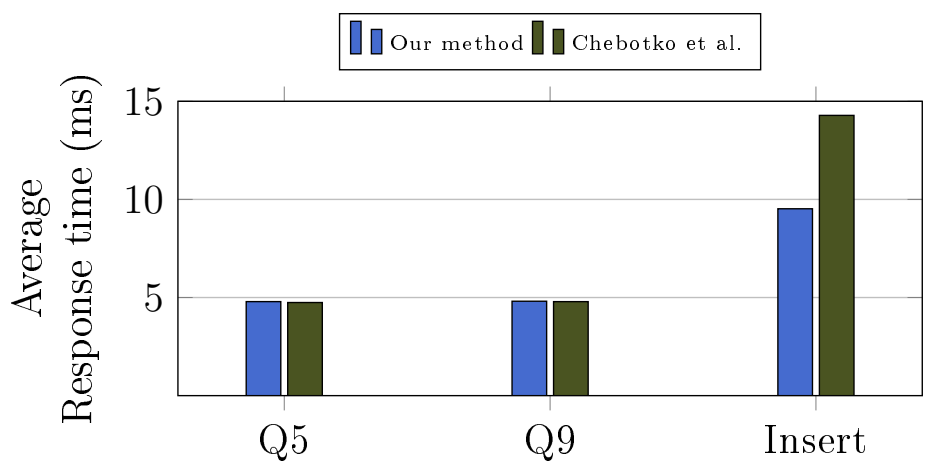

Fig. 5.4. Response times in our design vs. Chebotko et al.

(a)

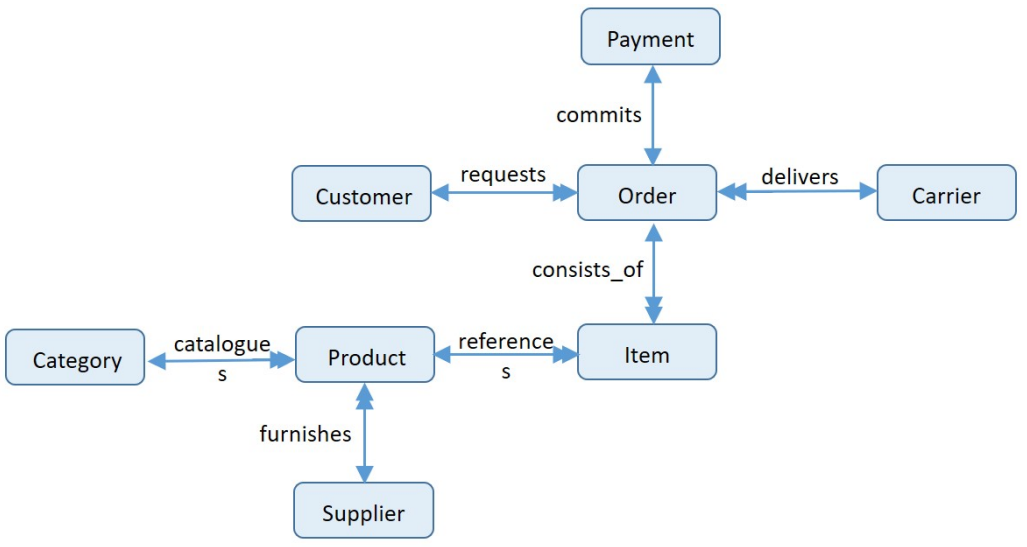

(b)

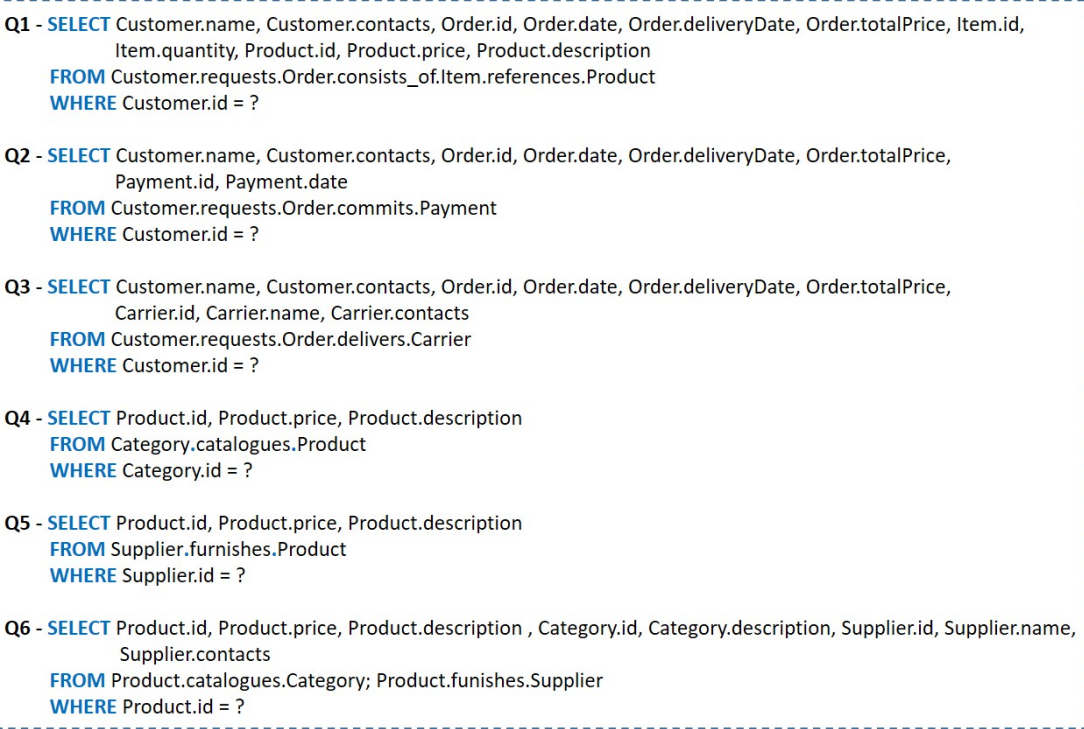

Q5 - SELECT Product.id, Product.price, Product.description FROM Supplier.furnishes.Product WHERE Supplier.id $=$ ?

Q6 - SELECT Product.id, Product.price, Product.description , Category.id, Category.description, Supplier.id, Supplier.name, Supplier.contacts FROM Product.catalogues.Category; Product.funishes.Supplier WHERE Product.id = ?

Fig. 5.5. (a) the online stores' entity graph; and (b) the corresponding workload 
(a)

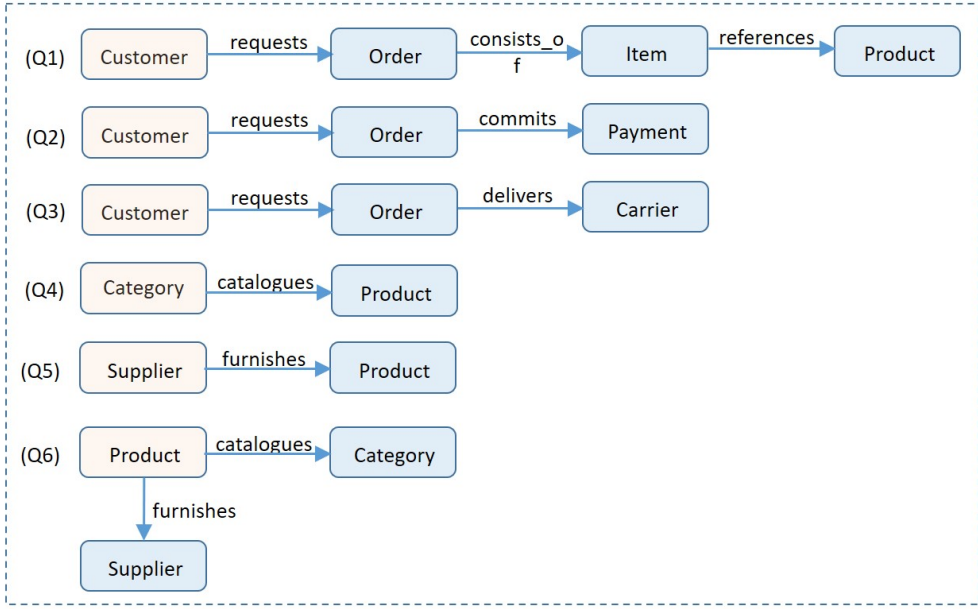

(b)

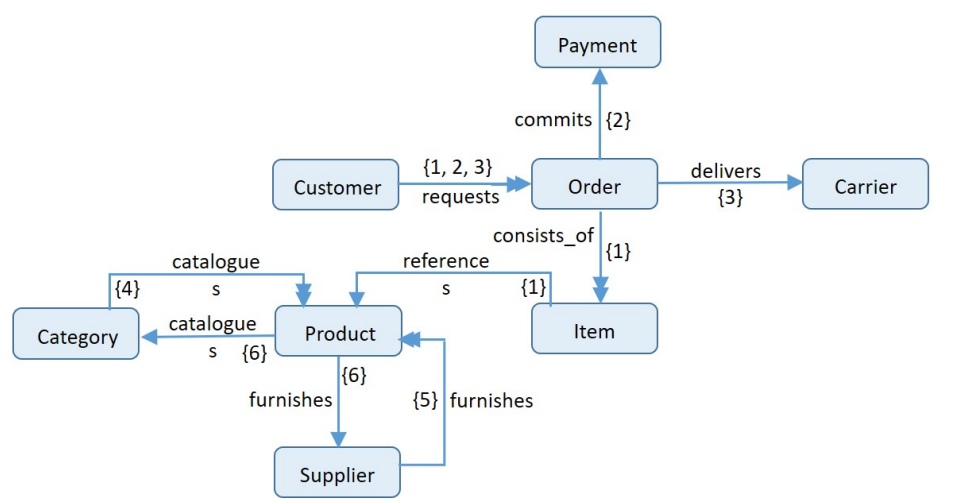

Fig. 5.6. (a) the paths of queries in the workload of the online store; (b) the corresponding QPG

designs. As the figure shows, while our method performs slightly worse than de Lima's in the performance of $Q 1$ and $Q 2$ (i.e., $2.7 \%$ and $4.8 \%$ respectively), for $Q 4$ and $Q 5$ it is basically equivalent to de Lima. This is due to accessing a document with larger size, because of the denormalization of data, that in tum requires processing more data by MongoDB. On the other hand, our design performs better than de Lima's in the performance of $Q 3$ and $Q 6$. In more detail, although both the queries access a single collection in our design, they have to access multiple collections in de Lima's design, that in tum require processing more data by MongoDB.

On the other hand, our design prevents the denormalization of Product as an 
(a)

\begin{tabular}{|c|c|c|c|c|c|}
\hline $\begin{array}{l}\text { QPG } \\
\text { node }\end{array}$ & $\begin{array}{c}\text { Scalar } \\
\text { properties }\end{array}$ & $\begin{array}{c}\text { Key } \\
\text { properties }\end{array}$ & $\begin{array}{c}\text { Access } \\
\text { point }\end{array}$ & Query numbers & $\begin{array}{c}\text { Out } \\
\text { neighbors }\end{array}$ \\
\hline Order & $\begin{array}{c}\text { Id/INT, totalPrice/INT, } \\
\text { date/STRING, } \\
\text { deliveryDate/STRING }\end{array}$ & id & No & - & $\begin{array}{c}\text { Payment, } \\
\text { Carrier, Item }\end{array}$ \\
\hline Customer & $\begin{array}{l}\text { Id/INT, name/STRING, } \\
\text { contacts/STRING[] }\end{array}$ & id & Yes & $1,2,3$ & Order \\
\hline Product & $\begin{array}{c}\text { Id/INT, price/INT, } \\
\text { description/STRING }\end{array}$ & id & Yes & 6 & $\begin{array}{l}\text { Supplier, } \\
\text { Category }\end{array}$ \\
\hline Supplier & $\begin{array}{l}\text { id, name/STRING, } \\
\text { contacts/STRING[] }\end{array}$ & id & Yes & 5 & Product \\
\hline Payment & Id/INT, date/STRING & id & No & - & - \\
\hline Carrier & $\begin{array}{l}\text { Id/INT, name/STRING, } \\
\text { contacts/STRING[] }\end{array}$ & id & No & - & - \\
\hline Category & $\begin{array}{c}\text { Id/INT, } \\
\text { description/STRING }\end{array}$ & id & Yes & 4 & Product \\
\hline Item & Id/INT, quantity/INT & id & No & - & - \\
\hline
\end{tabular}

(b)

\begin{tabular}{|c|c|c|}
\hline QPG edge & $\begin{array}{c}\text { Query } \\
\text { numbers }\end{array}$ & Cardinality \\
\hline <Customer, Order>/requests & $1,2,3$ & $*$ \\
\hline <Order, Payment>/commits & 2 & 1 \\
\hline <Order, Carrier>/delivers & 3 & 1 \\
\hline <Order, Item>/consists_of & 1 & $*$ \\
\hline <Supplier, Product>/furnishes & 4 & $*$ \\
\hline <item, Product>/references & 1 & 1 \\
\hline <Category, Product>/catalogues & 5 & $*$ \\
\hline <Product, Category>/catalogues & 6 & 1 \\
\hline <Product, Supplier>/furnishes & 6 & 1 \\
\hline
\end{tabular}

(c)

\begin{tabular}{|c|c|c|c|l|}
\hline $\begin{array}{c}\text { Query } \\
\text { No }\end{array}$ & $\begin{array}{c}\text { Equality } \\
\text { properties }\end{array}$ & $\begin{array}{c}\text { Inequality } \\
\text { properties }\end{array}$ & $\begin{array}{c}\text { Ordering } \\
\text { properties }\end{array}$ & \multicolumn{1}{c|}{$\begin{array}{c}\text { Projection/aggregation } \\
\text { properties }\end{array}$} \\
\hline 1 & Customer.id & - & - & $\begin{array}{l}\text { Customer.name, Customer.contacts, Order.id, Order.date, Order.deliveryDate, Order.totalPrice, } \\
\text { Item.id, Item.quantity, Product.id, Product.price, Product.description }\end{array}$ \\
\hline 2 & Customer.id & - & - & $\begin{array}{l}\text { Customer.name, Customer.contacts, Order.id, Order.date, Order.deliveryDate, Order.totalPrice, } \\
\text { Payment.id, Payment.date }\end{array}$ \\
\hline 3 & Customer.id & - & - & $\begin{array}{l}\text { Customer.name, Customer.contacts, Order.id, Order.date, Order.deliveryDate, Order.totalPrice, } \\
\text { Carrier.id, Carrier.name, Carrier.contacts }\end{array}$ \\
\hline 4 & Category.id & - & - & Category.description, Product.id, Product.price, Product.description \\
\hline 5 & Supplier.id & - & - & Supplier.name, Supplier.contacts, Product.id, Product.price, Product.description \\
\hline 6 & Product.id & - & - & $\begin{array}{l}\text { Product.id, Product.price, Product.description, Category.id, Category.description, Supplier.id, } \\
\text { Supplier.name, Supplier.contacts }\end{array}$ \\
\hline
\end{tabular}

Table 5.3. (a) specification of QPG nodes, (b) QPG edges and (c) QPG queries in the online store

HU entity type, whereby the overhead of storage is reduced. Comparing the cost of storage in the both designs shows that our design performs almost equal to that of de Lima. 


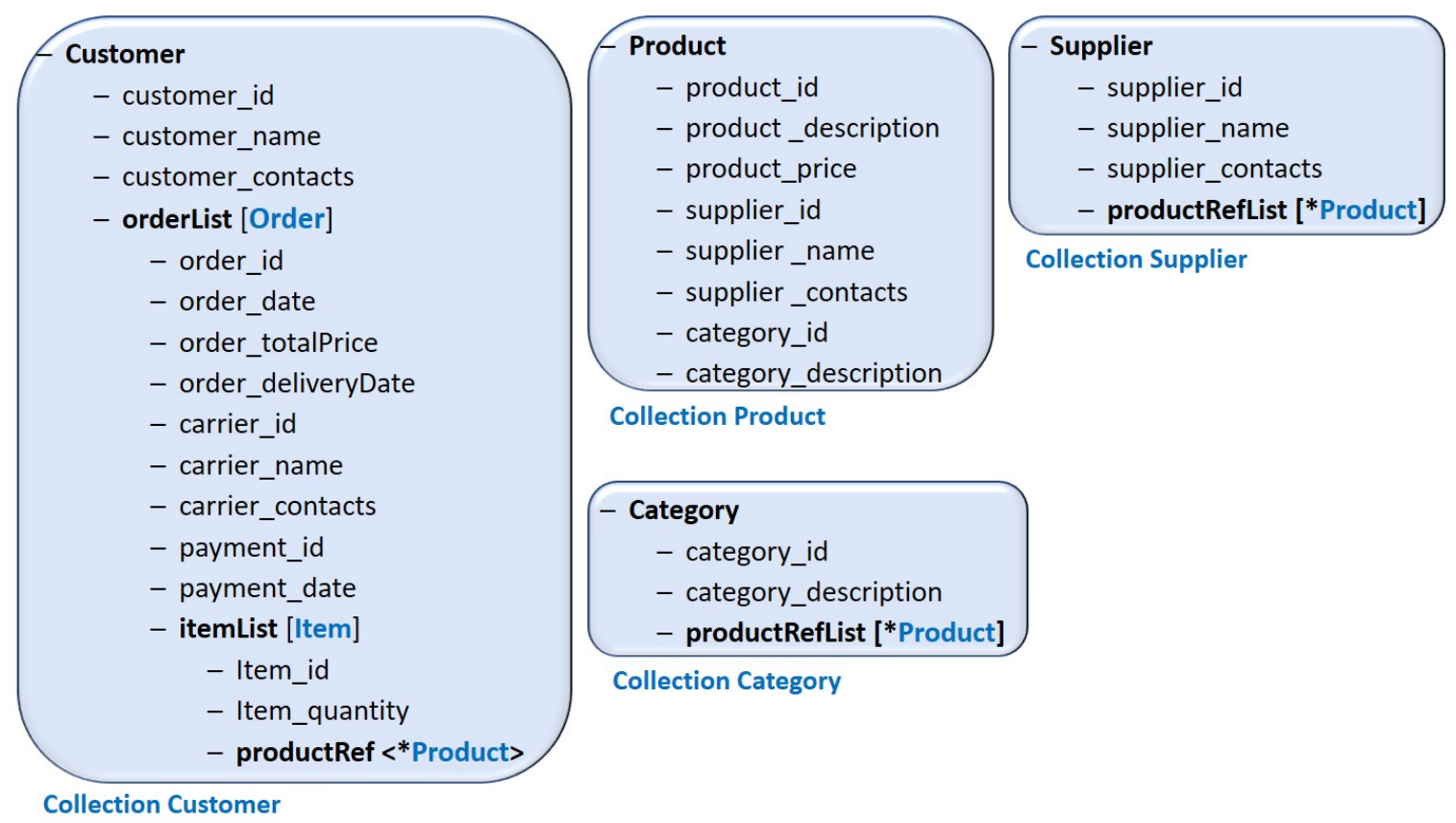

Fig. 5.7. The resulting document collection schemas of mapping QPG in the online store, with control on the denormalization degree of the Product entity type

\section{Easy anti cheat}

This case study ${ }^{2}$ is used to compare our method with the one proposed by Mior et al. [91]. Figure 5.9(a) and (b) show the conceptual schema of the easy anti cheat and the corresponding SQL-based query patterns respectively. Figures 5.10(a) and (b) show the paths of queries in the workload of the easy anti cheat use-case together with the corresponding QPG respectively. Tables 5.4(a), (b), and (c) depict the three specifications, regarding the QPG of the the easy anti cheat. Finally, using our method for mapping QPG to CF schemas (see Section 3.3.6), the QPG was automatically mapped into a Cassandra implementation. More precisely, each query led to the generation of an individual CF schema; and 5 CF schemas were generated by our method (see Figure 5.11).

2 https://www.easy.ac/en-us/ 


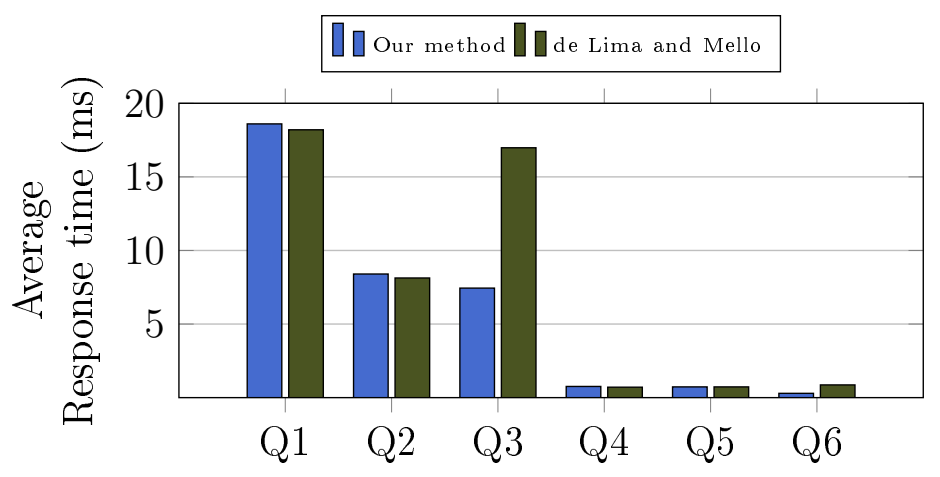

Fig. 5.8. Response times in our design vs. de Lima's

As already mentioned in Section 3.2, NoSE [91] takes into account the estimated frequencies of queries in existing workload and selects a Cassandra design with the best performance among all alternatives. Hence, a Cassandra design generated by our method is always included as one of the alternatives. In this experiment, we aim to quantify how our method could degrade performance compared to NoSE, assuming $80 \%$ of write operations and $20 \%$ of reads.

Using our method regarding Server as an HU entity type, each query led to the generation of an individual CF schema, where the schemas corresponding to $Q 3$ and Q4 are combined as compatible ones. As such, 3 out of the 4 generated schemas were identical to the ones generated by NoSE. However, the methods differ in the schemas generated for $Q 1$ and $Q 2$, where NoSE avoids denormalizing data by combining the two corresponding $\mathrm{CF}$ schemas. This combination improves writing performance as write operations need to access a single table; however, it necessitates some application-level computations to process read operations that, in turn, impacts their performance. In order to determine the effect of this denormalization, Figure 5.12 shows the performance of queries $Q 1$ and $Q 2$ as well as a write operation that updates an existing server in the both designs.

As expected, our method is better than NoSE in read query performance, especially in $Q 1$. However, in terms of update operation, our method is about $30 \%$ 
(a)

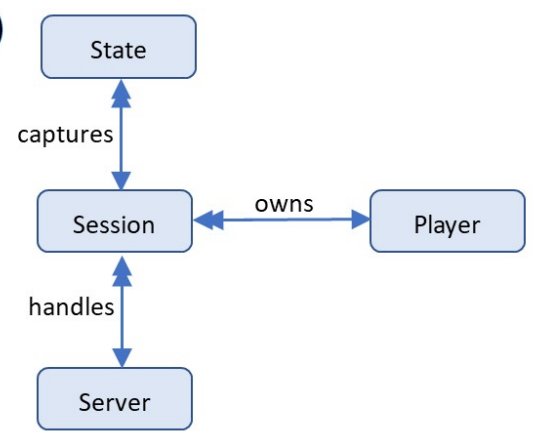

(b)

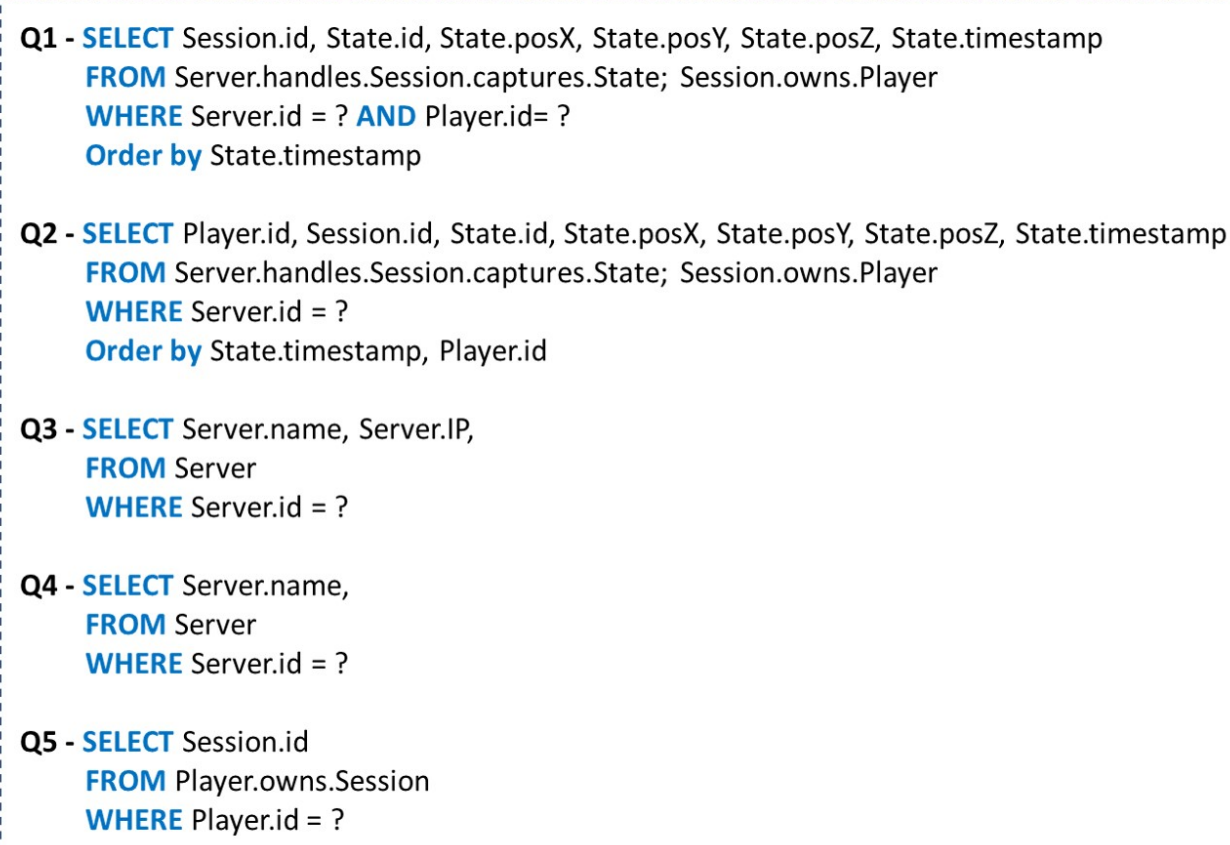

Fig. 5.9. (a) the the easy anti cheat's entity graph; and (b) the corresponding workload

slower, especially as most of operations are write operations. Note that since other queries access identical CF tables in both methods, there is no performance penalty on them. In summary, it can be claimed that while NoSE works best for write-intensive applications, our method fits read-intensive applications. 
(a)

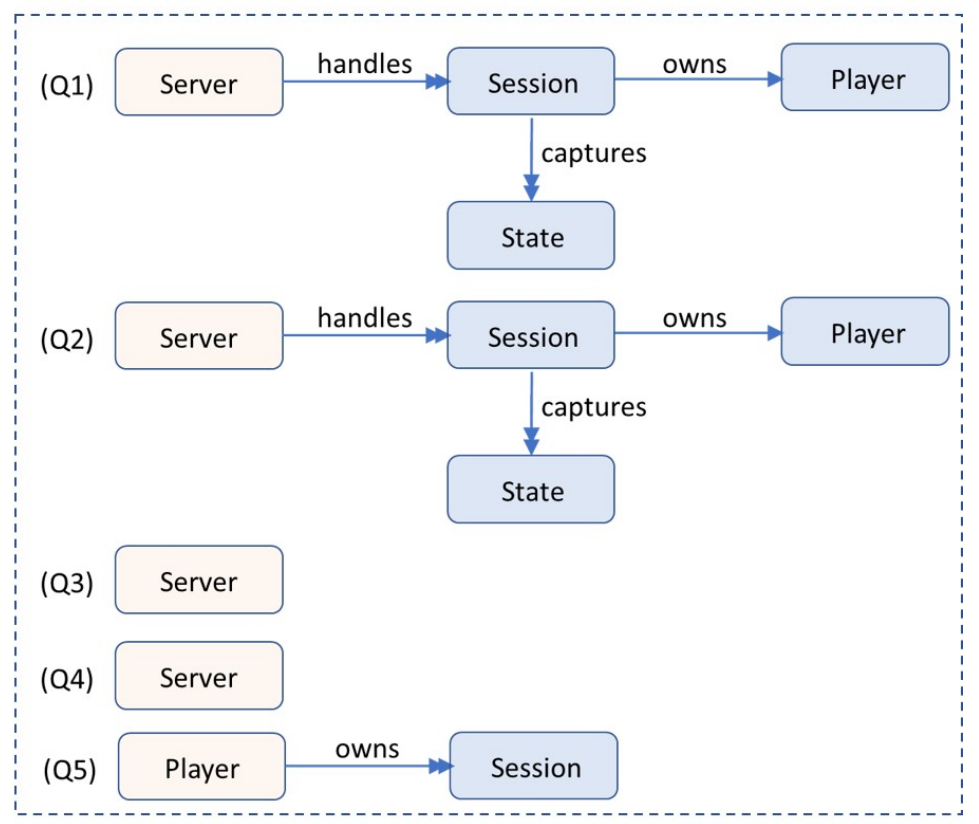

(b)

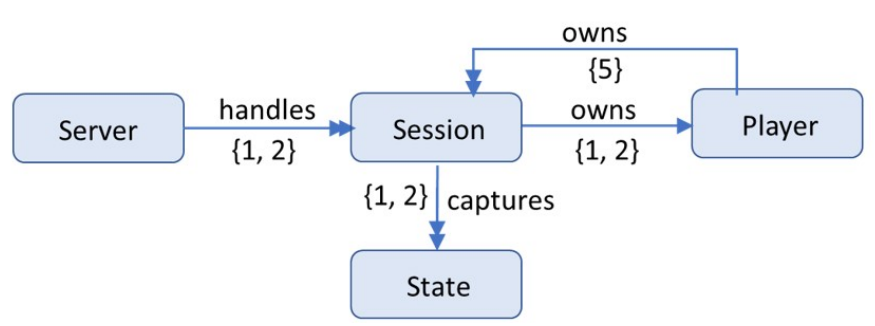

Fig. 5.10. (a) the paths of queries in the workload of the easy anti cheat; (b) the corresponding QPG

\subsection{Traversal-oriented partitioner evaluation}

We have implemented WASP as our traversal-oriented partitioner to evaluate the vertex reassignment presented in Section. It is available under a free license in an external repository ${ }^{3}$. In more detail, MPICH-3.1.4 library and ZeroMQ ${ }^{4}$ sockets are used for communication across nodes. In addition, Hiredis ${ }^{5}$ that is an official $\mathrm{C}$ client library is used for connecting to Redis KV stores. WASP is deployed on a cluster

\footnotetext{
3 https://github.com/alidavoudian/WASP-Graph-Partitioner/

4 http://zeromq.org

$5 \quad$ https://github.com/redis/hiredis
} 
(a)

\begin{tabular}{|c|c|c|c|c|c|}
\hline $\begin{array}{l}\text { QPG } \\
\text { node }\end{array}$ & $\begin{array}{c}\text { Scalar } \\
\text { properties }\end{array}$ & $\begin{array}{c}\text { Key } \\
\text { properties }\end{array}$ & $\begin{array}{l}\text { Access } \\
\text { point }\end{array}$ & $\begin{array}{c}\text { Query } \\
\text { numbers }\end{array}$ & $\begin{array}{c}\text { Out } \\
\text { neighbors }\end{array}$ \\
\hline Server & $\begin{array}{c}\text { Id/INT, name/STRING, } \\
\text { IP/STRING }\end{array}$ & id & Yes & $1,2,3,4$ & Session \\
\hline Session & $\begin{array}{c}\text { Id/INT, } \\
\text { startingTime/STRING, } \\
\text { endingTime/STRING }\end{array}$ & id & No & - & State, Player \\
\hline State & $\begin{array}{l}\text { Id/INT, posX/INT, } \\
\text { posY/INT, posZ/INT, } \\
\text { timestamp/INT }\end{array}$ & id & No & - & - \\
\hline Player & $\begin{array}{l}\text { Id/INT, name/STRING, } \\
\text { flags/STRING, } \\
\text { isAdmin/INT }\end{array}$ & id & Yes & 5 & Session \\
\hline
\end{tabular}

(b)

\begin{tabular}{|c|c|c|}
\hline QPG edge & $\begin{array}{c}\text { Query } \\
\text { numbers }\end{array}$ & Cardinality \\
\hline <Server, Session>/handles & 1,2 & $*$ \\
\hline <Session, State>/captures & 1,2 & $*$ \\
\hline <Session, Player>/owns & 1,2 & 1 \\
\hline <Player, Session>/owns & 5 & $*$ \\
\hline
\end{tabular}

(c)

\begin{tabular}{|c|c|c|c|l|}
\hline $\begin{array}{c}\text { Query } \\
\text { No }\end{array}$ & $\begin{array}{c}\text { Equality } \\
\text { properties }\end{array}$ & $\begin{array}{c}\text { Inequality } \\
\text { properties }\end{array}$ & $\begin{array}{c}\text { Ordering } \\
\text { properties }\end{array}$ & \multicolumn{1}{c|}{$\begin{array}{c}\text { Projection/aggregation } \\
\text { properties }\end{array}$} \\
\hline 1 & $\begin{array}{c}\text { Server.id, } \\
\text { Player.id }\end{array}$ & - & State.timestamp & Server.id, Session.id, Player.id, State.id, State.posX, State.posY, State.posZ, State.timestamp \\
\hline 2 & Server.id & - & $\begin{array}{c}\text { Player.id, } \\
\text { State.timestamp }\end{array}$ & Server.id, Session.id, State.id, State.posX, State.posY, State.posZ, State.timestamp \\
\hline 3 & Server.id & - & - & Server.id, Server.name, Server.IP \\
\hline 4 & Server.id & - & - & Server.id \\
\hline 5 & Player.id & - & - & Session.id \\
\hline
\end{tabular}

Table 5.4. (a) specification of QPG nodes, (b) QPG edges and (c) QPG queries in the the easy anti cheat
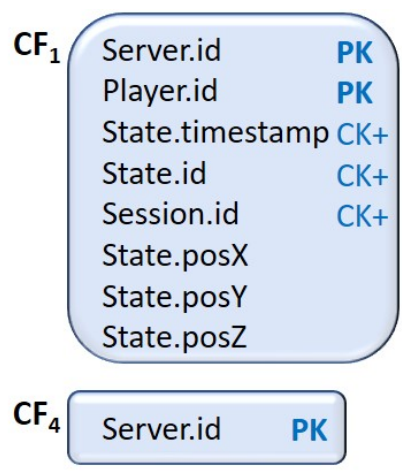

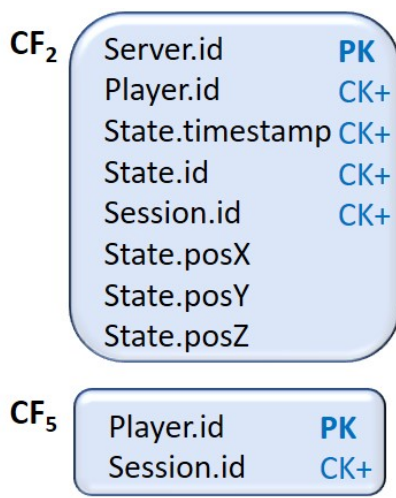

Fig. 5.11. The resulting CF schemas of mapping QPG in the the easy anti cheat

with 10 homogeneous nodes connected in a peer-to-peer fashion, where each node has 48GB of RAM, 16 quad-core CPUs of 2.4GHz, 160GB SATA HDD, and runs Debian 


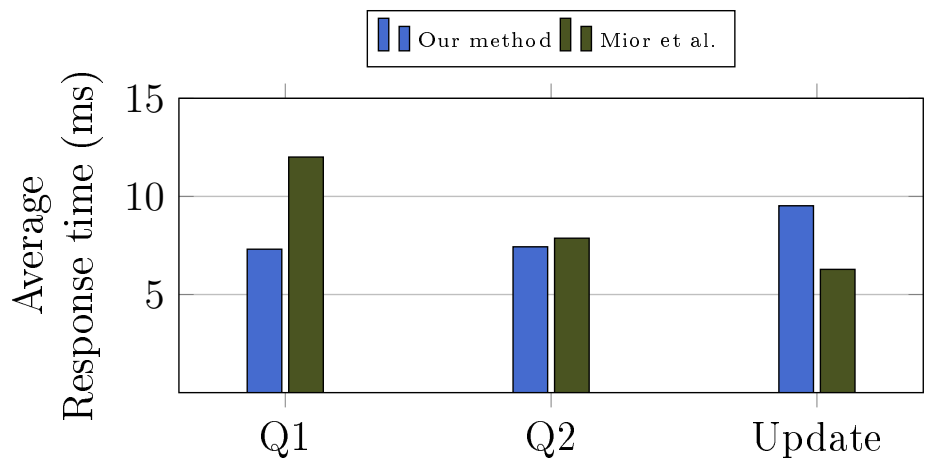

Fig. 5.12. Response times in our design vs. Mior et al.

Linux 6.0.6. 
Table 5.5. Dataset statistics in millions (M)

\begin{tabular}{llll}
\hline Dataset & \#Vertices & \#Edges & Avg./Max. degree \\
\hline WatDiv-1B [125] & $97 \mathrm{M}$ & $1092 \mathrm{M}$ & $24 / 190 \mathrm{~K}$ \\
\hline LUBM-10240 [126] & $332 \mathrm{M}$ & $1367 \mathrm{M}$ & $17 / 4.3 \mathrm{~K}$ \\
\hline DBpedia [127] & $14 \mathrm{M}$ & $1096 \mathrm{M}$ & $35 / 630 \mathrm{~K}$ \\
\hline
\end{tabular}

\subsubsection{Graph datasets and workloads}

We have conducted our experiments using three synthetic and real datasets (see Table 5.5) as well as six workloads over the datasets. WatDiv [125] and LUBM [126] are two synthetic property graph benchmarks whereby we create two datasets, each of which contains over 1 billion edges. We use their query template generators to generate 20 and 14 basic templates or patterns, respectively. We then instantiate $1 K$ queries for each template. Accordingly, we create two workloads for WatDiv, namely WatDiv-SW and WatDiv-DW, each of which contains a total of $20 K$ queries. The former simulates a static workload by shuffling the queries of all patterns with no fluctuating frequency. The latter simulates a dynamic workload by the consecutive execution of queries of the same pattern. Similarly, we create $L U B M-S W$ and $L U B M$ $D W$ workloads for LUBM, each of which contains a total of $14 K$ queries.

As both WatDiv and LUBM are synthetic, we also use DBpedia [127] as a real property graph dataset extracted from Wikipedia by crowdsourcing. We extract 44 query templates from the DBpedia query log [33], which spans from April 12, 2014, to October 16, 2014, recording over 1.7 million query requests. In order to extract query templates, we randomly select one-day queries from the log and transform them into a parameterized form [128]. We used the query log as it is also used by the other state-of-the-art graph partitioners, which in turn results in a fair comparison. We remove the queries with parse error or runtime error, and there remain 1.2 million 
queries in the end. As before, we create two workloads, namely DBpedia-SW and DBpedia- $D W$, each of which contains a total of $44 K$ queries. Note that most of the queries in the DBpedia query log consist of only one or a few triple patterns, so the templates of DBpedia are much simpler than the other datasets.

\subsubsection{Competitors}

Regarding Table 4.1, we compare WASP against Hermes [22] that is the only strategy with no prior knowledge of workloads. We also compare against the Peng et al. method [27] as a representative of strategies that are based on knowing a priori workload. It has already shown to have a faster query response time against WARP [25] and Partout [26].

\subsubsection{Partition quality}

We first study the IPT ratio achieved by the three partitioners, regarding the six static and dynamic workloads. In Figure 5.13(a), the X-axis shows the WatDiv-SW query stream in 10 units, each of which contains $2 K$ queries. The Y-axis indicates the IPT ratio achieved by executing the queries of each unit. The diagram shows a gradual decrease of IPT ratio in both Hermes and WASP as they gradually improve data access locality via their vertex reassignment strategies. However, since Hermes does not consider the weight of active edges, the corresponding decreasing rate is lower than WASP. On the other hand, despite WASP and Hermes that initially partition the graph dataset via the simple hashing strategy, the Peng et al. method [27] partitions the whole graph dataset assuming a priori knowledge of the WatDiv-SW workload. Therefore, the diagram shows an almost steady IPT ratio less than 0.1 , as the Peng et al. method has already assigned the matches of each frequent pattern to the same partition. 
On the contrary, regarding the WatDiv-DW query stream as shown in Figures 5.13(b), the IPT ratio in the Peng et al. method is very high (more than 0.8). In more detail, it partitions the graph dataset based on the frequent query patterns in the first workload unit, assuming a priori knowledge of the unit. However, the existing frequent query patterns change in subsequent units which results in a severe increase in IPT ratio as the Peng et al. method is not workload adaptive. On the other hand, WASP and Hermes adapt themselves to the WatDiv-DW query stream. During the workload, the IPT ratio in WASP fluctuates approximately between 0.3 to 0.4. However, Hermes acts in an upper bound of ratios between 0.6 to 0.7 , as it takes into account a uniform frequency of edge traversals despite the non-uniform ones in real-world scenarios. Similar reasoning can be used to justify the results obtained for LUBM and DBpedia datasets. As Figure 5.13(c) shows, after executing the $L U B M-S W$ query stream, WASP achieves a better IPT ratio (almost 0.27) compared to the one in WatDiv-SW (almost 0.43 in Figure 5.13(a)). This is due to the existence of more complex queries with long paths in LUBM than WatDiv. In more detail, more complex queries cause more edge traversals, which results in the identification of hot edges and collocating their endpoint vertices by WASP. On the other hand, as Figure 5.13(e) shows, the behavior of WASP and Hermes are closer to each other compared to Figures 5.13(a) and (c). This is because of simple short path queries in DBpedia.

Note that the improvement of the IPT ratio does not necessarily result in the improvement of query performance unless there is a balanced load distribution [117]. Therefore, we study the "computational" load balance achieved by the three partitioners. In this respect, although Hermes and the Peng et al. method exploit the existing workload to achieve a balanced load distribution, they perform poorly in WatDiv and DBpedia as all edges of very high-degree vertices are grouped together. This causes a subset of machines to be computationally overloaded by active vertices 


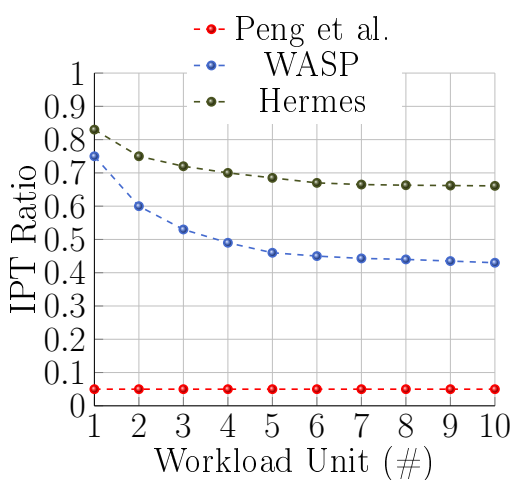

(a) WatDiv-SW (20K)

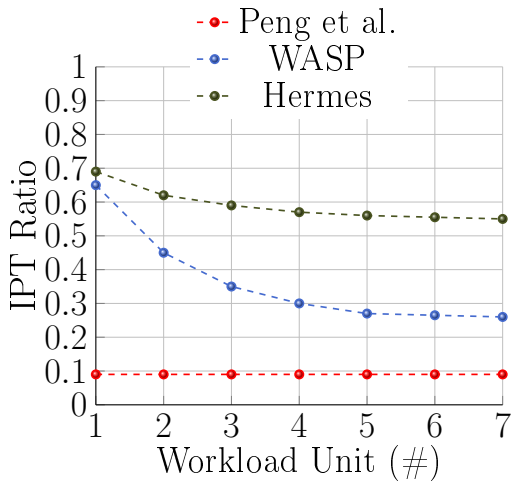

(c) LUBM-SW (14K)

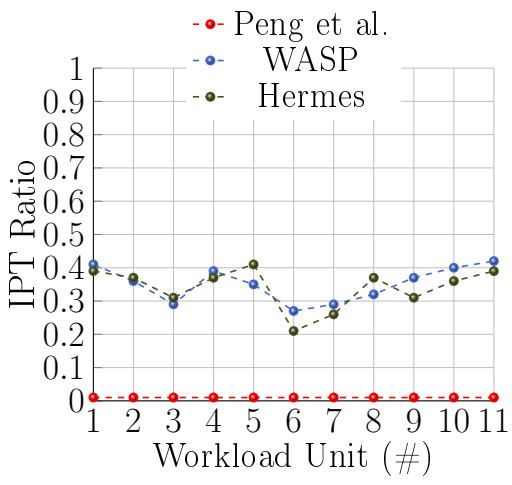

(e) DBpedia-SW (44K)

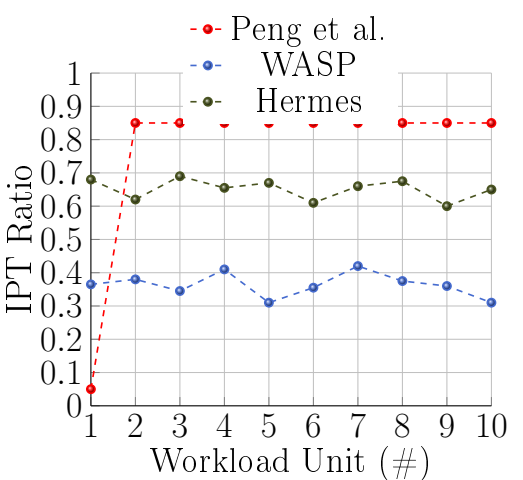

(b) WatDiv-DW (20K)

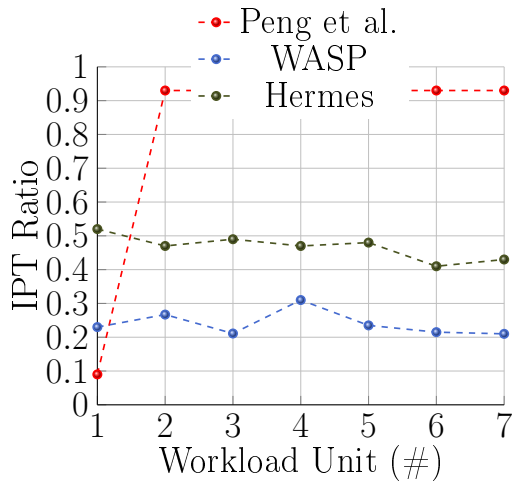

(d) LUBM-DW (14K)

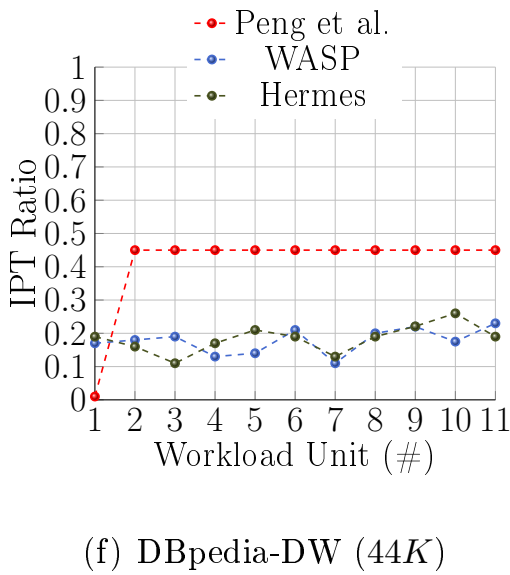

Fig. 5.13. IPT ratio regarding the six workloads 
with a very high degree. On the other hand, WASP splits such vertices (see Section 4.2.3). Accordingly, Fig. 5.14 shows the load imbalance factor $(\phi)$ achieved by the partitioning strategies on the three static workloads. As shown, WASP achieves a better load imbalance than the others in all the workloads.

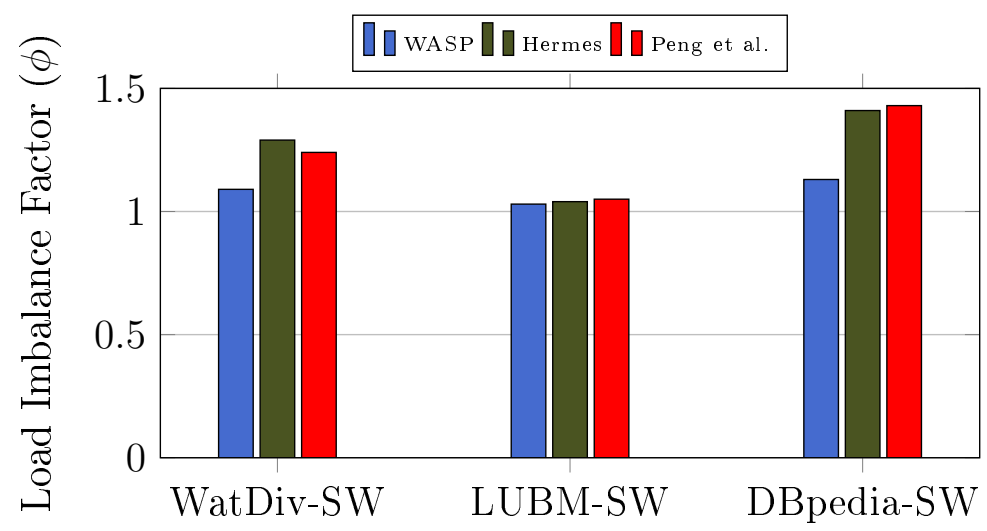

Fig. 5.14. Load imbalance factor regarding the three static workloads

\subsubsection{Query performance}

Table 5.6 shows the execution time of the six aforementioned static and dynamic workloads, regarding the three partitioning strategies. Unlike the Peng et al. method which initially mines frequent query patterns from the static workloads, WASP and Hermes use initial hash partitioning that mounts communication on complex queries. This results in better performance of the Peng et al. method compared to Hermes and WASP, regarding WatDiv and LUBM static workloads. However, WASP achieves a lower execution time than Peng et al. regarding DBpedia-SW. This is because of either the low impact of IPT ratio on query performance due to the simple short path queries in DBpedia or the high impact of load imbalance factor on query performance regardless of the complexity of queries. On the other hand, unlike WASP and Hermes, the Peng et al. method does not adapt to changes in frequent query patterns. This incurs high communication costs for the dynamic workloads and results in achieving 
Table 5.6. Workload runtime (sec)

\begin{tabular}{lllllll}
\hline Method & WatDiv-SW & LUBM-SW & DBpedia-SW & WatDiv-DW & LUBM-DW & DBpedia-DW \\
\hline Peng et al. & 924 & 830 & 435 & 1332 & 1396 & 515 \\
\hline Hermes & 1210 & 1153 & 481 & 1244 & 1136 & 487 \\
\hline WASP & 987 & 1089 & 377 & 916 & 843 & 347 \\
\hline
\end{tabular}

higher workload execution times in the Peng et al. method compared to the other strategies.

\subsubsection{Reassignment threshold sensitivity analysis}

The reassignment threshold controls the triggering of the vertex reassignment process. Consequently, it influences the edge-cut ratio and the number of vertex reassignments in the system. In this experiment, we conduct an empirical sensitivity analysis to select the reassignment threshold value regarding the aforementioned static workloads. We execute each workload regarding a wide range of possible reassignment threshold values from 1 to 30 as load execution times get almost steady regarding the threshold value 30. The edge-cut ratio, the number of vertex reassignments and the resulting workload execution times are shown in Fig. 5.15(a)-(c), respectively. As Fig. 5.15(a) and (b) show, there is a contrast between edge-cut ratio and the number of vertex reassignments. This necessitates to make a balance between these factors in order to decrease load execution times. We observe that WatDiv is very sensitive to slight changes in the reassignment threshold because of the complexity of its queries. As the reassignment threshold increases, the reassignment of active vertices is delayed causing more queries to be executed with communication. On the other hand, by decreasing the reassignment threshold, more vertices are reassigned causing more overhead of data/metadata maintenance. As it can be seen, DBpedia is not as sensitive to this range of reassignment thresholds because most of its queries are simple 


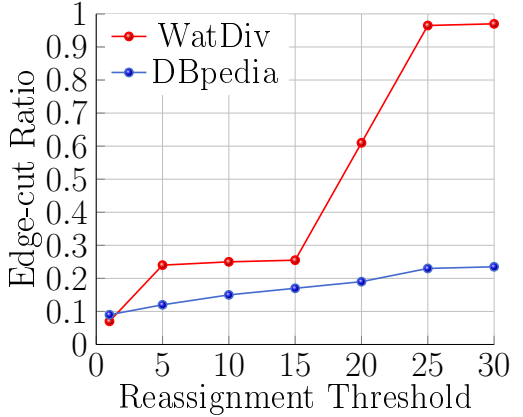

(a)

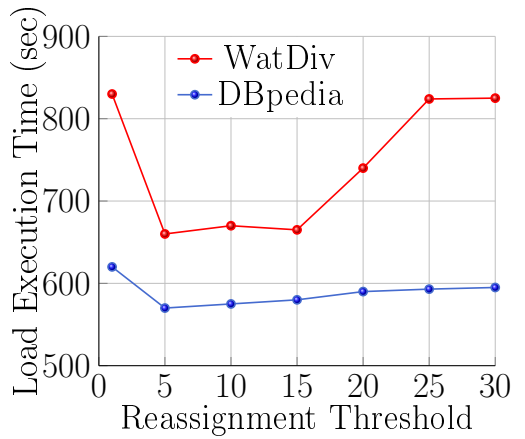

(c)

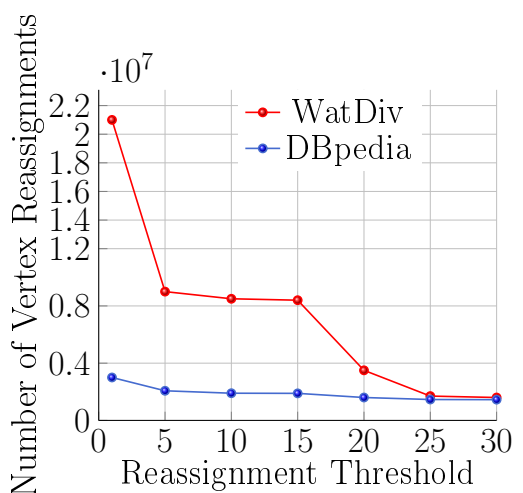

(b)

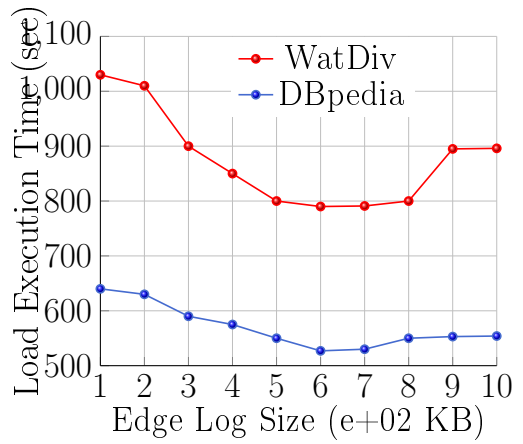

(d)

Fig. 5.15. Sensitivity analysis of reassignment threshold and edge log size 
short path queries that are processed with low or no communication. As the both workloads show, either increasing or decreasing the reassignment threshold may impact the efficiency and throughput of query processing. In all our experiments, we use a reassignment threshold of 10; this results in a good balance between the edge-cut ratio and the number of vertex reassignments. We plan to study the autotuning of this parameter in the future.

\subsubsection{Edge log size sensitivity analysis}

Edge log size or $\Delta$ affects the lifespan of active edges and the quality of partitions. This incurs choosing a proper log size value. By increasing $\Delta$, both recent and old active edges are considered in partitioning. This decreases the throughput due to an improper load balance. More precisely, the weights of endpoint vertices associated with old active edges are still considered in calculating their hosting nodes' weights. On the other hand, by decreasing $\Delta$, only a subset of recent active edges are taken into account in partitioning. This decreases the efficiency of queries via increasing edge-cut ratio as the endpoint vertices of some recent active edges are not collocated. Hence, decreasing or increasing $\Delta$ can impact the workload execution time. In this experiment, we conduct an empirical sensitivity analysis to select a proper edge log size based on the workload execution time, regarding WatDiv-SW and DBpedia-SW workloads. As typical workloads access only a small fraction of the whole graph, we execute each workload regarding a range of log sizes from $100 K$ to $1000 K$ as workload execution times get almost steady regarding the lower and upper bounds. Workload execution times are shown in Fig. 5.15(d). In all subsequent experiments, we use an

edge log size of $700 K$; this results in a high throughput and query efficiency. We plan to study the autotuning of reassignment threshold and edge log size in the future. 


\subsubsection{Splitting threshold sensitivity analysis}

The splitting threshold controls the cutting up of high-degree vertices. However, despite alleviating the computational load imbalance, splitting the edges leads to the replication of high-degree vertices. This in turn introduces extra network overhead to retrieve data from remote nodes. Therefore, it is important to find the best splitting threshold to make a trade-off between the computational load balance and the average number of replicas. In this experiment, we conduct an empirical sensitivity analysis to select the splitting threshold value regarding the WatDiv and DBpedia datasets in a cluster of 10 nodes.

The result (Fig. 5.16) shows that by increasing the splitting threshold, fewer vertices get replicated causing a sharp decrease in the average number of replicas. However, in both datasets, the average number of replicas is almost insensitive to the splitting thresholds larger than 100 that is selected as the splitting threshold in our experiments.

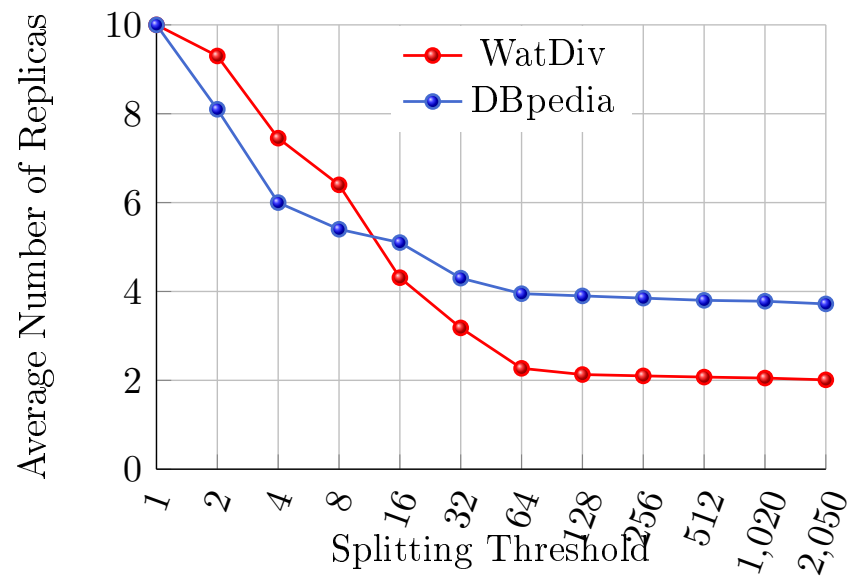

Fig. 5.16. Average number of replicas under different splitting thresholds 


\subsubsection{Workload evolution}

In this experiment, we use the aforementioned dynamic workloads to investigate the impact of changing frequent query patterns on our partitioning strategy. Regarding the WatDiv-DW workload, Fig. 5.17(a) shows the cumulative time as the execution progresses using our method with and without the adaptivity feature. After every sequence of $1 \mathrm{~K}$ query executions, the pattern of queries changes. Without adaptivity, the cumulative time increases sharply. On the other hand, our method adapts to the dynamic workload with little overhead causing the cumulative time to drop significantly by almost 6 times. Once our method starts adapting, most of future queries are solved with less communication. The same behavior is observed for the DBpedia-DW workload (see Fig. 5.17(b)).

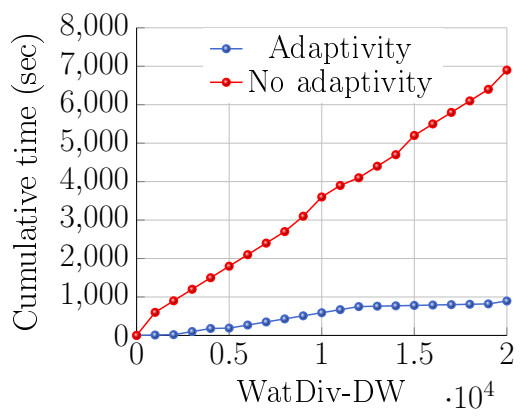

(a)

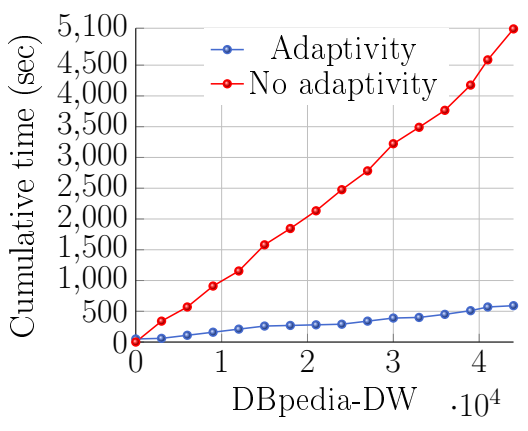

(b)

Fig. 5.17. WASP adaptivity to dynamic workloads

\subsubsection{Data scalability}

In this experiment, we investigate the impact of dataset size on our fragmentation strategies. We use the WatDiv benchmark data generator to generate four datasets: WatDiv-1M, WatDiv-10M, WatDiv-100M, WatDiv-1B, varying from 1 million to 1 
billion triples, respectively. Regarding the WatDiv-SW workload, Fig. 5.18(a) and (b) show the performance and throughput of our partitioning method with and without the adaptivity feature by increasing the dataset size. Generally, as the size of RDF datasets gets larger, the average response time per query increases and the number of queries answered in 1 minute decreases accordingly. However, the rates of increase and decrease in the method with adaptivity are gradual compared to the method without adaptivity, and we can say that the query performance and throughput are scalable with RDF graph sizes.

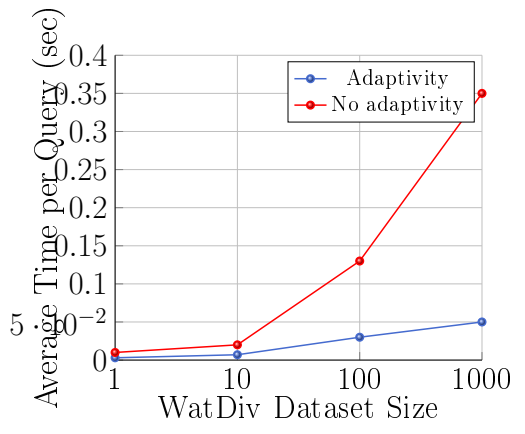

(a) Performance

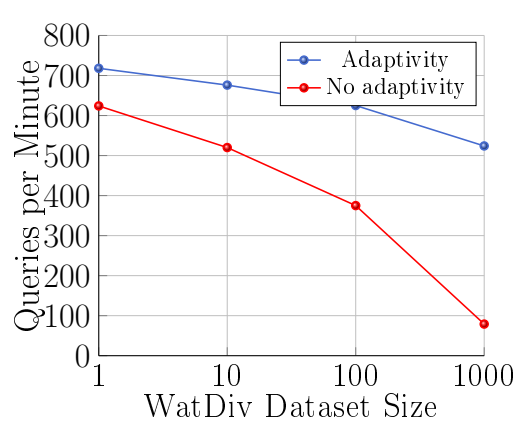

(b) Throughput

Fig. 5.18. WASP scalability regarding the WatDiv dataset

\subsection{Summary}

The main methods and algorithms introduced in this thesis are empirically validated and evaluated, using proof of concept implementations and a multitude of real-world datasets. We conducted an experiment using 3 typical case studies from the literature of NoSQL to evaluate the quality of database schemas generated using our key-oriented partitioner. The results obtained from this experiment demonstrate that partitioner preserves not only the expressiveness of QPG to model different 
aggregate-oriented NoSQL databases, but also the quality of the design by making a trade-off between the cost of read operations with the cost of either write operations or storage. We also conducted multiple experiments using 3 synthetic and realworld graph datasets to evaluate the adaptivity, performance and scalability of our traversal-oriented partitioner. Our experiments show that WASP achieves a better query performance against state-of-the-art graph partitioners, especially in dynamic workloads. 


\section{Chapter 6}

\section{Conclusion and Future Work}

Due to the scalability problems in traditional relational database systems, a variety of NoSQL stores have emerged over the last decade to deal with big data. The lack of standard processes for designing NoSQL database schemas has led to the proposal of several recent methods. However, they provide various conceptual modeling notations and mainly target a particular NoSQL data model that cause extra effort for designers when switching from one data model to another. In addition, by providing just a set of guidelines and heuristics for the design process, many methods have to be applied manually which is an error-prone and time-consuming process. To deal with these limitations, we present a key-oriented designer and partitioner for designing key-value, wide-column, and document NoSQL database schemas from the same conceptual model. It first generates a generic NoSQL logical schema from the conceptual model and workload of the system. Then it converts the generic schema to the schemas of targeted NoSQL data models regarding their important features and design trade-offs between the read query performance and storage overhead or consistency maintenance.

On the other hand, streaming graph partitioning methods have recently gained attention due to their ability to scale to very large graphs with limited resources. However, many such methods do not consider workload and graph characteristics. 
This may degrade the performance of queries by increasing inter-node communication and computational load imbalance. Moreover, existing workload-aware methods cannot consistently provide good performance as they do not consider dynamic workloads that keep emerging in graph applications. We address these issues by proposing a novel workload-adaptive streaming partitioner named WASP, that aims to achieve low-latency and high-throughput online graph queries. As each workload typically contains frequent query patterns, WASP exploits the existing workload to capture active vertices and edges which are frequently visited and traversed respectively. This information is used to heuristically improve the quality of partitions either by avoiding the concentration of active vertices in a few partitions proportional to their visit frequencies or by reducing the probability of the cut of active edges proportional to their traversal frequencies.

To demonstrate the usefulness of our partitioning methods and their roles in satisfying existing query requirements, we conducted various experiments on adaptivity, performance and scalability on various datasets. By evaluating the key-oriented partitioner using several typical case studies from the literature of NoSQL, we have concluded the expressiveness of QPG to model different aggregate-oriented NoSQL databases, the validity and quality of generated database schemas and the low computational cost of the method. In addition, the experiments show that WASP significantly reduces the number of inter-partition traversals during a workload. It is able to handle evolving workloads and graph topology while maintaining the quality of partitions over time.

Future work could take many directions. In the following subsections, we summarize the pressing ones.

- Lack of load balancing and adaptivity features. As explained in Section 2.2.2, a workload-driven key-based partitioner should take into account the adaptivity of 
existing partitions with the workload characteristics. However, there is no adaptivity feature and workload balance in our key-oriented partitioner.

- Lack of parameter autotuning. As explained in Section 4.2.3, WASP exploits hardwired thresholds for triggering vertex reassignment and splitting high-degree vertices. However, such parameters can be autotuned with different workload characteristics and query requirements.

- Lack of replication. Currently, there is no replication strategy in WASP regarding the storage overhead. However, a workload-driven replication can be exploited whereby the replication scheme of a vertex (i.e., how many replicas of the vertex are created, and to which partitions these replicas are allocated) dynamically changes based on the read/write frequencies of the vertex.

- Lack of pattern-based hot edge recognition. Currently, WASP needs to wait for edges of the input graph to get hot, in order to collocate their endpoint vertices. However, this collocation can be performed by taking into account the hot edges of a pattern graph made by combining query patterns in the existing workload. This collocation is lighter and faster. 


\section{List of References}

[1] A. Davoudian and M. Liu, "Big data systems: A software engineering perspective," ACM Computing Surveys (CSUR), vol. 53, no. 5, p. 110, 2020.

[2] T. Haerder and A. Reuter, "Principles of transaction-oriented database recovery," ACM computing surveys (CSUR), vol. 15, no. 4, pp. 287-317, 1983.

[3] J. Gray, P. Helland, P. O'Neil, and D. Shasha, "The dangers of replication and a solution," in ACM SIGMOD Record, vol. 25, pp. 173-182, ACM, 1996.

[4] P. Helland, "Life beyond distributed transactions: An apostate's opinion," in CIDR, pp. 132-141, 2007.

[5] J. B. Rothnie and N. Goodman, "A survey of research and development in distributed database management," in Proceedings of the 3rd International Conference on Very Large Databases, pp. 48-62, VLDB Endowment, 1977.

[6] A. Fox and E. A. Brewer, "Harvest, yield, and scalable tolerant systems," in Proceedings of the 7th Workshop on Hot Topics in Operating Systems, pp. 174178, IEEE, 1999.

[7] E. A. Brewer, "Towards robust distributed systems," in PODC, vol. 7, 2000.

[8] S. Gilbert and N. Lynch, "Brewer's conjecture and the feasibility of consistent, available, partition-tolerant web services," ACM SIGACT News, vol. 33, no. 2, pp. 51-59, 2002.

[9] E. Brewer, "CAP twelve years later: How the "rules" have changed," Computer, vol. 45, no. 2, pp. 23-29, 2012.

[10] A. Davoudian, L. Chen, and M. Liu, "A survey on NoSQL stores," ACM Computing Surveys (CSUR), vol. 51, no. 2, p. 40, 2018.

[11] R. Cattell, "Scalable SQL and NoSQL data stores," ACM SIGMOD Record, vol. 39, no. 4, pp. 12-27, 2011. 
[12] P. Helland, "Life beyond distributed transactions: An apostate's opinion," in CIDR, vol. 2007, pp. 132-141, 2007.

[13] E. E, Domain-driven design: Tacking complexity In the heart of software. Addison-Wesley Longman Publishing Co., Inc., Boston, MA, 2003.

[14] T. Vajk, P. Fehér, K. Fekete, and H. Charaf, "Denormalizing data into schemafree databases," in 4th International Conference on Cognitive Infocommunications, pp. 747-752, IEEE, 2013.

[15] V. C. Storey and I.-Y. Song, "Big data technologies and management: What conceptual modeling can do," Data $\&$ Knowledge Engineering, vol. 108, pp. 50$67,2017$.

[16] N. Bruno and S. Chaudhuri, "Constrained physical design tuning," VLDB Endowment, vol. 1, no. 1, pp. 4-15, 2008.

[17] S. Agrawal, S. Chaudhuri, L. Kollar, A. Marathe, V. Narasayya, and M. Syamala, "Database tuning advisor for Microsoft SQL server 2005," in ACM SIGMOD, pp. 930-932, 2005.

[18] S. Agrawal, E. Chu, and V. Narasayya, "Automatic physical design tuning: Workload as a sequence," in ACM SIGMOD, pp. 683-694, 2006.

[19] D. C. Zilio, J. Rao, S. Lightstone, G. Lohman, A. Storm, C. Garcia-Arellano, and S. Fadden, "Db2 design advisor: Integrated automatic physical database design," in 13th international conference on VLDB, pp. 1087-1097, 2004.

[20] J. Lu and I. Holubová, "Multi-model databases: A new journey to handle the variety of data," ACM Computing Surveys (CSUR), vol. 52, no. 3, pp. 1-38, 2019.

[21] U. Störl, M. Klettke, and S. Scherzinger, "NoSQL schema evolution and data migration: State-of-the-art and opportunities.," in EDBT, pp. 655-658, 2020.

[22] D. Nicoara, S. Kamali, K. Daudjee, and L. Chen, "Hermes: Dynamic partitioning for distributed social network graph databases," in Proceedings of the 18th EDBT International Conference on Extending Database Technology, 2015.

[23] H. Firth and P. Missier, "Workload-aware Streaming Graph Partitioning," in EDBT/ICDT Workshops, 2016. 
[24] H. Firth and P. Missier, "TAPER: query-aware partition-enhancement for large heterogenous graphs," Proceedings of the Distributed and Parallel Databases, vol. 35, no. 2, 2017.

[25] K. Hose and R. Schenkel, "WARP: Workload-aware replication and partitioning for RDF," in 29th International Conference on Data Engineering Workshops (ICDEW), pp. 1-6, IEEE, 2013.

[26] L. Galárraga, K. Hose, and R. Schenkel, "Partout: A distributed engine for efficient RDF processing," in Proceedings of the 23rd International Conference on World Wide Web, pp. 267-268, ACM, 2014.

[27] P. Peng, L. Zou, L. Chen, and D. Zhao, "Adaptive distributed rdf graph fragmentation and allocation based on query workload," IEEE Transactions on Knowledge and Data Engineering, vol. 31, no. 4, pp. 670-685, 2019.

[28] R. Harbi, I. Abdelaziz, P. Kalnis, N. Mamoulis, Y. Ebrahim, and M. Sahli, "Accelerating SPARQL queries by exploiting hash-based locality and adaptive partitioning," The VLDB Journal-The International Journal on Very Large Data Bases, vol. 25, no. 3, pp. 355-380, 2016.

[29] J. Huang and D. J. Abadi, "Leopard: Lightweight edge-oriented partitioning and replication for dynamic graphs," Proceedings of the VLDB Endowment, vol. 9, no. $7,2016$.

[30] D. Dai, W. Zhang, and Y. Chen, "IOGP: An incremental online graph partitioning algorithm for distributed graph databases," ACM Press, 2017.

[31] H. Firth, P. Missier, and J. Aiston, "Loom: Query-aware partitioning of online graphs," arXiv preprint arXiv:1711.06608, 2017.

[32] H. Pang, P. Gan, P. Yuan, H. Jin, and Q. Hua, "Partitioning large-scale property graph for efficient distributed query processing," in 21st International Conference on High Performance Computing and Communications, pp. 1643-1650, IEEE, 2019.

[33] M. Saleem, M. I. Ali, A. Hogan, Q. Mehmood, and A.-C. N. Ngomo, "LSQ: the linked sparql queries dataset," in International semantic web conference, pp. 261-269, Springer, 2015.

[34] K. Peffers, T. Tuunanen, M. A. Rothenberger, and S. Chatterjee, "A design science research methodology for information systems research," Journal of management information systems, vol. 24, no. 3, pp. 45-77, 2007. 
[35] J. L. Carlson, Redis in action. Manning Publications Co., 2013.

[36] M. Stonebraker, "NewSQL: An alternative to NoSQL and old SQL for new OLTP apps.," Communications of the ACM, pp. 07-06, 2012.

[37] A. Silberschatz, H. F. Korth, and S. Sudarshan, "Data models," ACM Computing Surveys (CSUR), vol. 28, no. 1, pp. 105-108, 1996.

[38] M. Massé, REST API design rulebook: Designing consistent RESTful Web Service Interfaces. O’Reilly, 2012.

[39] A. Lakshman and P. Malik, "Cassandra: A decentralized structured storage system," ACM SIGOPS Operating Systems Review, vol. 44, no. 2, pp. 35-40, 2010.

[40] F. Chang, J. Dean, S. Ghemawat, W. C. Hsieh, D. A. Wallach, M. Burrows, T. Chandra, A. Fikes, and R. E. Gruber, "BigTable: A distributed storage system for structured data," ACM Transactions on Computer Systems (TOCS), vol. 26, no. 2, 2008.

[41] P. O’Neil, E. Cheng, D. Gawlick, and E. O’Neil, “The log-structured merge-tree (LSM-tree)," Acta Informatica, vol. 33, no. 4, pp. 351-385, 1996.

[42] J. Dean and S. Ghemawat, "MapReduce: Simplified data processing on large clusters," Communications of the ACM, vol. 51, no. 1, pp. 107-113, 2008.

[43] J. C. Corbett, J. Dean, M. Epstein, A. Fikes, C. Frost, J. J. Furman, S. Ghemawat, A. Gubarev, C. Heiser, P. Hochschild, and others, "Spanner: Google's globally distributed database," ACM Transactions on Computer Systems (TOCS), vol. 31, no. 3, p. 8, 2013.

[44] M. Stonebraker, "Why enterprises are uninterested in NoSQL," 2011.

[45] D. Maier, "Why database languages are a bad idea," in Proceedings of the International Workshop on Database Programming Languages, pp. 277-287, 1989.

[46] C. Zaniolo, H. A. t Kaci, D. Beech, S. Cammarata, L. Kerschberg, and D. Maier, "Object-oriented database and knowledge systems," tech. rep., Technical Report DB-038-85, MCC, 1985.

[47] K. Lee and L. Liu, "Scaling queries over big RDF graphs with semantic hash partitioning," vol. 6, no. 14, pp. 1894-1905, 2013. 
[48] T. Berners-Lee, J. Hendler, O. Lassila, and others, "The semantic web," Scientific american, vol. 284, no. 5, pp. 28-37, 2001.

[49] A. Schenker, A. Kandel, H. Bunke, and M. Last, Graph-theoretic techniques for web content mining, vol. 62. World Scientific, 2005.

[50] D. Knisley and J. Knisley, "Graph theoretic models in chemistry and molecular biology," HANDBOOK OF APPLIED ALGORITHMS, p. 85, 2007.

[51] B. A. Eckman and P. G. Brown, "Graph data management for molecular and cell biology," IBM journal of research and development, vol. 50, no. 6, pp. 545-560, 2006.

[52] A. Bretto, Hypergraph Theory. Mathematical Engineering, Springer International Publishing, 2013.

[53] S. Y. Lee, T. W. Ling, and H.-G. Li, "Hierarchical compact cube for range-max queries," in $V L D B$, pp. 232-241, 2000.

[54] Q. Luo and J. F. Naughton, "Form-based proxy caching for database-backed web sites," in $V L D B$, pp. 191-200, 2001.

[55] B. Knutsson, H. Lu, W. Xu, and B. Hopkins, "Peer-to-peer support for massively multiplayer games," in 23rd Joint Conference of the IEEE Computer and Communications Societies (INFOCOM'2004), vol. 1, IEEE, 2004.

[56] B. F. Cooper, R. Ramakrishnan, U. Srivastava, A. Silberstein, P. Bohannon, H.-A. Jacobsen, N. Puz, D. Weaver, and R. Yerneni, "PNUTS: Yahoo!'s hosted data serving platform," Proceedings of the VLDB Endowment, vol. 1, no. 2, pp. 1277-1288, 2008.

[57] J. Rao, E. J. Shekita, and S. Tata, "Using paxos to build a scalable, consistent, and highly available datastore," Proceedings of the VLDB Endowment, vol. 4, no. 4, pp. 243-254, 2011.

[58] G. DeCandia, D. Hastorun, M. Jampani, G. Kakulapati, A. Lakshman, A. Pilchin, S. Sivasubramanian, P. Vosshall, and W. Vogels, "Dynamo: amazon's highly available key-value store," in ACM SIGOPS Operating Systems Review, vol. 41, pp. 205-220, ACM, 2007.

[59] D. Karger, E. Lehman, T. Leighton, R. Panigrahy, M. Levine, and D. Lewin, "Consistent hashing and random trees: Distributed caching protocols for relieving hot spots on the World Wide Web," in Proceedings of the 29th annual ACM Symposium on Theory of Computing, pp. 654-663, ACM, 1997. 
[60] D. M. Lewin, "Consistent hashing and random trees: Algorithms for caching in distributed networks," 1998.

[61] I. Stoica, R. Morris, D. Karger, M. F. Kaashoek, and H. Balakrishnan, "Chord: A scalable peer-to-peer lookup service for internet applications," $A C M S I G$ COMM Computer Communication Review, vol. 31, no. 4, pp. 149-160, 2001.

[62] F. Dabek, M. F. Kaashoek, D. Karger, R. Morris, and I. Stoica, "Wide-area cooperative storage with CFS," in ACM SIGOPS Operating Systems Review, vol. 35, pp. 202-215, ACM, 2001.

[63] M. Hogqvist, S. Haridi, N. Kruber, A. Reinefeld, and T. Schutt, "Using global information for load balancing in DHTs," in 2nd IEEE International Conference on Self-Adaptive and Self-Organizing Systems Workshops (SASOW' 2008)., pp. 236-241, IEEE, 2008.

[64] B. Shao, H. Wang, and Y. Li, "Trinity: A distributed graph engine on a memory cloud," in International Conference on Management of Data, 2013.

[65] S. Ratnasamy, P. Francis, M. Handley, R. Karp, and S. Shenker, "A scalable content-addressable network," vol. 31, ACM SIGCOMM, 2001.

[66] A. Rowstron and P. Druschel, "Pastry: Scalable, decentralized object location, and routing for large-scale peer-to-peer systems," in Middleware 2001, pp. 329350, Springer, 2001.

[67] Y. Kwon, M. Balazinska, B. Howe, and J. Rolia, "Skew-resistant parallel processing of feature-extracting scientific user-defined functions," in Proceedings of the 1st ACM symposium on Cloud computing, pp. 75-86, ACM, 2010.

[68] M. Stonebraker, J. Duggan, L. Battle, and O. Papaemmanouil, "SciDB DBMS research at MIT," IEEE Database Eng. Bull., vol. 36, no. 4, pp. 21-30, 2013.

[69] B. Fleisch and G. Popek, "Mirage: A coherent distributed shared memory design," in Proceedings of the 14th ACM Symposium on Operating System Principles, pp. 211-223, 1989.

[70] A. Rao, K. Lakshminarayanan, S. Surana, R. Karp, and I. Stoica, "Load balancing in structured P2p systems," in Peer-to-Peer Systems II, pp. 68-79, Springer, 2003. 
[71] B. Godfrey, K. Lakshminarayanan, S. Surana, R. Karp, and I. Stoica, "Load balancing in dynamic structured P2p systems," in 23rd Annual Joint Conference of the IEEE Computer and Communications Societies (INFOCOM'2004), vol. 4, pp. 2253-2262, IEEE, 2004.

[72] C. Chen and K.-C. Tsai, "The server reassignment problem for load balancing in structured P2p systems," IEEE Transactions on Parallel and Distributed Systems, vol. 19, no. 2, pp. 234-246, 2008.

[73] M. R. Garey, D. S. Johnson, and L. Stockmeyer, "Some simplified NP-complete problems," in Proceedings of the 6th annual ACM Symposium on Theory of Computing, 1974.

[74] M. Faloutsos, P. Faloutsos, and C. Faloutsos, "On power-law relationships of the internet topology," in Proceedings of the ACM SIGCOMM Special Interest Group on Data Communication, vol. 29, 1999.

[75] J. E. Gonzalez, Y. Low, H. Gu, D. Bickson, and C. Guestrin, "PowerGraph: Distributed graph-parallel computation on natural graphs," in Proceedings of the 10th USENIX OSDI Conference on Operating Systems Design and Implementation, 2012.

[76] G. Karypis and V. Kumar, "A fast and high quality multilevel scheme for partitioning irregular graphs," SIAM Journal on Scientific Computing, vol. 20, no. 1, 1998.

[77] G. Karypis and V. Kumar, "Parallel multilevel series k-way partitioning scheme for irregular graphs," Siam Review, vol. 41, no. 2, 1999.

[78] C. Tsourakakis, C. Gkantsidis, B. Radunovic, and M. Vojnovic, "Fennel: Streaming graph partitioning for massive scale graphs," in Proceedings of the 7th ACM international conference on Web search and data mining, 2014.

[79] J. Nishimura and J. Ugander, "Restreaming graph partitioning: Simple versatile algorithms for advanced balancing," in Proceedings of the 19th ACM SIGKDD International Conference on Knowledge Discovery and Data Mining, 2013.

[80] J. Ugander and L. Backstrom, "Balanced label propagation for partitioning massive graphs," in Proceedings of the 6th ACM international conference on Web search and data mining, 2013.

[81] Oracle Inc, "Oracle NoSQL Database," [Online]. 
[82] Apache Inc, "What is Cassandra?," [Online].

[83] MongoDB Inc, "The database for modern applications," [Online].

[84] Oracle Inc, "Interface TableAPI," [Online].

[85] MongoDB Inc, "BSON," [Online].

[86] JSON Organization, "JSON Schema," [Online].

[87] C. Li, "Transforming relational database into hbase: A case study," in International conference on software engineering and service sciences, pp. 683-687, IEEE, 2010.

[88] P. Atzeni, F. Bugiotti, L. Cabibbo, and R. Torlone, "Data modeling in the NoSQL world," Computer Standards \& Interfaces, vol. 67, p. 103149, 2020.

[89] A. Chebotko, A. Kashlev, and S. Lu, "A big data modeling methodology for Apache Cassandra," in International Congress on big data, pp. 238-245, IEEE, 2015 .

[90] M. Lawley and R. W. Topor, "A query language for eer schemas.," in Australasian Database Conference, pp. 292-304, 1994.

[91] M. J. Mior, K. Salem, A. Aboulnaga, and R. Liu, "Nose: Schema design for NoSQL applications," IEEE Transactions on Knowledge and Data Engineering, vol. 29, no. 10, pp. 2275-2289, 2017.

[92] C. de Lima and R. S. Mello, "On proposing and evaluating a nosql document database logical approach," International Journal of Web Information Systems, vol. 12, no. 4, pp. 398-417, 2016.

[93] R. Schroeder, D. Duarte, and R. S. Mello, "A workload-aware approach for optimizing the xml schema design trade-off," in 13th International Conference on Information Integration and Web-based Applications and Services, pp. 12-19, 2011.

[94] Apache Inc, "Welcome to Apache HBase," [Online].

[95] W. Winston, Introduction to Mathematical Programming: Applications and Algorithms, Duxbury,(2002). Duxbury, 2002.

[96] T. Halpin and T. Morgan, Information modeling and relational databases. Morgan Kaufmann, 2010. 
[97] O. G. Tsatalos, M. H. Solomon, and Y. E. Ioannidis, "The gmap: A versatile tool for physical data independence," The International Journal on Very Large Data Bases (VLDB), vol. 5, no. 2, pp. 101-118, 1996.

[98] MongoDB Inc, "Document Schemas," [Online].

[99] C. Curino, E. P. C. Jones, Y. Zhang, and S. R. Madden, "Schism: a workloaddriven approach to database replication and partitioning," 2010.

[100] A. Pavlo, C. Curino, and S. Zdonik, "Skew-aware automatic database partitioning in shared-nothing, parallel oltp systems," in Proceedings of the 2012 ACM SIGMOD International Conference on Management of Data, pp. 61-72, 2012.

[101] A. L. Tatarowicz, C. Curino, E. P. Jones, and S. Madden, "Lookup tables: Finegrained partitioning for distributed databases," in 2012 IEEE 28th International Conference on Data Engineering, pp. 102-113, IEEE, 2012.

[102] A. Quamar, K. A. Kumar, and A. Deshpande, "Sword: scalable workload-aware data placement for transactional workloads," in Proceedings of the 16th international conference on extending database technology, pp. 430-441, 2013.

[103] Z. Khayyat, K. Awara, A. Alonazi, H. Jamjoom, D. Williams, and P. Kalnis, "Mizan: A system for dynamic load balancing in large-scale graph processing," in Proceedings of the 8th ACM European Conference on Computer Systems, 2013.

[104] C. Martella, D. Logothetis, A. Loukas, and G. Siganos, "Spinner: Scalable graph partitioning in the cloud," in 33rd IEEE ICDE International Conference on Data Engineering, 2017.

[105] F. Rahimian, A. H. Payberah, S. Girdzijauskas, M. Jelasity, and S. Haridi, "A distributed algorithm for large-scale graph partitioning," Proceedings of the ACM TAAS Transactions on Autonomous and Adaptive Systems, vol. 10, no. 2, 2015 .

[106] Z. Shang and J. X. Yu, "Catch the wind: Graph workload balancing on cloud," in 29th IEEE ICDE International Conference on Data Engineering, 2013.

[107] L. Vaquero, F. Cuadrado, D. Logothetis, and C. Martella, "xDGP: A dynamic graph processing system with adaptive partitioning," arXiv preprint arXiv:1309.1049, 2013. 
[108] L. M. Vaquero, F. Cuadrado, D. Logothetis, and C. Martella, "Adaptive partitioning for large-scale dynamic graphs," in 34th IEEE International Conference on Distributed Computing Systems, 2014.

[109] N. Xu, L. Chen, and B. Cui, "LogGP: A log-based dynamic graph partitioning method," Proceedings of the VLDB Endowment, vol. 7, no. 14, 2014.

[110] A. Zheng, A. Labrinidis, and P. K. Chrysanthis, "Planar: Parallel lightweight architecture-aware adaptive graph repartitioning," in 32nd IEEE ICDE International Conference on Data Engineering, 2016.

[111] A. Zheng, A. Labrinidis, and C. Faloutsos, "Skew-Resistant Graph Partitioning," in 33rd IEEE ICDE International Conference on Data Engineering, 2017.

[112] J. Mondal and A. Deshpande, "Managing large dynamic graphs efficiently," in International Conference on Management of Data, 2012.

[113] J. M. Pujol, V. Erramilli, G. Siganos, X. Yang, N. Laoutaris, P. Chhabra, and P. Rodriguez, "The little engine (s) that could: scaling online social networks," Proceedings of the ACM SIGCOMM Computer Communication Review, vol. 41, no. 4, pp. 375-386, 2011.

[114] R. Angles and C. Gutierrez, "An introduction to graph data management," in Graph Data Management, pp. 1-32, Springer, 2018.

[115] S. Heidari, Y. Simmhan, R. N. Calheiros, and R. Buyya, "Scalable graph processing frameworks: A taxonomy and open challenges," ACM Computing Surveys, vol. 51, no. 3, pp. 1-53, 2018.

[116] A. Buluç, H. Meyerhenke, I. Safro, P. Sanders, and C. Schulz, "Recent advances in graph partitioning," in Algorithm Engineering, pp. 117-158, Springer, 2016.

[117] A. Pacaci and M. T. Özsu, "Experimental analysis of streaming algorithms for graph partitioning," in International Conference on Management of Data, pp. 1375-1392, ACM, 2019.

[118] P. Peng, L. Zou, L. Chen, and D. Zhao, "Query workload-based rdf graph fragmentation and allocation," EDBT, 2016.

[119] J. Huang, D. J. Abadi, and K. Ren, "Scalable sparql querying of large rdf graphs," Proceedings of the VLDB Endowment, vol. 4, no. 11, pp. 1123-1134, 2011. 
[120] R. Angles, M. Arenas, P. Barcelo, A. Hogan, J. Reutter, and D. Vrgoc, "Foundations of modern graph query languages," arXiv preprint arXiv:1610.06264, 2016.

[121] R. Angles, "A comparison of current graph database models," in 28th International Conference on Data Engineering Workshops (ICDEW), pp. 171-177, IEEE, 2012.

[122] K. Zeng, J. Yang, H. Wang, B. Shao, and Z. Wang, "A distributed graph engine for web scale RDF data," in Proceedings of the VLDB Endowment, vol. 6, pp. 265-276, 2013.

[123] J. Shi, Y. Yao, R. Chen, H. Chen, and F. Li, "Fast and Concurrent RDF Queries with RDMA-Based Distributed Graph Exploration," in $O S D I$, vol. 16, pp. 317$332,2016$.

[124] I. Stanton and G. Kliot, "Streaming graph partitioning for large distributed graphs," in Proceedings of the 18th ACM SIGKDD International Conference on Knowledge Discovery and Data Mining, 2012.

[125] G. Aluç, O. Hartig, M. T. Özsu, and K. Daudjee, "Diversified stress testing of rdf data management systems," in International Semantic Web Conference, pp. 197-212, Springer, 2014.

[126] SWAT-Projects, "The Lehigh University Benchmark (LUBM)," [Online].

[127] J. Lehmann, R. Isele, M. Jakob, A. Jentzsch, D. Kontokostas, P. N. Mendes, S. Hellmann, M. Morsey, P. Van Kleef, S. Auer, et al., "DBpedia-a large-scale, multilingual knowledge base extracted from wikipedia," Semantic web, vol. 6, no. 2, pp. 167-195, 2015.

[128] T. Stegemann and J. Ziegler, "Pattern-based analysis of sparql queries from the lsq dataset.," in International Semantic Web Conference (Posters, Demos \&6 Industry Tracks), pp. 1-4, 2017. 